\title{
Weighted Bergman Kernels and Mathematical Physics
}

\author{
Elisabetta Barletta ${ }^{1}$, Sorin Dragomir ${ }^{1, *}$ and Francesco Esposito ${ }^{2}$ \\ 1 Dipartimento di Matematica, Università degli Studi della Basilicata, Informatica, ed Economia, \\ Via dell'Ateneo Lucano 10, 85100 Potenza, Italy; elisabetta.barletta@unibas.it \\ 2 Dipartimento di Matematica e Fisica Ennio De Giorgi, Università del Salento, 73100 Lecce, Italy; \\ francesco.esposito@unisalento.it \\ * Correspondence: sorin.dragomir@unibas.it
}

Received: 20 December 2019; Accepted: 15 April 2020; Published: 29 April 2020

check for updates

\begin{abstract}
We review several results in the theory of weighted Bergman kernels. Weighted Bergman kernels generalize ordinary Bergman kernels of domains $\Omega \subset \mathbb{C}^{n}$ but also appear locally in the attempt to quantize classical states of mechanical systems whose classical phase space is a complex manifold, and turn out to be an efficient computational tool that is useful for the calculation of transition probability amplitudes from a classical state (identified to a coherent state) to another. We review the weighted version (for weights of the form $\gamma=|\varphi|^{m}$ on strictly pseudoconvex domains $\left.\Omega=\{\varphi<0\} \subset \mathbb{C}^{n}\right)$ of Fefferman's asymptotic expansion of the Bergman kernel and discuss its possible extensions (to more general classes of weights) and implications, e.g., such as related to the construction and use of Fefferman's metric (a Lorentzian metric on $\partial \Omega \times S^{1}$ ). Several open problems are indicated throughout the survey.
\end{abstract}

Keywords: admissible weight; reproducing kernel Hilbert space; weighted Bergman kernel; weighted Ramadanov theorem; suspended domain; quantum phase space; Bergman metric; Segal-Bargmann-Fock space; Segal-Bargmann transform; canonical Hermitian connection; Liouville measure; transition probability amplitude; complex orbifold; epsilon function; Djrbashian kernel; Forelli-Rudin-Ligocka-Peloso asymptotic expansion formula; Fefferman metric; Cartan connection

\section{Introduction}

The present paper is a survey of known results on the mathematical analysis of weighted Bergman kernels and their applications to mathematical physics, such as the theory of quantization of states of mechanical systems, and also back to complex analysis where some of the matters regarding weighted Bergman kernels arise. There is an ample mathematics literature devoted to both sides-as suggested by the title of the survey - of the subject matter, and only a small part of that was reported on: Authors' choice was based on their personal taste and area of expertise, on the limits of their scientific understanding, and of course on the huge amount of material that was to be reviewed sine qua non. The second named author learned about weighted Bergman kernels many years ago from Z. Pasternak-Winiarski's work [1], followed by [2] where the author introduces the reader to A. Odzijewicz's fundamental work [3], and implicitly to the cultural universe of works authored by Anatol Odzijewicz and his collaborators (cf. M. Horowski and A. Odzijewicz, [4,5], G. Jakimowicz and A. Odzijewicz, [6], W. Lisiecki and A. Odzijewicz, [7], A. Odzijewicz, [3,8-10], A. Odzijewicz and A. Ryzko, [11], as well as A. Odzijewicz and M. Swietochowski, [12]). Over the years both the first and second author were interested in the boundary behavior of the (ordinary, unweighted) Bergman kernel, starting from the pioneering work by N. Kerzman (cf. [13]) and L. Hörmander (cf. [14]) and from the differential geometric consequences (e.g., the occurrence of the Fefferman metric-a Lorentzian metric on $\partial \Omega \times S^{1}$, associated to any smoothly bounded strictly pseudoconvex domain $\left.\Omega \subset \mathbb{C}^{n}\right)$ of C. Fefferman's work [15]. The construction of the Fefferman metric relies on results in C. 
Fefferman's work [16] (i.e., on the asymptotic expansion of the Bergman kernel there), and any attempt to discover similar differential geometric objects within the realm of weighted Bergman kernels should be preceded by producing an analog to said asymptotic expansion for a $\gamma$-Bergman kernel $K_{\gamma}(z, \zeta)$ corresponding to an admissible weight $\gamma \in W(\Omega)$. Such an analog to said asymptotic expansion was obtained by M.M. Peloso (cf. $[17,18]$ ) yet confined to the class of weights which are integer powers of the defining function-that is, $|\varphi|^{m} \in A W(\Omega)$, where $m \in \mathbb{Z}_{+}$and $\Omega=\{\varphi<0\}$. M.M. Peloso claims his asymptotic expansion formula for $K_{|\varphi|^{m}}(z, \zeta)$ is implicit in the work by E. Ligocka (cf. [19]), who in turn relies on older ideas by F. Forelli and W. Rudin (cf. [20]). To settle matters regarding credit for authorship we refer to the mentioned result as the Forelli-Rudin-Ligocka-Peloso asymptotic expansion formula. The problem of extending the asymptotic expansion to the (more general class of) weights of the form $\gamma=|\varphi|^{\alpha} \exp (g), \alpha>-1$, was taken up by E. Barletta et al. (cf. [21]) when $\alpha=m \in \mathbb{Z}_{+}$and by M. Englis (cf. [22]) for arbitrary $\alpha>-1$. Although of course less general, the case of $K_{|\varphi|^{m}}(z, \zeta)$ appears as particularly appealing, for $|\varphi|^{m}$-Bergman kernels can be related to ordinary Bergman kernels by using the "suspended domains" of F. Forelli and W. Rudin (cf. op. cit.). We remind the reader that the first use of the asymptotic expansion for the Bergman kernel was in the proof of C. Fefferman's celebrated result (cf. [16]) that biholomorphisms $F: \Omega_{1} \rightarrow \Omega_{2}$ of smoothly bounded strictly pseudoconvex domains $\Omega_{j} \subset \mathbb{C}^{n}, j \in\{1,2\}$, extend smoothly up to the boundary (and give CR isomorphisms $f: \partial \Omega_{1} \rightarrow \partial \Omega_{2}$ of the boundaries). On the other hand, I. Naruki (cf. [23]) gave an alternative-more geometric, as we shall emphasize shortly-proof to what is now called Fefferman's theorem, prior to that of Fefferman. There were some gaps in the original version of Naruki's proof, and only a short version was published in the end (as the paper [23]). It reduces Fefferman's theorem to a result about extensions of Cartan connections and makes a creative use of suspended domains. As manifest in [15], C. Fefferman learned about suspended domains from [23], and that was the key ingredient in the construction of the Lorentzian metric that bears his name. There is a fascinating formal resemblance between Naruki's G-admissible metrics and B.G. Schmidt's bundle boundary constructions (cf. [24]) suggesting a connection that ought to be discovered among the mathematical analysis of weighted Bergman kernels and the physics of space-time singularities (cf. e.g., C.J.S. Clarke [25]). This is but one of the many open questions raised through the present survey.

The exposition is organized as follows. The main mathematical analysis results about weighted Bergman kernels are presented in Sections 1-3. Authors' choice of mathematical physics results as related to the theory of weighted Bergman kernels occupies Section 4 and relies mainly on the scientific creation of K. Gawdezki (cf. [26]), B. Kostant (cf. [27]), and of course A. Odzijewicz (cf. [3]). Section 5 is devoted to the discussion of some of Authors' own work (cf. [28]). The Forelli-Rudin-Ligocka-Peloso expansion is discussed in Section 6 which also emphasizes the authors' work [21] (itself relying on the treatment in [2] of weighted Bergman kernels as functions $K: A W(\Omega) \rightarrow H A(\Omega)$ on the Banach manifold of admissible weights) vis-a-vis to that by M. Englis (cf. [22]), although no actual parallel is drawn among the two. The computability of weighted and unweighted Bergman kernels is addressed in Section 7. Naruki's (Cartan-connection-based) proof of Fefferman's theorem is given in Section 8.

\section{Admissible Weights and Reproducing Kernels}

Let $\Omega \subset \mathbb{C}^{n}$ be an open set. Let $W(\Omega)$ denote the set of all Lebesgue measurable functions $\gamma: \Omega \rightarrow(0,+\infty)$. An element $\gamma \in W(\Omega)$ is a weight on $\Omega$. Two weights coinciding a.e. in $\Omega$ are identified. Let $L^{2}(\Omega, \gamma)$ be the space of all Lebesgue measurable functions $f: \Omega \rightarrow \mathbb{C}$ such that

$$
\int_{\Omega}|f(\zeta)|^{2} \gamma(\zeta) d \mu(\zeta)<\infty
$$


where $d \mu$ is the Lebesgue measure on $\mathbb{R}^{2 n}$. Then $L^{2}(\Omega, \gamma)$ is a separable Hilbert space with the $L^{2}$ inner product

$$
(f, g)_{\gamma}=\int_{\Omega} f(\zeta) \overline{g(\zeta)} \gamma(\zeta) d \mu(\zeta)
$$

We also set $\|f\|_{\gamma}=(f, f)_{\gamma}^{1 / 2}$. The map $f \longmapsto \sqrt{\gamma} f$ is an isometry of $L^{2}(\Omega, \gamma)$ onto the ordinary Lebesgue space $L^{2}(\Omega)$. Let $L^{2} H(\Omega, \gamma)=L^{2}(\Omega, \gamma) \cap \mathcal{O}(\Omega)$ be the space of all holomorphic functions in $L^{2}(\Omega, \gamma)$. Then $L^{2} H(\Omega, \gamma)$ is referred to as the $\gamma$-Bergman space over $\Omega$.

A weight $\gamma \in W(\Omega)$ is admissible if (i) the evaluation functional $\delta_{z}: L^{2} H(\Omega) \rightarrow \mathbb{C}, \delta_{z}(f)=f(z)$, is continuous for any $z \in \Omega$, and ii) $L^{2} H(\Omega, \gamma)$ is a closed subspace of $L^{2}(\Omega, \gamma)$. Let $A W(\Omega)$ denote the set of admissible weights on $\Omega$. As we shall see later on in this paper, $W(\Omega)$ may be organized as an infinite dimensional Banach manifold modelled on $L^{\infty}(\Omega)$ and then $A W(\Omega)$ is an open subset in $W(\Omega)$.

If $\gamma \in A W(\Omega)$ then (by axiom (ii) above) $L^{2} H(\Omega, \gamma)$ is a Hilbert space, with the inner product (1). On the other hand for every $z \in \Omega$ the evaluation functional $\delta_{z}$ is continuous (by axiom (i) above) hence the Riesz representation theorem applies, so that there is $k_{z, \gamma} \in L^{2} H(\Omega, \gamma)$ such that $\delta_{z}(f)=$ $\left(f, k_{z, \gamma}\right)_{\gamma}$ or

$$
f(z)=\int_{\Omega} f(\zeta) \overline{k_{z, \gamma}(\zeta)} \gamma(\zeta) d \mu(\zeta)
$$

for any $f \in L^{2} H(\Omega)$. The function

$$
K_{\gamma}: \Omega \times \Omega \rightarrow \mathbb{C}, \quad K_{\gamma}(z, \zeta)=\overline{k_{z, \gamma}(\zeta)}, \quad z, \zeta \in \Omega,
$$

is the $\gamma$-Bergman kernel of $\Omega$. Formula (2) becomes

$$
f(z)=\int_{\Omega} K_{\gamma}(z, \zeta) f(\zeta) \gamma(\zeta) d \mu(\zeta)
$$

for any $f \in L^{2} H(\Omega)$ and any $z \in \Omega$. As an immediate consequence (by applying (3) for $f \equiv 1$ )

$$
\int_{\Omega} K_{\gamma}(\zeta, \zeta) \gamma(\zeta) d \mu(\zeta)=1
$$

If $\gamma(\zeta) \equiv 1$, then $K(z, \zeta)=K_{1}(z, \zeta)$ is the ordinary Bergman kernel of $\Omega$, as discovered by S. Bergman [29].

Let $E$ be a set and let $\mathcal{F}(E)$ be the set of all complex-valued functions $f: E \rightarrow \mathbb{C}$. A complex Hilbert space $H$ is a reproducing kernel Hilbert space (a RKH space) if $H \subset \mathcal{F}(E)$ for some $E \neq \varnothing$ and for every $x \in E$ the evaluation functional $\delta_{x}: E \rightarrow \mathbb{C}, \delta_{x}(f)=f(x)$, is continuous. Once again, by the Riesz representation theorem, for every $x \in E$ there is a unique $k_{x} \in H$ such that $\delta_{x}(f)=\left(f, k_{x}\right)_{H}$ for any $f \in H$, and the function $k: E \times E \rightarrow \mathbb{C}, k(x, y)=\overline{k_{x}(y)}$, is the reproducing kernel of $H$. Hence $L^{2} H(\Omega, \gamma)$ is a RKH space and $K_{\gamma}$ is its reproducing kernel. See N. Aronszajn [30] for a general theory of RKH spaces. However, the notion of a reproducing kernel is much older and appears to have been first introduced by the famous Polish mathematician S. Zaremba in connection with his work (cf. [31]) on boundary value problems for harmonic and biharmonic functions.

By a result of Z. Pasternak-Winiarski (cf. [1]) weighted Bergman kernels $K_{\gamma}$ enjoy most of the properties of ordinary Bergman kernels. Let $\gamma \in A W(\Omega)$. Then,

(i) For any complete orthonormal system $\left\{\phi_{v}\right\}_{v \geq 0} \subset L^{2} H(\Omega, \gamma)$ the series $\sum_{v \geq 0} \phi_{v}(z) \overline{\phi_{v}(\zeta)}$ converges uniformly on any compact subset of $\Omega \times \Omega$ and its sum is

$$
\sum_{v=0}^{\infty} \phi_{v}(z) \overline{\phi_{v}(\zeta)}=K_{\gamma}(z, \zeta)
$$


for any $z, \zeta \in \Omega$.

(ii) For any $z, \zeta \in \Omega$

$$
K_{\gamma}(\zeta, z)=\overline{K_{\gamma}(z, \zeta)}
$$

(iii) $K_{\gamma}(z, \zeta)$ is holomorphic in $z$ and anti-holomorphic in $\zeta$.

(iv) $K_{\gamma}$ is real analytic.

(v) If $P_{\gamma}: L^{2}(\Omega, \gamma) \rightarrow L^{2} H(\Omega, \gamma)$ is the $L^{2}$ orthogonal projection then

$$
\left(P_{\gamma} f\right)(z)=\int_{\Omega} K_{\gamma}(z, \zeta) f(\zeta) d \mu(\zeta)
$$

for any $f \in L^{2}(\Omega, \gamma)$ and any $z \in \Omega$.

Cf. Theorem 2.1 in [1], p. 3. Given a weight $\gamma \in W(\Omega)$, the following statements are equivalent (providing several characterizations of admissibility)

(a) $\gamma \in A W(\Omega)$.

(b) For every compact subset $A \subset \Omega$ there is a constant $C_{A}>0$ such that

$$
|f(z)| \leq C_{A}\|f\|_{\gamma}
$$

for any $f \in L^{2} H(\Omega)$ and any $z \in A$.

(c) For every $z \in \Omega$ there is a compact subset $B \subset \Omega$ such that $z \in B$, and for any $w \in \partial B$ there is an open neighborhood $V \subset \Omega$ of $w$ and a constant $C>0$ such that $|f(\zeta)| \leq C\|f\|_{\gamma}$ for any $\zeta \in V$ and any $f \in L^{2} H(\Omega, \gamma)$. (d) For every $z \in \Omega$ there is an open neighborhood $V \subset \Omega$ of $z$ and a constant $C>0$ such that $|f(\zeta)| \leq C\|f\|_{\gamma}$ for any $\zeta \in V$ and any $f \in L^{2} H(\Omega, \gamma)$.

Cf. Theorem 2.2 in [1], p. 4. Following [1] we give a few sufficient conditions for the admissibility of a weight $\gamma \in W(\Omega)$, which are in mathematical practice easier to check than any of the conditions (b)-(d) above. Precisely, let $\gamma \in W(\Omega)$ and let us assume that there is an open set $U \subset \Omega$ and a positive number $a>0$ such that $\gamma^{-a} \in L^{1}(U)$. Then, for every $z \in U$ there is an open neighborhood $V \subset \Omega$ of $z$ and a constant $C>0$ such that $|f(\zeta)| \leq C\|f\|_{\gamma}$ for all $\zeta \in V$ and all $f \in L^{2} H(\Omega)$. Cf. Theorem 3.1 in [1], p. 5.

The proof relies on the relationship between holomorphic functions and subharmonic functions, and is quantitatively relevant enough - at least for the more physics-oriented reader- to be reproduced in this survey. Toward this end, for every $z \in U$ let $r>0$ such that $B_{2 r}(z) \subset U$. Next let us set $V=B_{r}(z)$ so that $\overline{B_{r}(\zeta)} \subset U$ for any $\zeta \in V$. Indeed, for every $w \in \overline{B_{r}(\zeta)}$

$$
|w-z| \leq|w-\zeta|+|\zeta-z|<2 r \Longrightarrow w \in B_{2 r}(z) \subset U .
$$

By a classical result in complex analysis (cf. e.g., Corollary 2.1.15 in [32], p. 75) if $f \in \mathcal{O}(\Omega)$ then $|f|^{P}$ is subharmonic for every $P>0$. Let $p=(1+a) / a>1$ and $P=2 / p$. Then, for every $f \in L^{2} H(\Omega)$ and every $\zeta \in V$

$$
\begin{gathered}
|f(\zeta)|^{2 / p} \leq \frac{1}{\operatorname{Vol}\left[B_{r}(\zeta)\right]} \int_{B_{r}(\zeta)}|f(w)|^{2 / p} d \mu(w)= \\
=\frac{1}{\operatorname{Vol}\left[B_{r}(\zeta)\right]} \int_{B_{r}(\zeta)}|f(w)|^{2 / p} \gamma(w)^{1 / p} \gamma(w)^{-1 / p} d \mu(w) \leq
\end{gathered}
$$

(by Hölder's inequality with $1 / p+1 / q=1$, and hence with $q=1+a$ )

$$
\leq \frac{1}{\operatorname{Vol}\left[B_{r}(\zeta)\right]}\left(\int_{B_{r}(\zeta)}|f|^{2} \gamma d \mu\right)^{1 / p}\left(\int_{B_{r}(\zeta)} \gamma^{-q / p} d \mu\right)^{1 / q}
$$


hence

$$
\begin{gathered}
|f(\zeta)| \leq \frac{1}{\operatorname{Vol}\left[B_{r}(\zeta)\right]^{p / 2}}\left(\int_{B_{r}(\zeta)}|f|^{2} \gamma d \mu\right)^{1 / 2}\left(\int_{B_{r}(\zeta)} \gamma^{-q / p} d \mu\right)^{p /(2 q)} \leq \\
\leq C\|f\|_{\gamma^{\prime}} \\
C=\frac{1}{\operatorname{Vol}\left[B_{r}(\zeta)\right]^{(1+a) /(2 a)}}\left(\int_{U} \gamma^{-a} d \mu\right)^{1 /(2 a)} .
\end{gathered}
$$

Q.e.d.

Theorem 1 (Cf. [1], p. 6). Let $\gamma \in W(\Omega)$. Let us assume that for every $\zeta \in \Omega$ there is a compact set $A \subset \Omega$ such that $\zeta \in A$ and for every $w \in \partial A$ there is an open neighbourhood $U_{w} \subset \Omega$ of $w$ and a number $a_{w}>0$ such that $\gamma^{-a_{w}} \in L^{1}\left(U_{w}\right)$. Then $\gamma \in A W(\Omega)$. In particular, if $\gamma^{-a} \in L_{\mathrm{loc}}^{1}(\Omega)$ for some $a>0$, then $\gamma \in A W(\Omega)$.

Example 1 (Admissible weights on $\mathbb{B}^{1}$ ). Let $\Omega=\mathbb{B}^{1}=\{z \in \mathbb{C}:|z|<1\}$ be the unit disc and let us set

$$
\begin{gathered}
f_{t}(z)=|\operatorname{Im}(z)|^{t}, \quad t \in(0,+\infty), \\
g(z)=|\operatorname{Im}(z)|^{1 /[1-|z|]}, \quad h(z)= \begin{cases}\exp \left[|z|^{-1 / 2}\right] & \text { for } z \neq 0, \\
0 & \text { for } z=0,\end{cases}
\end{gathered}
$$

for every $z \in \mathbb{B}^{1}$. Then (cf. [1], p. 7)

$$
f_{t}, g, h \in A W\left(\mathbb{B}^{1}\right), \quad t \in(0,+\infty) .
$$

Here one may decide on admissibility by using Corollary 1.

For further use, let us observe that given an admissible weight $\gamma \in A W(\Omega)$ and the corresponding reproducing kernel $K_{\gamma}$, for any compact set $A \subset \Omega$ there is a constant $C_{A}>0$ such that for any $\zeta \in A$

$$
\begin{gathered}
\left\|\delta_{\zeta}\right\|_{\gamma} \leq C_{A}, \\
\left\|K_{\gamma}(\zeta, \cdot)\right\|_{\gamma} \leq C_{A} .
\end{gathered}
$$

The norm of $\delta_{\zeta}$ in (5) is meant as an element of the topological dual $L^{2} H(\Omega)^{*}$, that is,

$$
\left\|\delta_{\zeta}\right\|_{\gamma}=\sup \left\{\left|\delta_{\zeta}(f)\right|: f \in L^{2} H(\Omega, \gamma),\|f\|_{\gamma}=1\right\}
$$

and for any compact set $A \subset \Omega$ with $\zeta \in A$ there is $C_{A}>0$ such that $\left|\delta_{\zeta}(f)\right|=|f(\zeta)| \leq C_{A}\|f\|_{\gamma}$ for any $f \in L^{2} H(\Omega, \gamma)$, thus yielding (5). As to (6), let us recall that $K_{\gamma}(\zeta, z)=\overline{k_{\zeta, \gamma}(z)}$ for some $k_{\zeta, \gamma} \in L^{2} H(\Omega, \gamma)$, hence (by estimate (4) for $f=k_{\zeta, \gamma}$ )

$$
\left|k_{\zeta, \gamma}(z)\right| \leq C_{A}\left\|k_{\zeta, \gamma}\right\|_{\gamma}
$$

for any $z \in A$. On the other hand

$$
\begin{aligned}
& \left\|k_{\zeta, \gamma}\right\|_{\gamma}^{2}=\int_{\Omega}\left|k_{\zeta, \gamma}(w)\right|^{2} \gamma(w) d \mu(w)= \\
& =\int_{\Omega} K_{\gamma}(\zeta, w) \overline{K_{\gamma}(\zeta, w)} \gamma(w) d \mu(w)= \\
& =\int_{\Omega} K_{\gamma}(\zeta, w) K_{\gamma}(w, \zeta) \gamma(w) d \mu(w)=
\end{aligned}
$$


(by (3) for $f=K_{\gamma}(\cdot, \zeta)$ )

$$
=K_{\gamma}(\zeta, \zeta)
$$

hence (7) yields

$$
\left|K_{\gamma}(\zeta, z)\right| \leq C_{A} K_{\gamma}(\zeta, \zeta)^{1 / 2} .
$$

Finally we may estimate $\left\|K_{\gamma}(\zeta, \cdot)\right\|_{\gamma}$ as follows:

$$
\left\|K_{\gamma}(\zeta, \cdot)\right\|_{\gamma}^{2}=\int_{\Omega}\left|K_{\gamma}(\zeta, z)\right|^{2} \gamma(z) d \mu(z) \leq
$$

(by interchanging $\zeta$ and $z$ in (8))

$$
\leq C_{A}^{2} \int_{\Omega} K_{\gamma}(z, z) \gamma(z) d \mu(z)=C_{A}^{2} .
$$

Q.e.d.

In his pioneering paper [33], G. Cimmino studied the Dirichlet problem for the ordinary Laplacian on domains in $\mathbb{R}^{2}$ with $L^{2}$ boundary data. We restate Cimmino's approach (accredited by him to $R$. Caccioppoli [34]) in a slightly generalized form, on domains $\Omega \subset \mathbb{C}^{n}$.

Example 2 (Cimmino's admissible weights). Let $\gamma \in W(\Omega)$ and $f \in L^{2}(\partial \Omega)$, and let $\mathcal{F}$ be a foliation by real hypersurfaces of $\mathbb{C}^{n}$, of a one sided neighbourhood $V \subset \Omega$ of the boundary $\partial \Omega$. Moreover, let $\left\{\Phi_{L}\right\}_{L \in V / \mathcal{F}}$ be a family of $C^{\infty}$ diffeomorphisms $\Phi_{L}: \partial \Omega \backslash F \rightarrow L$, for some subset $F \subset \partial \Omega$ of "surface" measure zero. Given a function $u: \Omega \rightarrow \mathbb{C}$ we say that $u=f$ on $\partial \Omega$ if

$$
\lim _{\epsilon \rightarrow 0^{+}} \int_{\partial \Omega}\left|u \circ \Phi_{L_{\epsilon}}-f\right|^{2}\left(\gamma \circ \Phi_{L_{\epsilon}}\right) d \sigma=0
$$

for any generalized sequence of leaves $\left\{L_{\epsilon}\right\}_{\epsilon>0} \subset V / \mathcal{F}$ tending to $\partial \Omega$ in the Gromov-Hausdorff distance as $\epsilon \rightarrow 0^{+}$.

As emphasized by G. Cimmino (cf. op. cit., p. 10, or [35], p. 266) the choice of data $\left(\gamma, f, \mathcal{F},\left\{\Phi_{L}\right\}_{L \in V / \mathcal{F}}\right)$ is eventually responsible for the loss of uniqueness in the Dirichlet problem $\Delta u=0$ in $\Omega, u=f$ on $\partial \Omega$. For instance, let $\Omega=\mathbb{B}^{n}$ be the unit ball in $\mathbb{C}^{n}$ (with $n=1$ in [33]) and $\zeta_{0} \in \partial \mathbb{B}^{n}$ and let us set

$$
\gamma(\zeta)=\left|\zeta-\zeta_{0}\right|^{2}, \quad \zeta \in \Omega .
$$

If $A \subset \Omega$ is a compact subset and $d=\operatorname{dist}\left(\zeta_{0}, A\right)>0$ then $\int_{A} \gamma^{-1} d \mu \leq d^{2} \mu(A)<\infty$, hence $\gamma^{-1} \in$ $L_{\text {loc }}^{1}(\Omega)$ so that (by Corollary 1 with $\left.a=1\right) \gamma \in A W\left(\mathbb{B}^{n}\right)$. Let $\mathcal{F}$ be the foliation of $V=\mathbb{B}^{n} \backslash\{0\}$ whose leaf space is

$$
V / \mathcal{F}=\left\{S^{2 n-1}(0,1-t): 0<t<1\right\} .
$$

Here $S^{2 n-1}(z, r)=\partial B_{r}(z)$ is the sphere of radius $r>0$ and center $z \in \mathbb{C}^{n}$. If $L_{\epsilon}=S^{2 n-1}(0,1-\epsilon)$, $0<\epsilon<1$, then

$$
\Phi_{\epsilon}: \partial \mathbb{B}^{n} \rightarrow L_{\epsilon}, \quad \Phi_{\epsilon}(\zeta)=(1-\epsilon) \zeta,
$$

is a $C^{\infty}$ diffeomorphism. Let us consider the function

$$
u(\zeta)=\frac{1-|\zeta|^{2}}{\left|\zeta-\zeta_{0}\right|^{2}}, \quad \zeta \in \mathbb{B}^{n}
$$

Then

$$
\lim _{\epsilon \rightarrow 0^{+}} \int_{\partial \mathbb{B}^{n}}\left|u\left(\Phi_{\epsilon}(z)\right)\right|^{2} \gamma\left(\Phi_{\epsilon}(z)\right) d \sigma(z)=0
$$


so $u=0$ on $\partial \mathbb{B}^{n}$ with respect to the data $\left(\gamma, \mathcal{F},\left\{\Phi_{\epsilon}\right\}_{\epsilon>0}\right)$ chosen above. On the other hand, if $n=1$ and $\zeta_{0}=1$ then $u=\operatorname{Re}(h)$ with $h(\zeta)=(1+\zeta) /(1-\zeta)$. Here $h$ is holomorphic, hence $u$ is a nonzero harmonic function on $\mathbb{B}^{1}$ having zero boundary data in the $L^{2}$ sense adopted by $C$. Cimmino, accounting for non-uniqueness in the Dirichlet problem. We emphasize, together with C. Cimmino (cf. op. cit.), that non-uniqueness is produced by the vanishing of the weight on a portion of the boundary (a point, in the present example). To be entirely fair to the reader, the phenomenon is not governed by the weight alone. Indeed, let $\gamma \equiv 1$ and let $\mathcal{F}$ be the foliation of $\mathbb{B}^{n}$ whose leaf space is

$$
\mathbb{B}^{n} / \mathcal{F}=\left\{L_{t}: 0<t<1\right\}, \quad L_{t}=S^{2 n-1}\left(t \zeta_{0}, 1-t\right),
$$

and let $\Phi_{t}: \partial \mathbb{B}^{n} \backslash\left\{\zeta_{0}\right\} \rightarrow L_{t}$ be defined by

$$
\begin{gathered}
\left\{\Phi_{t}(\zeta)\right\}=\ell_{\zeta} \cap L_{t}, \quad \zeta \in \partial \mathbb{B}^{n} \backslash\left\{\zeta_{0}\right\}, \\
\ell_{\zeta}=\left\{(1-s) \zeta_{0}+s \zeta: 0<s<1\right\} .
\end{gathered}
$$

The intersection point $\Phi_{t}(\zeta)$ corresponds to the value of the parameter $s=(1-t) /\left|\zeta-\zeta_{0}\right|$. Once again, $\lim _{t \rightarrow 0^{+}} \int_{\partial \mathbb{B}^{n}}\left|u \circ \Phi_{t}\right|^{2} d \sigma=0$ (and if $n=1$ and $\zeta_{0}=1$ then $u$ is a nonzero harmonic function on $\mathbb{B}^{1}$ ).

Next we give an example due to F. Forelli and W. Rudin (cf. [20]). The fact that their construction fits into the the theory of weighted Bergman kernels was observed by E. Ligocka (cf. [19]).

Example 3 (Forelli and Rudin's admissible weight). Let $s=\sigma+i t \in \mathbb{C}$ be a complex number with $\sigma>-1$ and $t \in \mathbb{R}$.

Let us set

$$
\gamma_{s}(\zeta)=\left(1-|\zeta|^{2}\right)^{s}, \quad \zeta \in \mathbb{B}^{n}
$$

Then $\gamma_{s} \in A W\left(\mathbb{B}^{n}\right)$ and the corresponding $\gamma_{s}$-Bergman kernel is

$$
\begin{gathered}
K_{\gamma_{s}}(\zeta, z)=\frac{\left(\begin{array}{c}
n+s \\
n
\end{array}\right)}{(1-\zeta \cdot \bar{z})^{n+1+s}}, \quad \zeta, z \in \mathbb{B}^{n}, \\
\left(\begin{array}{c}
n+s \\
n
\end{array}\right)=\frac{\Gamma(n+s+1)}{\Gamma(n+1) \Gamma(s+1)} .
\end{gathered}
$$

The complex powers in (9) and (10) are understood as the ordinary principal branches. $L^{2} H\left(\mathbb{B}^{n}, \gamma_{s}\right)$ is a RKH space with the inner product

$$
(f, g)_{\gamma_{s}}=\int_{\mathbb{B}^{n}} f(\zeta) \overline{g(\zeta)}\left(1-|\zeta|^{2}\right)^{s} d \mu(\zeta)
$$

We close the section with an example building on the work by M.M. Djrbashian and A.H. Karapetyan (cf. [36]), and due to E. Barletta et al. [28].

Example 4 (Djrbashian kernels). Let $\Omega_{n}=\left\{\zeta \in \mathbb{C}^{n}: \operatorname{Im}\left(\zeta_{1}\right)>\left|\zeta^{\prime}\right|\right\}$ be the Siegel domain. Here we set $\zeta^{\prime}=\left(\zeta_{2}, \cdots, \zeta_{n}\right)$ for every $\zeta=\left(\zeta_{1}, \cdots, \zeta_{n}\right) \in \mathbb{C}^{n}$. For every $\alpha>-1$ let $\gamma_{\alpha} \in W\left(\Omega_{n}\right)$ be the weights given by

$$
\gamma_{\alpha}(\zeta)=\left[\operatorname{Im}\left(\zeta_{1}\right)-\left|\zeta^{\prime}\right|^{2}\right]^{\alpha}, \quad \zeta \in \Omega_{n}
$$


The spaces $L^{2} H\left(\Omega_{n}, \gamma_{\alpha}\right)$ coincide with the function spaces $H_{\alpha}^{2}\left(\Omega_{n}\right)$ introduced in [36]. By a result in [28] $\gamma_{\alpha} \in A W\left(\Omega_{n}\right)$ and the corresponding $\gamma_{\alpha}$-Bergman kernel is

$$
\begin{aligned}
K_{\gamma_{\alpha}}(\zeta, z) & =\frac{2^{n-1+\alpha} c_{n, \alpha}}{\left[i\left(\bar{z}_{1}-\zeta_{1}\right)-2\left\langle\zeta^{\prime}, z^{\prime}\right\rangle\right]^{n+1+\alpha}}, \\
c_{n, \alpha} & =\pi^{-n}(\alpha+1) \cdots(\alpha+n) .
\end{aligned}
$$

3. Mathematical Analysis of the Function $\gamma \mapsto K_{\gamma}$

\subsection{Banach Manifold of Weights}

Let $H A(\Omega)$ denote the space of all functions $F: \Omega \times \Omega \rightarrow \mathbb{C}$ such that $F$ is holomorphic in the first $n$ variables, and anti-holomorphic in the last $n$-variables. Then $H A(\Omega)$ is a Fréchet space with the locally convex topology determined by the family of semi-norms

$$
\begin{gathered}
\left\{\|\cdot\|_{A}: A \subset \Omega, A \text { compact }\right\}, \\
\|F\|_{A}=\sup _{(\zeta, z) \in A \times A}|F(\zeta, z)|, \quad F \in H A(\Omega) .
\end{gathered}
$$

The regularity properties (continuity, differentiability, analyticity) of the map

$$
\gamma \in A W(\Omega) \longmapsto K_{\gamma} \in H A(\Omega)
$$

were studied by Z. Pasternak-Winiarski, cf. [2]. To make sense of those regularity properties one needs to organize $W(\Omega)$ as a manifold of sorts. We shall also need the following concept of the analyticity of functions $F: U \subset \mathfrak{X} \rightarrow \mathfrak{Y}$, where $\mathfrak{X}, \mathfrak{Y}$ are respectively a normed space, with the norm $\|\cdot\|_{\mathfrak{X}}$, and an arbitrary topological vector space, and $U \subset \mathfrak{X}$ is an open subset. A function $F: U \rightarrow \mathfrak{Y}$ is analytic on $U$ if for any $x \in U$ there is a ball $B \subset \mathfrak{X}$ of center $\zeta$ with $x+B \subset U$, and there is a sequence $\left\{a_{m}\right\}_{m \in \mathbb{N}}$ of continuous multi-linear ( $m$-linear) maps $a_{m}: \mathfrak{X}^{m} \rightarrow \mathfrak{Y}$ such that

$$
F(x+h)=F(x)+\sum_{m=1}^{\infty} a_{m}(h, \cdots, h)
$$

and the series $\sum_{m \geq 1} a_{m}(h, \cdots, h)$ converges uniformly on B. Cf. e.g., [37], p. 14 (where however $\mathfrak{X}=\mathbb{R}^{n}$ and $\mathfrak{Y}=\mathbb{R}^{m}$ ).

Let $L^{\infty}(\Omega)$ be the Banach algebra of all real-valued essentially bounded functions $g: \Omega \rightarrow \mathbb{R}$ with the norm

$$
\begin{gathered}
\|g\|_{\infty}=\operatorname{esssup}_{x \in \Omega}|g(z)|= \\
=\inf \{K>0:|g(z)| \leq K \text { for a.e. } z \in \Omega\}, \quad g \in L^{\infty}(\Omega) .
\end{gathered}
$$

We also set

$$
\begin{gathered}
\operatorname{essinf}_{z \in \Omega} g(z)=\sup \{L \in \mathbb{R}: L \leq g(z) \text { for a.e. } z \in \Omega\}, \\
U(\Omega)=\left\{g \in L^{\infty}(\Omega): \operatorname{ess} \inf _{z \in \Omega} g(z)>0\right\},
\end{gathered}
$$

so that $U(\Omega)$ is an open subset of $L^{\infty}(\Omega)$. For every $\gamma \in W(\Omega)$ let us consider the map

$$
\begin{gathered}
\Phi_{\gamma}: U(\Omega) \rightarrow W(\Omega), \\
{\left[\Phi_{\gamma}(g)\right](z)=g(z) \gamma(z), \quad g \in U(\Omega), \quad z \in \Omega .}
\end{gathered}
$$

Let us set $U(\Omega, \gamma)=\Phi_{\gamma}[U(\Omega)]$. The maps $\Phi_{\gamma}$ enjoy the following properties (cf. [2], p. 116):

(i) $\Phi_{\gamma}: U(\Omega) \rightarrow W(\Omega)$ is injective. 
(ii) For every $\varphi \in W(\Omega)$

$$
U(\Omega, \varphi) \cap U(\Omega, \gamma) \neq \varnothing \Longrightarrow U(\Omega, \varphi)=U(\Omega, \gamma)
$$

(iii) There is a topology $\tau$ on $W(\Omega)$ such that the family $B=\left\{\Phi_{\gamma}(X): \gamma \in W(\Omega), \quad X \subset U(\Omega), \quad X\right.$ open $\}$ is a base for $\tau$.

(iv) Let $\Phi_{\gamma}^{-1}: U(\Omega, \gamma) \rightarrow U(\Omega)$ be the inverse of $\Phi_{\gamma}: U(\Omega) \rightarrow U(\Omega, \gamma)$. Then $\left\{\Phi_{\gamma}^{-1}: \gamma \in W(\Omega)\right\}$ is an analytic atlas on $(W(\Omega), \tau)$, organizing it as a Banach manifold.

(v) If $\varphi_{1}, \varphi_{2} \in U(\Omega, \gamma)$ then $L^{2}\left(\Omega, \varphi_{1}\right)$ and $L^{2}\left(\Omega, \varphi_{2}\right)$ coincide as vector spaces and the norms $\|\cdot\|_{\varphi_{1}}$ and $\|\cdot\|_{\varphi_{2}}$ are equivalent.

(vi) If $\gamma \in A W(\Omega)$ then $U(\Omega, \gamma) \subset A W(\Omega)$. In particular $A W(\Omega)$ is an open subset of $(W(\Omega)$, $\tau)$.

3.2. Analyticity of the Vector Valued Function $\gamma \longmapsto K_{\gamma}$

Let $\gamma \in A W(\Omega)$ and $g \in U(\Omega)$ and $h_{1}, \cdots, h_{k} \in L^{\infty}(\Omega)$. Let $K_{g \gamma}(\zeta, z)$ be the $g \gamma$-Bergman kernel and let us consider the function

$$
\begin{gathered}
{\left[K_{g, \gamma}^{(k)}\left(h_{1}, \cdots, h_{k}\right)\right](\zeta, z)=} \\
=\int_{\Omega} K_{g \gamma}\left(w_{1}, z\right) h_{1}\left(w_{1}\right) \gamma\left(w_{1}\right) d \mu\left(w_{1}\right) \\
\cdot \int_{\Omega} K_{g \gamma}\left(w_{2}, w_{1}\right) h_{2}\left(w_{2}\right) \gamma\left(w_{2}\right) d \mu\left(w_{2}\right) \cdot \\
\vdots \\
\int_{\Omega} K_{g \gamma}\left(w_{k}, w_{k-1}\right) h_{k}\left(w_{k}\right) K_{g \gamma}\left(\zeta, w_{k}\right) \gamma\left(w_{k}\right) d \mu\left(w_{k}\right)
\end{gathered}
$$

for any $\zeta, z \in \Omega$. Additionally, we set

$$
K_{g, \gamma}^{(k)} h^{(k)}=K_{g, \gamma}^{(k)}\left(h_{1}, \cdots, h_{k}\right), \quad h_{1}=\cdots=h_{k}=h .
$$

By a result of Z. Pasternak-Winiarski (cf. Lemma 5.1 in [2], p. 129-130)

$$
\begin{gathered}
K_{g, \gamma}^{(k)}\left(h_{1}, \cdots, h_{k}\right) \in H A(\Omega), \\
{\left[K_{g, \gamma}^{(k)} h^{(k)}\right](z, \zeta)=\overline{\left[K_{g, \gamma}^{(k)} h^{(k)}\right](\zeta, z)}}
\end{gathered}
$$

for all $\zeta, z \in \Omega$. Clearly

$$
K_{g, \gamma}^{(k)}: L^{\infty}(\Omega)^{k} \rightarrow H A(\Omega)
$$

is a $k$-linear map. Let $A \subset \Omega$ be a compact subset. Again by a result in [2], p. 131, there is a constant $C_{A}>0$ such that

$$
\begin{gathered}
\left|\left[K_{g, \gamma}^{(k)}\left(h_{1}, \cdots, h_{k}\right)\right](\zeta, z)\right| \leq \frac{C_{A}^{2}}{i(g)^{k}}\left\|h_{1}\right\|_{\infty} \cdots\left\|h_{k}\right\|_{\infty}, \\
i(g)=\operatorname{ess}_{\inf _{z \in \Omega} g(z),}
\end{gathered}
$$

that is, $K_{g, \gamma}^{(k)}$ is bounded with respect to the semi-norm $\|\cdot\|_{A}$ on $H A(\Omega)$. This means that (11) is bounded for any $\gamma \in A W(\Omega)$ and $g \in U(\Omega)$ and $k \in \mathbb{N}$. Moreover, the map $A W(\Omega) \ni \gamma \longmapsto K_{\gamma} \in$ $H A(\Omega)$ is analytic. These are the contents of Theorem 5.1 in [2], p. 131, where the explicit development

$$
K_{(g+h) \gamma}=K_{g \gamma}+\sum_{k=1}^{\infty}(-1)^{k} K_{g, \gamma}^{(k)} h^{(k)}
$$




$$
\begin{gathered}
\gamma \in A W(\Omega), \quad g \in U(\Omega), \quad h \in B_{g}, \\
B_{g}=B_{i(g) / 2}(0)=\left\{h \in L^{\infty}(\Omega):\|h\|_{\infty}<\frac{i(g)}{2}\right\},
\end{gathered}
$$

is also provided. The series on the right hand side of (12) converges uniformly on $B_{g}$ with respect to the semi-norm $\|\cdot\|_{A}$ on $H A(\Omega)$, for any compact subset $A \subset \Omega$. Consequently, the $k$-th derivative of the map $U(\Omega) \ni g \longmapsto K_{g \gamma} \in H A(\Omega)$ is

$$
D_{g}^{(k)} K_{g \gamma}\left(h_{1}, \cdots, h_{k}\right)=(-1)^{k} \sum_{\sigma \in \Sigma_{k}} K_{g, \gamma}^{(k)}\left(h_{\sigma(1)}, \cdots, h_{\sigma(k)}\right)
$$

where $\Sigma_{k}$ is the permutation group of order $k$ !. Cf. Corollary 5.1 in [2], p. 134.

By a result of N. Kerzman (cf. [13]), if $\Omega \subset \mathbb{C}^{n}$ is a smoothly bounded strictly pseudoconvex domain then its (ordinary) Bergman kernel $K_{1}(\zeta, z)$ is smooth up to the boundary, that is, $K_{1} \in C^{\infty}(\bar{\Omega} \times$ $\bar{\Omega} \backslash \Delta)$ where $\Delta \subset \partial \Omega \times \partial \Omega$ is the diagonal of the boundary. We conjecture that $K_{\gamma} \in C^{\infty}(\bar{\Omega} \times \bar{\Omega} \backslash \Delta)$ for any weight $\gamma \in A W(\Omega)$ for which the solution to the $\bar{\partial}$-Neumann problem with weights is available, cf. J.J. Kohn [38] and T-V. Khanh [39].

\subsection{Weighted Ramadanov Theorem}

Let $\Omega \subset \mathbb{C}^{n}$ be a bounded domain. By a classical result due to I. Ramadanov (cf. [40]), for any increasing sequence of domains

$$
\Omega_{v} \subset \Omega, \quad \Omega_{v} \subset \subset \Omega_{v+1}, \quad v \geq 1, \quad \Omega=\bigcup_{v=1}^{\infty} \Omega_{v},
$$

one has $K_{\Omega_{v}} \rightarrow K_{\Omega}$ as $v \rightarrow \infty$, uniformly on compact subsets of $\Omega \times \Omega$. Cf. also S. Krantz [32] for a qualitatively new proof of Ramadanov's theorem and further generalizations.

The extension of Ramadanov's theorem to weighted Bergman kernels was obtained by Z. Pasternak-Winiarski and P.M. Wójcicki [41]. Precisely, let $\gamma \in A W(\Omega)$ and $\gamma_{v} \in A W\left(\Omega_{v}\right)$, where each $\gamma_{\nu}$ is thought of as extended by $\gamma$ to the whole of $\Omega$. We also assume that

$$
\begin{gathered}
\text { (i) } \forall v \in \mathbb{N}, \quad \exists N=N(v) \in \mathbb{N}, \quad \forall \mu \geq N(v), \quad \forall z \in \Omega_{v} \text { : } \\
\Omega_{v} \subset \Omega_{\mu}, \quad \gamma_{v}(z) \leq \gamma_{\mu}(z) \leq \gamma(z), \\
\text { (ii) } \gamma_{v}(z) \rightarrow \gamma(z) \text { as } v \rightarrow \infty, \quad \text { for a.e. } z \in \Omega .
\end{gathered}
$$

Under these assumptions, the result in [42] is that

$$
\lim _{\nu \rightarrow \infty} K_{\gamma_{v}}=K_{\gamma}
$$

locally uniformly on $\Omega \times \Omega$. Cf. Theorem 4 in [43], pp. 4-5. A direct proof of (13), dissimilar to that in [32], is provided in [43]. It should be observed that (13) may also be proved by applying (under an appropriate monotonicity assumption on the sequence of weights) the classical (unweighted) Ramadanov theorem to the Bergman kernel $K_{\Omega_{m}}$ of the domain

$$
\Omega_{m}=\left\{(z, \xi) \in \Omega \times \mathbb{C}^{m}:|\xi|^{2 m}<\gamma(z)\right\}
$$

merely because $K_{\gamma}$ and $K_{\Omega_{m}}$ are quantitively related by

$$
K_{\gamma}(z, w)=\frac{\pi^{m}}{m !} K_{\Omega_{m}}((z, 0),(w, 0))
$$


for any $z, w \in \Omega$. The domain $\Omega_{m}$ is referred to by us as a suspension of $\Omega$, by following the terminology introduced by I. Naruki (cf. [23]). The construction (14) and the result (15) are due to F. Forelli and W. Rudin (cf. [20]) and E. Ligocka (cf. [19]). The paper [20] was published in 1974, while I. Naruki's work [23] saw the light of print in 1976. Nevertheless, C. Fefferman acknowledges (in his work [15], introducing the celebrated Fefferman metric) [23] as a source of inspiration for his construction of the potential

$$
U(\zeta, z)=|\zeta|^{2 /(n+1)} K(z, z)^{-1 /(n+1)}
$$

determining a semi-Kählerian metric

$$
G=\sum_{A, B=1}^{n+1} \frac{\partial^{2} U}{\partial z_{A} \partial \bar{z}_{B}} d z_{A} \odot d \bar{z}_{B}
$$

on $\Omega \times(\mathbb{C} \backslash\{0\})$. If $\mathbf{j}: \Omega \times S^{1} \rightarrow \Omega \times(\mathbb{C} \backslash\{0\})$ is the inclusion then $\mathbf{j}^{*} G$ is a degenerate $(0,2)$-tensor field on $\Omega \times S^{1}$, yet there is a Lorentzian metric $F$ on $\partial \Omega \times S^{1}$ (the Fefferman metric of $\Omega$ ) such that $\left(\mathbf{j}^{*} G\right)_{(z, \zeta)}$ tends to $F_{\left(z_{0}, \zeta_{0}\right)}$ as $\Omega \times S^{1} \ni(z, \zeta) \rightarrow\left(z_{0}, \zeta_{0}\right) \in \partial \Omega \times S^{1}$. The construction only works for smoothly bounded strictly pseudoconvex domains $\Omega \subset \mathbb{C}^{n}$ (cf. again [15]). Successively the Fefferman metric $F \in \operatorname{Lor}\left(\partial \Omega \times S^{1}\right)$ was intensely studied (cf. [44], p. 109-156, for its main properties as known up to the year 2006) and appeared to be a tool of first magnitude (e.g., L. Koch used (cf. [45]) the description of Chern-Moser chains on $\partial \Omega$ as projections by $\partial \Omega \times S^{1} \rightarrow \partial \Omega$ of null geodesics of $F$ to give a much simplified proof of the result by $\mathrm{H}$. Jacobowitz (cf. [46]) that two close-by points on $\partial \Omega$ may be joined by a chain). Therefore the "suspension" of a variable (leading to the potential $U(\zeta, z)$ and then to $F$ ) turned out to be yet another brilliant idea of $C$. Fefferman, an eminent scholar of twentieth century mathematics, that he claims to have taken from [23], as mentioned above. "Suspended" domains of the sort in (14) will be encountered again in Section 5 of the present survey (and used to relate the $|\varphi|^{m}$-Bergman kernel of $\Omega=\{\varphi<0\}$ to the ordinary Bergman kernel of $\Omega_{m}$ ).

We close this section by reporting briefly on a generalization of the Forelli-Rudin-Ligocka-Naruki suspension of a variable method, due to M. Englis and G. Zhang (cf. [47]) which looks very promising in terms of further (Fefferman-like) differential geometric applications. Let $\Omega \subset \mathbb{C}^{n}$ be a domain and $\gamma \in W(\Omega)$ such that $\gamma^{v} \in A W(\Omega)$ for every $v \in \mathbb{N}$. Let $F=G / H$ be an irreducible bounded symmetric domain and let us set

$$
\Omega^{F}=\left\{(z, \xi) \in \Omega \times \mathbb{C}^{d}: \frac{1}{\sqrt{\gamma(z)}} \xi \in F\right\} .
$$

The space $\mathcal{P}$ of holomorphic polynomials on $F$ decomposes multiplicity-free with respect to the $H$-action as

$$
\mathcal{P}=\bigoplus_{\mathbf{m}=\text { partition }} \mathcal{P}_{\mathbf{m}}
$$

Let $K_{\mathbf{m}}$ be the reproducing kernel of the subspace $\mathcal{P}_{\mathbf{m}}$ with respect to the Fischer inner product. Then the Bergman kernel of the domain $\Omega^{F}$ is given by the series

$$
K^{\Omega^{F}}(z, \xi, \zeta, \eta)=\frac{1}{\operatorname{vol}(F)} \sum_{\mathbf{m}}(p)_{\mathbf{m}} K_{\mathbf{m}}(\xi, \eta) K_{\gamma^{d+|\mathbf{m}|}}(z, \zeta) .
$$

The formula (16) may be used to recover the results by F. Forelli and W. Rudin [20], E. Ligocka [19], and G. Roos [48] (the last quoted paper extends at its turn a method by H.P. Boas and S. Fu and E.J. Straube [49], which will be met again in this survey in connection with the work by Z. Huo [50]). 


\section{Reproducing Kernels and Quantization of States}

\subsection{Hilbert Spaces of $L^{2}$ Holomorphic Sections}

Let $M$ be a complex $n$-dimensional manifold. Let $E$ be a holomorphic line bundle over $M$, with projection $\pi: E \rightarrow M$. Let us fix a local trivialization atlas $\left\{\left(U_{\alpha}, \Phi_{\alpha}\right)\right\}_{\alpha \in J}$ of $E$

$$
\Phi_{\alpha}: \pi^{-1}\left(U_{\alpha}\right) \rightarrow U_{\alpha} \times \mathbb{C}, \quad \alpha \in J
$$

such that each $U_{\alpha}$ is the domain of a local complex coordinate system $\left(U_{\alpha}, z_{\alpha}^{1}, \cdots, z_{\alpha}^{n}\right)$ on $M$. Let $\bar{\partial}_{E}$ : $C^{\infty}(E) \rightarrow C^{\infty}\left(\Lambda^{0,1}(M) \otimes E\right)$ be the first-order differential operator associated to the holomorphic structure of $E$. If $z \in M$ and $\alpha \in J$ is an index such that $z \in U_{\alpha}$, then

$$
\begin{gathered}
\left(\bar{\partial}_{E} S\right)_{z}=\left(\bar{\partial} f_{\alpha} \otimes s_{\alpha}\right)_{z}, \\
\left.s\right|_{U_{\alpha}}=f_{\alpha} s_{\alpha}, \quad f_{\alpha} \in C^{\infty}\left(U_{\alpha}, \mathbb{C}\right), \quad s_{\alpha}(\zeta)=\Phi_{\alpha}^{-1}(\zeta, 1), \quad \zeta \in U_{\alpha} .
\end{gathered}
$$

In particular $s_{\alpha}$ is a local holomorphic section in $E$-that is, $\bar{\partial}_{E} s_{\alpha}=0$. Next, $\Lambda^{n, 0}(M) \otimes E \rightarrow M$ is a holomorphic line bundle. Let $H$ be a Hermitian bundle metric on $E$, and given $\omega \in \mathcal{O}\left(\Lambda^{n, 0}(M) \otimes E\right)$ let $H^{*}(\omega, \omega)$ be the (globally defined) complex-valued differential form of type $(n, n)$

$$
\begin{gathered}
\left.H^{*}(\omega, \omega)\right|_{U_{\alpha}}=\left|\Psi_{\alpha}\right|^{2} \gamma_{\alpha} d z_{\alpha}^{1} \wedge \cdots \wedge d z_{\alpha}^{n} \wedge d \bar{z}_{\alpha}^{1} \wedge \cdots \wedge d \bar{z}_{\alpha}^{n} \\
\left.\omega\right|_{U_{\alpha}}=\Psi_{\alpha} s_{\alpha} \otimes d z_{\alpha}^{1} \wedge \cdots \wedge d z_{\alpha}^{n} \\
\Psi_{\alpha} \in \mathcal{O}\left(U_{\alpha}\right), \quad \gamma_{\alpha}=H\left(s_{\alpha}, s_{\alpha}\right) .
\end{gathered}
$$

Let $\mathcal{M}$ be the space

$$
\mathcal{M}=\left\{\omega \in \mathcal{O}\left(\Lambda^{n, 0}(M) \otimes E\right): \int_{M} H^{*}(\omega, \omega)<\infty\right\} .
$$

From a physical viewpoint, one thinks of $M$ as the classical phase space (the phase space of a classical physical system). The complex projective space $\mathbb{C P}(\mathcal{M})$ is then the quantum phase space and the quantization of classical states amounts to building an embedding $\mathcal{K}: M \rightarrow \mathbb{C P}(\mathcal{M})$. By a result of K. Gawędzki (cf. [26]), $\mathcal{M}$ is a complex Hilbert space with the inner product

$$
\begin{gathered}
\langle\omega, \varphi\rangle=i^{n^{2}} \int_{M} H^{*}(\omega, \varphi), \\
\left.H^{*}(\omega, \varphi)\right|_{U_{\alpha}}=\Psi_{\alpha} \bar{u}_{\alpha} \gamma_{\alpha} d z_{\alpha}^{1} \wedge \cdots \wedge d z_{\alpha}^{n} \wedge d \bar{z}_{\alpha}^{1} \wedge \cdots \wedge d \bar{z}_{\alpha}^{n}, \\
\left.\varphi\right|_{U_{\alpha}}=u_{\alpha} s_{\alpha} \otimes d z_{\alpha}^{1} \wedge \cdots \wedge d z_{\alpha}^{n}, \quad u_{\alpha} \in \mathcal{O}\left(U_{\alpha}\right) .
\end{gathered}
$$

To show how reproducing kernels occur in ordinary quantum mechanics we follow the work by A. Odzijewicz (cf. [3]), where one works under the following additional requirement:

$$
\forall z, \zeta \in M, \quad \exists \omega, \varphi \in \mathcal{M}: \operatorname{det}\left[\begin{array}{ll}
\Psi_{\alpha}(z) & \Psi_{\beta}(\zeta) \\
u_{\alpha}(z) & u_{\beta}(\zeta)
\end{array}\right] \neq 0
$$

whenever $\alpha, \beta \in J$ are chosen such that $z \in U_{\alpha}$ and $\zeta \in U_{\beta}$. According to [3], pp. 579-580, requirement (17) guarantees that $\mathcal{M}$ is "sufficiently ample". Certainly (17) is a global requirement (it does not depend upon the local representation of the $E$-valued $(n, 0)$-forms $\omega$ and $\varphi$ about the points $z$ and $\zeta)$. 
Let $z \in M$ and let $\alpha \in J$ such that $z \in U_{\alpha}$. We may then consider the evaluation functional

$$
\delta_{z}^{\alpha}: \mathcal{M} \rightarrow \mathbb{C}, \quad \delta_{z}^{\alpha}(\omega)=\Psi_{\alpha}(z),
$$

for any $\omega \in \mathcal{M}$ locally represented as $\left.\omega\right|_{U_{\alpha}}=\Psi_{\alpha} s_{\alpha} d \zeta_{\alpha}^{1} \wedge \cdots \wedge d \zeta_{\alpha}^{n}$. One has:

Lemma 1 (K. Gawę̧dzki, [26]). $\left|\Psi_{\alpha}(z)\right| \leq C_{\alpha}\|\omega\|$.

Lemma 1 yields the continuity of the map (18), so Riesz theorem applies. Hence, there is $k_{z, \bar{\alpha}} \in \mathcal{M}$ such that

$$
\delta_{z}^{\alpha}(\omega)=\left\langle\omega, k_{z, \bar{\alpha}}\right\rangle=i^{n^{2}} \int_{M} H^{*}\left(\omega, k_{z, \bar{\alpha}}\right)
$$

and

$$
\begin{gathered}
\left.k_{z, \bar{\alpha}}\right|_{U_{\beta}}=\overline{K_{\alpha \bar{\beta}}(z, \cdot)} s_{\beta} \otimes d \zeta_{\beta}^{1} \wedge \cdots \wedge d \zeta_{\beta}^{n}, \\
\left.H^{*}\left(\omega, k_{z, \bar{\alpha}}\right)\right|_{U_{\beta}}=K_{\alpha \bar{\beta}}(z, \cdot) \Psi_{\beta} \gamma_{\beta} d \zeta_{\beta}^{1} \wedge \cdots \wedge d \zeta_{\beta}^{n} \wedge d \bar{\zeta}_{\beta}^{1} \wedge \cdots \wedge d \bar{\zeta}_{\beta}^{n} .
\end{gathered}
$$

As we shall see in a moment, the functions $K_{\alpha \bar{\beta}}(z, \zeta)$ defined by (20) are reproducing kernels and their properties will be investigated shortly. Note first that:

(i) For every $z \in M$ and every $\alpha \in J$ such that $z \in U_{\alpha}$, the holomorphic section $k_{z, \bar{\alpha}}$ is not the zero section. Indeed, if $k_{z, \bar{\alpha}}=0$ then (by (19)) $\omega(z)=0$ for every $\omega \in \mathcal{M}$. Moreover, according to Odzijewicz's ampleness assumption (17), for every $\zeta \in M$ we may consider $\omega, \varphi \in \mathcal{M}$ such that

$$
\operatorname{det}\left[\begin{array}{ll}
\Psi_{\alpha}(z) & \Psi_{\beta}(\zeta) \\
u_{\alpha}(z) & u_{\beta}(\zeta)
\end{array}\right] \neq 0
$$

provided that $\zeta \in U_{\beta}$, in contradiction with $\Psi_{\alpha}(z)=0$ and $u_{\alpha}(z)=0$.

As another important property of $k_{z, \bar{\alpha}}$, leading to quantization of classical states, one has (ii)

$$
k_{z, \bar{\beta}}=\overline{g_{\alpha \beta}(z)} \frac{\overline{\partial \zeta_{\alpha}}(z)}{\partial \zeta_{\beta}} k_{z, \bar{\alpha}}
$$

for every $z \in U_{\alpha} \cap U_{\beta}$, where

$$
\frac{\partial \zeta_{\alpha}}{\partial \zeta_{\beta}}(z)=\operatorname{det}\left[\frac{\partial \zeta_{\alpha}^{j}}{\partial \zeta_{\beta}^{k}}(z)\right] .
$$

Indeed, (21) follows from (19) and

$$
\Psi_{\beta}=g_{\alpha \beta} \frac{\partial \zeta_{\alpha}}{\partial \zeta_{\beta}} \Psi_{\alpha}
$$

At this point one may consider the map

$$
\mathcal{K}: M \rightarrow \mathbb{C P}(\mathcal{M}), \quad \mathcal{K}(z)=\left[k_{z, \bar{\alpha}}\right], \quad z \in M,
$$

provided that $z \in U_{\alpha}$. Here $[\omega]=\{\lambda \omega: \lambda \in \mathbb{C} \backslash\{0\}\} \in \mathbb{C P}(\mathcal{M})$ is the (projective) ray represented by $\omega \in \mathcal{M} \backslash\{0\}$. The physical meaning of $\mathbb{C P}(\mathcal{M})$ is that in quantum theory the wave functions $\omega \in$ $\mathcal{M}$ and $\lambda \omega \in \mathcal{M}$ (or $\Psi_{\alpha}$ and $\lambda \Psi_{\alpha}$ with respect to the local description $\left.\omega\right|_{U_{\alpha}}=\Psi_{\alpha} s_{\alpha} \otimes d z_{\alpha}^{1} \wedge \cdots \wedge d z_{\alpha}^{n}$ ) represent the same physical state for any $\lambda \in \mathbb{C} \backslash\{0\}$. A normalized wave function, that is, $\omega \in \mathcal{M}$ with $\langle\omega, \omega\rangle=1$, may be chosen in a ray, yet the normalization procedure determines $\omega$ only up to a factor $\lambda=e^{i \phi} \in \mathrm{U}(1)$. Here $\phi$ is referred to as the global phase. The phase of a ray is not observable.

As a consequence of (21), the definition of $\mathcal{K}(z)$ does not depend upon the choice of $\alpha \in J$ with $z \in U_{\alpha}$ 
Formula (19) for $\omega=k_{z, \bar{\alpha}}$ yields $\left\|k_{z, \bar{\alpha}}\right\|^{2}=\overline{K_{\alpha \bar{\alpha}}(z, z)}$ hence (iii)

$$
K_{\alpha \bar{\alpha}}(z, z)>0
$$

Property (22) is referred to as positivity (of the kernel $K_{\alpha \bar{\alpha}}(z, \zeta)$ ). Again, by (19) for $\omega=k_{\zeta, \bar{\beta}}$ yields

$$
\begin{gathered}
\left\langle k_{\zeta, \bar{\beta}}, k_{z, \bar{\alpha}}\right\rangle=\overline{K_{\beta \bar{\alpha}}(\zeta, z)}, \\
\left.k_{\zeta, \bar{\beta}}\right|_{U_{\alpha}}=\overline{K_{\beta \bar{\alpha}}(\zeta \cdot)} s_{\alpha} \otimes d \zeta_{\alpha}^{1} \wedge \cdots \wedge d \zeta_{\alpha}^{n},
\end{gathered}
$$

or (iv)

$$
K_{\beta \bar{\alpha}}(\zeta, z)=\left\langle k_{z, \bar{\alpha}}, k_{\zeta, \bar{\beta}}\right\rangle
$$

for all $z \in U_{\alpha}$ and $\zeta \in U_{\beta}$. One refers to (23) as the reproducing property. Next let us start by restricting

$$
k_{z, \bar{\beta}}=\overline{g_{\alpha \beta}(z)} \overline{\frac{\partial \zeta_{\alpha}}{\partial \zeta_{\beta}}(z)} k_{z, \bar{\alpha}}, \quad z \in U_{\alpha} \cap U_{\beta},
$$

to $U_{\gamma} \cap U_{\delta}$. One obtains

$$
\begin{gathered}
\overline{K_{\beta \bar{\gamma}}(z, \cdot)} s_{\gamma} \otimes d \zeta_{\gamma}^{1} \wedge \cdots \wedge d \zeta_{\gamma}^{n}= \\
=\overline{g_{\alpha \beta}(z)} \overline{\frac{\partial \zeta_{\alpha}}{\partial \zeta_{\beta}}(z)} \overline{K_{\alpha \bar{\delta}}(z, \cdot)} s_{\delta} \otimes d \zeta_{\delta}^{1} \wedge \cdots \wedge d \zeta_{\delta}^{n}
\end{gathered}
$$

or $(\mathrm{v})$

$$
K_{\beta \bar{\gamma}}(z, \zeta)=g_{\alpha \beta}(z) \frac{\partial \zeta_{\alpha}}{\partial \zeta_{\beta}}(z) \overline{g_{\delta \gamma}(\zeta)} \overline{\frac{\partial \zeta_{\delta}}{\partial \zeta_{\gamma}}(\zeta)} K_{\alpha \bar{\delta}}(z, \zeta)
$$

for any $\zeta \in U_{\gamma} \cap U_{\delta}$. Formula (24) is referred to as the transformation law (of the kernel $K_{\alpha \bar{\beta}}(z, \zeta)$ ). Moreover, (vi) there is a globally defined $(0,2)$ tensor field $g$ on $M$ such that

$$
\left.g\right|_{U_{\alpha}}=\sum_{j, k=1}^{n} \frac{\partial^{2} \log K_{\alpha \bar{\alpha}}(z, z)}{\partial z_{\alpha}^{j} \partial \bar{z}_{\alpha}^{k}} d z_{\alpha}^{j} \odot d \bar{z}_{\alpha}^{k}
$$

for every $\alpha \in J$. By a result of A. Odzijewicz (cf. Proposition 2 in [9], p. 582) the following statements are equivalent:

(a) $\mathcal{K}: M \rightarrow \mathbb{C P}(\mathcal{M})$ is one-to-one.

(b) For all $z_{1}, z_{2} \in M$ there are $\zeta_{1}, \zeta_{2} \in M$ such that

$$
\operatorname{det}\left[\begin{array}{cc}
K_{\gamma \bar{\alpha}}\left(\zeta_{1}, z_{1}\right) & K_{\gamma \bar{\beta}}\left(\zeta_{1}, z_{2}\right) \\
K_{\delta \bar{\alpha}}\left(\zeta_{2}, z_{1}\right) & K_{\delta \bar{\beta}}\left(\zeta_{2}, z_{2}\right)
\end{array}\right] \neq 0
$$

provided that $\zeta_{1} \in U_{\gamma}, \zeta_{2} \in U_{\delta}, z_{1} \in U_{\alpha}$ and $z_{2} \in U_{\beta}$.

(c) The ampleness condition (18) is fulfilled.

The fundamental result obtained in [9] is as follows.

Theorem 2. The following statements are equivalent:

(1) The map $\mathcal{K}: M \rightarrow \mathbb{C P}(\mathcal{M})$ is a holomorphic embedding.

(2) The condition (26) is fulfilled and the $(0,2)$-tensor field $g$ given by (25) is positive definite. 
Cf. Proposition 3 in [9], p. 583. By Theorem 2 if $\mathcal{K}: M \rightarrow \mathbb{C P}(\mathcal{M})$ is a holomorphic embedding then $g$ is a Riemannian metric on $M$, referred to as the Bergman metric. Of course, metrics derived from a potential, such as the metric $g$ locally given by (25), are Kählerian. The proof of Theorem 2 is an easy adaptation of the proof in [51]. At the time when S. Kobayashi's paper [51] was written, locally conformal Kähler (l.c.K.) metrics were unknown to the community devoted to the study of complex analysis and geometry. To set matters into a correct historical perspective, we should mention that 1.c.K. metrics were introduced by P. Libermann (cf. [52]) in 1954-1955 (while [51] was published in 1959). Additionally, the first example of a Hermitian metric (on a compact complex manifold) which is 1.c.K. but not Kähler was discovered in 1954 by W.M. Boothby (cf. [53]) yet the example was not recognized as such until 1976, with the publication of I. Vaisman's work [54]. The main obstacle for the development of a theory of 1.c.K. manifolds was perhaps T. Aubin's "result" (cf. [55]) that any compact 1.c.K. manifold should be Kählerian. T. Aubin's statement was eventually proven wrong (cf. [54]) by exhibiting Boothby's example (cf. [53]): the complex Hopf manifold $H^{n}(\lambda)=\left(\mathbb{C}^{n} \backslash\{0\}\right) / \Delta_{\lambda} \approx S^{2 n-1} \times S^{1}, 0<|\lambda|<1$ (cf. e.g., [56], Vol. II, p. 137) carries the l.c.K. metric $|z|^{-2} \sum_{j=1}^{n} d z^{j} \odot d \bar{z}^{j}$ yet admits no globally defined Kähler metric for topological reasons (its first Betti number is $b_{1}\left[H^{n}(\lambda)\right]=1$, while the odd-order Betti numbers $b_{2 p+1}(M)$ of a compact complex manifold $M$ should be even - cf. e.g., [57], p. 178). Therefore, the quantization procedure devised by A. Odzijewicz (cf. [9]) will not work when $M$ is a complex manifold admitting no Kählerian metric. Are there any mechanical systems whose classical phase space is a complex manifold of that sort (i.e., not satisfying the topological constraints of a Kählerian manifold)? The problem of the quantization of classical states when the phase space is a non-Kähler l.c.K. manifold is open.

Example 5 (Classical phase space is a domain $\Omega \subset \mathbb{C}^{n}$ ). Let $M=\Omega$ be a domain in $\mathbb{C}^{n}$ and let $E \rightarrow \Omega$ be the trivial complex line bundle, that is, $E=\Omega \times \mathbb{C}$, endowed with the Hermitian bundle metric $H$. Let $s \in \mathcal{O}(E)$ be given by $s(z)=(z, 1)$ for any $z \in \Omega$, and let us set $\gamma=H(s, s)$ so that $\gamma \in W(\Omega)$. Then, each $\omega \in \mathcal{M}$ may be represented as $\omega=\Psi s \otimes d \zeta^{1} \wedge \cdots d \zeta^{n}$ for some $\Psi \in \mathcal{O}(\Omega)$ and

$$
\begin{gathered}
d \zeta^{j} \wedge d \bar{\zeta}^{j}=-2 i d x^{j} \wedge d y^{j}, \quad \zeta^{j}=x^{j}+i y^{j}, \\
\langle\omega, \omega\rangle=i^{n^{2}} \int_{\Omega} H^{*}(\omega, \omega)= \\
=i^{n^{2}} \int_{\Omega}|\Psi|^{2} \gamma d \zeta^{1} \wedge \cdots \wedge d \zeta^{n} \wedge d \bar{\zeta}^{1} \wedge \cdots \wedge d \bar{\zeta}^{n}=2^{n}\|\Psi\|_{\gamma}^{2}
\end{gathered}
$$

so that $\Psi \in L^{2} H(\Omega, \gamma)$ and the map

$$
\mathcal{M} \rightarrow L^{2} H(\Omega, \gamma), \quad \omega \mapsto 2^{-n / 2} \Psi,
$$

is an isometry. Next (by K. Gawędzki's Lemma 1) the weight $\gamma=H(s, s)$ is admissible, that is, $\gamma \in A W(\Omega)$ and

$$
K_{\alpha \bar{\alpha}}(z, \zeta)=2^{-n} K_{\gamma}(z, \zeta)
$$

for any $z, \zeta \in \Omega$. Here, to establish a connection with the previously adopted notation, we let $J=\{\alpha\}$ be the index set consisting of but one symbol $\alpha$ and $\Phi_{\alpha}=1_{E}$.

Example 6 (Segal-Bargmann space). Let $\alpha>0$ and let $\gamma_{\alpha} \in W\left(\mathbb{C}^{n}\right)$ be given by $\gamma_{\alpha}(z)=\exp (-$ $\left.\alpha|z|^{2}\right)$ for any $z \in \mathbb{C}^{n}$. Then, $L^{2} H\left(\mathbb{C}^{n}, \gamma_{\alpha}\right)$ is the Segal-Bargmann-Fock space of quantum mechanics (with parameter $\alpha$ ), cf. G.B. Folland [58]. This is an RKH space so $\gamma_{\alpha} \in A W\left(\mathbb{C}^{n}\right)$ and the corresponding $\gamma_{\alpha}$-Bergman kernel is shown to be

$$
K_{\gamma_{\alpha}}(z, \zeta)=\left(\frac{\alpha}{\pi}\right)^{n} \exp (z \cdot \bar{\zeta}), \quad z, \zeta \in \mathbb{C}^{n}
$$


Cf. also V. Bargmann [59]. A unit vector in $L^{2} H\left(\mathbb{C}^{n}, \gamma_{\alpha}\right)$ is thought of as the wave function of a quantum particle moving in configuration space $\mathbb{R}^{n}$ (while $\mathbb{C}^{n}$ is the classical phase space).

If $1 \leq p<\infty$, let $L^{p}\left(\mathbb{C}^{n}, \gamma_{\alpha}\right)$ consist of all measurable functions such that $|f|^{p} \in L^{1}\left(\mathbb{C}^{n}, \gamma_{\alpha} d \mu\right)$, and let us set

$$
L^{p} H\left(\mathbb{C}^{n}, \gamma_{\alpha}\right)=\mathcal{O}\left(\mathbb{C}^{n}\right) \cap L^{p}\left(\mathbb{C}^{n}, \gamma_{\alpha}\right)
$$

$L^{p}\left(\mathbb{C}^{n}, \gamma_{\alpha}\right)$ and $L^{p} H\left(\mathbb{C}^{n}, \gamma_{\alpha}\right)$ are Banach spaces when equipped with norm $\|f\|_{p, \gamma_{\alpha}}=$ $\left[\int_{\mathbb{C}^{n}}|f(\zeta)|^{p} \gamma_{\alpha}(\zeta) d \mu(\zeta)\right]^{1 / p}$. The orthogonal projection

$$
P_{\gamma_{\alpha}} f(z)=\int_{\mathbb{C}^{n}} f(\zeta) K_{\gamma_{\alpha}}(z, \zeta) \gamma_{\alpha}(\zeta) d \mu(\zeta)
$$

is (by construction) a bounded operator from $L^{2}\left(\mathbb{C}^{n}, \gamma_{\alpha}\right)$, yet may be unbounded as an operator from $L^{p}\left(\mathbb{C}^{n}, \gamma_{\alpha}\right)$ with $p \neq 2$.

By a result of S. Janson and J. Peetre and R. Rochberg (cf. [60]) if $\alpha \in \mathbb{R}, \beta>0$, and $1 \leq p<\infty$ satisfy $\beta p>\alpha$, then $P_{\gamma_{\beta}}$ is bounded as an operator from $L^{p}\left(\mathbb{C}^{n}, \gamma_{\alpha}\right)$ into $L^{p}\left(\mathbb{C}^{n}, \gamma_{\sigma}\right)$ where $1 / \sigma=4(\beta p-$ $\alpha) /\left(p^{2} \beta^{2}\right)$.

H. Bommier-Hato, M. Englis, and El-H. Youssfi considered (cf. [61]) the family of weights $\gamma_{\alpha, m} \in W\left(\mathbb{C}^{n}\right)$, $\alpha>0, m>0$, given by $\gamma_{\alpha, m}(z)=\exp \left(-\alpha|z|^{2 m}\right)$ and described the relationship among $\alpha>0, \beta>0, \sigma>0$, and $1 \leq p, q<\infty$ such that the projection $P_{\gamma_{\beta, m}}$ be a bounded operator from $L^{2}\left(\mathbb{C}^{n}, \gamma_{\alpha, m}\right)$ into $L^{q}\left(\mathbb{C}^{n}, \gamma_{\sigma, m}\right)$ (thus generalizing the result by $S$. Janson et al., cf. op. cit.).

Going back to the (now) classical situation contemplated by I.E. Segal (cf. [62-64]) and V. Bargmann (cf. $[59,65,66])$ we recall the Segal-Bargmann transform (the coherent state transform) A from $L^{2}\left(\mathbb{R}^{n}\right)$ into $\mathcal{O}\left(\mathbb{C}^{n}\right)$

$$
\begin{gathered}
(A f)(z)=\int_{\mathbb{R}^{n}} A(z, x) f(x) d x, \\
A(z, x)=\frac{\rho_{1}(z-x)}{\sqrt{\rho_{1}(x)}}, \quad f \in L^{2}\left(\mathbb{R}^{n}\right), \quad z \in \mathbb{C}^{n},
\end{gathered}
$$

where

$$
\rho_{t}(x)=(2 \pi t)^{-n / 2} \exp \left(-\frac{|x|^{2}}{2 t}\right)
$$

is the standard heat kernel on $\mathbb{R}^{n}$ and $\rho_{t}(z)$ denotes the analytic continuation of $\rho_{t}$ to $\mathbb{C}^{n}$. The main result by I.E. Segal and V. Bargmann is (cf. op. cit.) that $A$ is an isometric isomorphism of $L^{2}\left(\mathbb{R}^{n}\right)$ onto the Hilbert space $L^{2} H\left(\mathbb{C}^{n}, \pi^{-n} \gamma_{1}\right)$. A generalization of this situation was taken up by B.C. Hall (cf. [67]), who replaced $\mathbb{R}^{n}$ by a compact, connected Lie group $K$ and $\mathbb{C}^{n}$ by the complexification $G$ of $K$. By a result in [67] the heat kernel $\rho_{t}$ has a unique analytic continuation from $K$ to $G$, and one may set

$$
\begin{gathered}
A_{t}(g, x)=\frac{\rho_{t}\left(x^{-1} g\right)}{\rho_{t}(x)}, x \in K, g \in G, \\
\left(A_{t} f\right)(g)=\int_{K} A_{t}(g, x) f(x) d x, \quad f \in L^{2}(K), g \in G .
\end{gathered}
$$

As $\rho_{t}\left(x^{-1} g\right)$ is (by construction) a holomorphic function of $g, A_{t}$ maps $L^{2}(K)$ into $\mathcal{O}(G)$ and actually (by Theorem 1 in [67], p. 105) for each $t>0$ the coherent state transform $A_{t}$ is an isometric isomorphism of $L^{2}(K)$ onto $L^{2} H\left(G, \mu_{t}\right)$, where $\mu_{t}$ (the analog to the Gaussian measure on $\mathbb{C}^{n}$ ) is the fundamental solution at the identity of the following equation on $G$

$$
\frac{d u}{d t}=\frac{1}{4} \Delta_{G} u
$$

Additionally, if $\left\{X_{i}, J X_{i}: 1 \leq i \leq n\right\}$ is a basis of the Lie algebra $\mathfrak{g}$ of $G$, then $\Delta_{G} \equiv \sum_{i=1}^{n}\left(X_{i}^{2}+\left(J X_{i}\right)^{2}\right)$ (the Laplace-Beltrami operator associated to the left invariant Riemannian metric on G determined by $\left\{X_{i}, J X_{i}\right\}$ ). 
Cf. E. Nelson [68] for the construction and basic properties of $\mu_{t}$. By Theorem 5 in [67], p. 127, the $\mu_{t}$-Bergman kernel $K_{\mu_{t}}(g, h)$ is given by the beautiful formula

$$
K_{\mu_{t}}(g, h)=\int_{K} \frac{\rho_{t}\left(x^{-1} g\right) \overline{\rho_{t}\left(x^{-1} h\right)}}{\rho_{t}(x)} d x, \quad g, h \in G .
$$

Cf. also S. van Leeuwen [69], S. Barbier [70], and A. Yamamori [71].

\subsection{Hermitian Geometry of Complex Line Bundles}

Given $v, w \in E_{z}$ with $z \in M$ and $v \neq 0$ let $\lambda=w / v \in \mathbb{C}$ be the complex number uniquely determined by $w=\lambda v$. Additionally, if $s: M \rightarrow E$ and $r: M \rightarrow E$ are sections with $s(z) \neq 0$ for any $z \in M$, then $r / s: M \rightarrow \mathbb{C}$ is the function given by $(r / s)(z)=r(z) / s(z)$. Let $\nabla: C^{\infty}(E) \rightarrow$ $\Omega^{1}(E)=C^{\infty}\left(T^{*}(M) \otimes E\right)$ be a connection in $E$ as a vector bundle. To every nowhere zero local section $s \in C^{\infty}(U, E)$ one associates a map

$$
\mathfrak{X}(M) \rightarrow C^{\infty}(U, \mathbb{C}), \quad X \longmapsto \frac{1}{2 \pi i} \frac{\nabla_{X} s}{s},
$$

that is a vector bundle valued differential 1-form $\alpha=\alpha(s) \in \Omega^{1}(U)$ such that

$$
\nabla_{X} s=2 \pi i \alpha(X) s
$$

for any $X \in \mathfrak{X}(M)$ and any $s \in C^{\infty}(U, E)$. If $s, r \in C^{\infty}(U, E)$ are two nowhere vanishing sections then

$$
\alpha(r)=\alpha(s)+\frac{1}{2 \pi i} \frac{1}{f} d f, \quad f=\frac{r}{s} \in C^{\infty}(U, \mathbb{C}) .
$$

Formula (27) is useful in relating the connection 1-forms $\alpha_{\beta}=\alpha\left(s_{\beta}\right) \in \Omega^{1}\left(U_{\beta}\right), \beta \in J$, by means of the transition functions $g_{\beta \gamma}: U_{\beta} \cap U_{\gamma} \rightarrow \mathbb{C} \backslash\{0\}$, that is,

$$
\alpha_{\gamma}=\alpha_{\beta}+\frac{1}{2 \pi i} \frac{1}{g_{\beta \gamma}} d g_{\beta \gamma} .
$$

While the holomorphic line bundle $E$ may be reconstructed from the transition functions $g_{\beta \gamma} \in$ $\mathcal{O}\left(U_{\beta} \cap U_{\gamma}\right)$, the connection $\nabla$ may be recovered from the connection 1-forms $\alpha_{\beta}$-that is, given a family of local 1-forms $\alpha_{\beta} \in \Omega^{1}\left(U_{\beta}\right), \beta \in J$, obeying (28) there is a unique connection $\nabla$ in $E$ such that $\alpha\left(s_{\beta}\right)=\alpha_{\beta}$ for any $\beta \in J$. Profiting from the particular situation at hand (that of complex line bundles) a beautiful explicit construction of $\nabla_{X} s$ is available; that is,

$$
\left.\left(\nabla_{X} s\right)\right|_{U_{\alpha}}=\left\{X\left(\frac{s}{s_{\beta}}\right)+2 \pi i \alpha_{\beta}(X) \frac{s}{s_{\beta}}\right\} s_{\beta} .
$$

Let us set $E^{0}=\bigcup_{z \in M}\left(E_{z} \backslash\left\{0_{z}\right\}\right)$ so that $E^{0}$ is an open subset of $E$ and the total space of a principal bundle $\mathrm{GL}(1, \mathbb{C}) \rightarrow E^{0} \rightarrow M$ with structure group $\mathrm{GL}(1, \mathbb{C})=\mathbb{C} \backslash\{0\}$ (the multiplicative nonzero complex numbers). Clearly $E$ and the associated bundle of standard fibre $\mathbb{C}$

$$
E^{0} \times_{\mathrm{GL}(1, \mathbb{C})} \mathbb{C}=\left(E^{0} \times \mathbb{C}\right) / \mathrm{GL}(1, \mathbb{C})
$$

are isomorphic vector bundles. Let us consider the holomorphic $(1,0)$-form

$$
\frac{1}{2 \pi i} \frac{1}{z} d z \in \mathcal{O}(\mathbb{C} \backslash\{0\})
$$


Then, (29) is a left invariant differential 1-form on the Lie group GL(1, $\mathbb{C})$. That is,

$$
\begin{gathered}
L_{a}^{*}\left(\frac{1}{z} d z\right)=\frac{1}{z} d z, \quad a \in \mathrm{GL}(1, \mathbb{C}), \\
L_{a}: \mathrm{GL}(1, \mathbb{C}) \rightarrow \mathrm{GL}(1, \mathbb{C}), \quad L_{a}(\lambda)=a \lambda, \quad \lambda \in \mathrm{GL}(1, \mathbb{C}) .
\end{gathered}
$$

For each $v \in E_{z}^{0}$ let

$$
\tau_{v}: \mathrm{GL}(1, \mathbb{C}) \rightarrow E^{0}, \quad \tau_{v}(\lambda)=\lambda v, \quad \lambda \in \mathrm{GL}(1, \mathbb{C}),
$$

be the natural injection, so that $\tau_{v}: \mathrm{GL}(1, \mathbb{C}) \rightarrow E_{z}^{0}$ is a $C^{\infty}$ diffeomorphism. Let then $\beta_{z} \in \Omega^{1}\left(E_{z}^{0}\right)$ be the 1-form determined by

$$
\tau_{v}^{*}\left(\beta_{z}\right)=\frac{1}{2 \pi i} \frac{1}{z} d z
$$

The definition of $\beta_{z}$ does not depend upon the choice of $v \in E_{z}^{0}$. To check the statement we give $\beta_{z}$ the temporary name $\beta_{z}^{v}$. If $w \in E_{z}^{0}$ is another vector then $w=\mu v$ for some $\mu \in \mathbb{C} \backslash\{0\}$ and $\beta_{z}^{w} \in \Omega^{1}\left(E_{z}^{0}\right)$ is determined by

$$
\tau_{w}^{*}\left(\beta_{z}^{w}\right)=\frac{1}{2 \pi i} \frac{1}{z} d z
$$

Note that $\tau_{w}=\tau_{v} \circ L_{\mu}$ and then for every $\zeta \in \mathbb{C} \backslash\{0\}$

$$
\left(\tau_{w}^{*} \beta_{z}^{w}\right)_{\zeta}=\left(\beta_{z}^{w}\right)_{\zeta w} \circ\left(d_{\zeta} \tau_{w}\right)=\left(\beta_{z}^{w}\right)_{\zeta w} \circ\left(d_{\mu \zeta} \tau_{v}\right) \circ\left(d_{\zeta} L_{\mu}\right)
$$

hence

$$
\begin{aligned}
& \left(\beta_{z}^{w}\right)_{\zeta w} \circ\left(d_{\mu \zeta} \tau_{v}\right)=\left(\frac{1}{2 \pi i} \frac{1}{z} d z\right)_{\zeta} \circ\left(d_{\mu \zeta} L_{1 / \mu}\right)= \\
& =\left(\frac{1}{2 \pi i} \frac{1}{z} d z\right)_{\mu \zeta}=\left(\tau_{v}^{*} \beta_{z}^{v}\right)_{\mu \zeta}=\left(\beta_{z}^{v}\right)_{\mu \zeta v} \circ\left(d_{\mu \zeta} \tau_{v}\right)
\end{aligned}
$$

and $\tau_{w}(\zeta)=\zeta w=(\zeta \mu) v=\tau_{v}(\mu \zeta)$ hence $\beta_{z}^{w}=\beta_{z}^{v}$. Q.e.d.

Let $\alpha \in C^{\infty}\left(T^{*}\left(E^{0}\right) \otimes \mathfrak{g l}(1, \mathbb{C})\right)=\Omega^{1}\left(E^{0}\right)$ be a connection 1-form in the principal bundle $\mathrm{GL}(1, \mathbb{C}) \rightarrow E^{0} \rightarrow M$ (where $\mathfrak{g l}(1, \mathbb{C}) \approx \mathbb{C}$ is the Lie algebra of $\left.\mathrm{GL}(1, \mathbb{C})=\mathbb{C} \backslash\{0\}\right)$. That is, (i) $R_{a}^{*} \alpha=\operatorname{ad}\left(g^{-1}\right) \alpha$ for any $a \in \mathrm{GL}(1, \mathbb{R})$ and (ii) $\alpha\left(A^{*}\right)=A$ for any left invariant vector field $A \in \mathfrak{g l}(1, \mathbb{C})$. Cf. e.g., [56], Vol. I. It may be easily shown that axiom (ii) is equivalent to

$$
j_{z}^{*} \alpha=\beta_{z}, \quad z \in M,
$$

where $j_{z}: E_{x}^{0} \hookrightarrow E^{0}$ is the canonical inclusion.

Connection 1-forms may be associated to connections $\nabla$ in $E$ as follows. Let $E \rightarrow M$ be a complex line bundle. For every connection $\nabla$ in $E$ there is a unique connection 1-form $\alpha \in \Omega^{1}\left(E^{0}\right)$ such that for any nowhere zero local section $s: U \rightarrow E^{0}$ in the vector bundle $E$

$$
\alpha(s)=s^{*} \alpha .
$$

Vice versa, if $\alpha \in \Omega^{1}\left(E^{0}\right)$ is a connection 1-form then there is a unique connection $\nabla$ in $E$ such that (30) holds for any section $s: U \rightarrow E^{0}$ and any open set $U \subset M$. This accounts for the pingpong between the theory of connections in vector bundles and the theory of connections in principal bundles that is so well-described in the classical monograph [56]. However, our presentation here follows [27] (and the result we just quoted is Proposition 1.5.1 in [27], p. 101). To our knowledge, B. Kostant never wrote the second part of [27] (despite his promise in the introduction to [27]). 
Let $(E, \nabla)$ be a complex line bundle with connection and let $\alpha \in \Omega^{1}\left(E^{0}\right)$ be the corresponding connection 1-form. There is a unique closed 2-form $\Omega \in \Omega^{2}(M)$ such that

$$
d \alpha=\pi_{0}^{*} \Omega
$$

where $\pi_{0}: E^{0} \rightarrow M$ is the projection (i.e., $\pi_{0}=\left.\pi\right|_{E^{0}}$ ). Cf. Proposition 1.6.1 in [27], p. 103. The 2-form in (31) is the curvature of $(E, \alpha)$, and we adopt the notation

$$
\Omega=\operatorname{curv}(E, \nabla) .
$$

We give a few details of the proof in order to give the reader a quantitative glimpse of what $\Omega$ looks like. Toward this end we adopt the following notations. Let $N$ be a $C^{\infty}$ manifold and let

$$
\Pi_{1}:(\mathbb{C} \backslash\{0\}) \times N \rightarrow \mathbb{C} \backslash\{0\}, \quad \Pi_{2}:(\mathbb{C} \backslash\{0\}) \times N \rightarrow N
$$

be the canonical projections of the product manifold $(\mathbb{C} \backslash\{0\}) \times N$. To any given $C^{\infty}$ map $f: N \rightarrow E^{0}$ we associate the map

$$
\begin{gathered}
\sigma_{f}:(\mathbb{C} \backslash\{0\}) \times N \rightarrow E^{0}, \quad \sigma_{f}(\lambda, x)=\lambda f(x), \\
\lambda \in \mathbb{C} \backslash\{0\}, \quad x \in N .
\end{gathered}
$$

If $\alpha \in \Omega^{1}\left(E^{0}\right)$ is a connection 1 -form then

$$
\sigma_{f}^{*} \alpha=\Pi_{1}^{*}\left(\frac{1}{2 \pi i} \frac{1}{z} d z\right)+\Pi_{2}^{*}\left(f^{*} \alpha\right) .
$$

Cf. Lemma 1.5.1 in [27], p. 100. Moreover let $g: N \rightarrow E^{0}$ be another $C^{\infty}$ map such that $\pi^{0} \circ f=\pi^{0} \circ g$ (where $\pi^{0}: E^{0} \rightarrow M$ is the projection). Then, for every $x \in N$ the vectors $f(x)$ and $g(x)$ lie in the same fibre of $E^{0}$, hence the function

$$
\phi=\frac{g}{f}: N \rightarrow \mathbb{C}, \quad \phi(x)=\frac{g(x)}{f(x)}, \quad x \in N,
$$

is well defined. By a result in [27] (cf. (1.5.3) in [27], p. 101)

$$
g^{*} \alpha=f^{*} \alpha+\frac{1}{2 \pi i} \frac{1}{\phi} d \phi
$$

To build $\Omega \in \Omega^{2}(M)$ as in (31) let $s: U \rightarrow E^{0}$ be a nowhere zero local section in $E$ and let us apply Formula (32) with $N=U$ and $f=s$. That is, the map $\sigma_{s}:(\mathbb{C} \backslash\{0\}) \times U \rightarrow E^{0}$ pulls back the connection 1-form $\alpha \in \Omega^{1}\left(E^{0}\right)$ to the 1-form

$$
\begin{gathered}
\sigma_{s}^{*} \alpha=\Pi_{1}^{*}\left(\frac{1}{2 \pi i} \frac{1}{z} d z\right)+\Pi_{2}^{*}\left(s^{*} \alpha\right)=\text { (by (30)) } \\
=\Pi_{1}^{*}\left(\frac{1}{2 \pi i} \frac{1}{z} d z\right)+\Pi_{2}^{*} \alpha(s)
\end{gathered}
$$

hence (as exterior differentiation commutes with pullback by a $C^{1}$ map)

$$
\sigma_{s}^{*}(d \alpha)=\Pi_{2}^{*} d \alpha(s) .
$$

Moreover, if $r: U \rightarrow E^{0}$ is another nonzero section then (by (33) with $g=r$ and $f=s$ and $\phi=r / s)$

$$
r^{*} \alpha=s^{*} \alpha+\frac{1}{2 \pi i} \frac{1}{\phi} d \phi
$$


or

$$
\alpha(r)=\alpha(s)+\frac{1}{2 \pi i} \frac{1}{\phi} d \phi
$$

hence (by $\left.d\left(\phi^{-1} d \phi\right)=0\right)$

$$
d \alpha(r)=d \alpha(s)
$$

In particular, if $s_{\beta}(z)=\Phi_{\beta}^{-1}(z, 1)$ for any $z \in U_{\beta}$ and any $\beta \in J$ then

$$
d \alpha\left(s_{\beta}\right)=d \alpha\left(s_{\gamma}\right) \text { on } U_{\beta} \cap U_{\gamma}
$$

Hence, there is a unique 2 -form $\Omega \in \Omega^{2}(M)$ such that

$$
j_{\beta}^{*} \Omega=d \alpha\left(s_{\beta}\right) \text { on } U_{\beta}, \quad \beta \in J \text {, }
$$

where $j_{\beta}: U_{\beta} \hookrightarrow M$ is the inclusion. On the other hand, let us observe that the diagram

$$
\begin{array}{cll}
(\mathbb{C} \backslash\{0\}) \times U_{\beta} & \stackrel{\sigma_{s}}{\longrightarrow} & E^{0} \\
\Pi_{2} \downarrow & \downarrow \pi_{0} \\
U_{\beta} & \stackrel{j_{\beta}}{\longrightarrow} & M
\end{array}
$$

is commutative. Then (by (34))

$$
\begin{gathered}
\sigma_{s_{\beta}}^{*}(d \alpha)=\Pi_{2}^{*} d \alpha\left(s_{\beta}\right)=\Pi_{2}^{*} j_{\beta}^{*} \Omega= \\
=\left(j_{\beta} \circ \Pi_{2}\right)^{*} \Omega=\left(\pi_{0} \circ \sigma_{s_{\beta}}\right)^{*} \Omega=\sigma_{s_{\beta}}^{*} \pi_{0}^{*} \Omega
\end{gathered}
$$

and $\sigma_{s_{\beta}}$ is injective, hence $d \alpha=\pi_{0}^{*} \Omega$ on $\pi_{0}^{-1}\left(U_{\beta}\right)$ for every $\beta \in J$, thus yielding (31). Of course, it is Formula (35) (giving an explicit local representation of $\Omega$ ), rather than (31), that sheds light on how $\Omega$ is really built.

\subsection{Canonical Hermitian Connection}

Let $\nabla$ be a connection in $E$ and $\alpha \in \Omega^{1}\left(E^{0}\right)$ its connection 1-form. A Hermitian bundle metric $H$ on $E$ is $\alpha$-invariant if $X(H(s, r))=H\left(\nabla_{X} s, r\right)+H\left(s, \nabla_{X} r\right)$ for any $X \in \mathfrak{X}(M)$ and any $s, r \in \Omega^{0}(E)$. For every pair $(E, \nabla)$ the following statements are equivalent:

(i) There is an $\alpha$-invariant Hermitian bundle metric $H$ on $E$.

(ii) The real differential 1-form $2 \pi i(\alpha-\bar{\alpha}) \in \Omega^{1}\left(E^{0}\right)$ is exact.

Cf. Proposition 1.9.1 in [27], p. 109. If this is the case, then $H$ is uniquely determined up to a positive constant and

$$
2 \pi i(\alpha-\bar{\alpha})=d \log |H|^{2}
$$

where $|H|^{2}: E^{0} \rightarrow(0,+\infty)$ is the $C^{\infty}$ function given by $|H|^{2}(v)=H(v, v)$ for any $v \in E^{0}$. Cf. (1.9.2) in [27], p. 110. Additionally, if $H$ is an $\alpha$-invariant Hermitian bundle metric on $E$, then (again by a result in [27], p. 111) $\Omega=\operatorname{curv}(E, \nabla) \in \Omega^{2}(M)$ is a real 2-form.

Given a connection $\nabla$ in $E$ and $s \in \Omega^{0}(E)$ let $\nabla^{0,1} s$ denote the restriction of $\nabla s$ to $C^{\infty}\left(T^{0,1}(M)\right)$, where $T^{1,0}(M) \rightarrow M$ is the holomorphic tangent bundle (i.e., the portion of $T^{1,0}(M)$ over $U_{\beta}$ is the span of $\left\{\partial / \partial z_{\beta}^{j}: 1 \leq j \leq n\right\}$ for any $\left.\beta \in J\right)$ and $T^{0,1}(M)=\overline{T^{1,0}(M)}$. Given a holomorphic line bundle $E$ with a Hermitian bundle metric $H$ there is a unique connection $\nabla$ in $E$ such that (1) $\nabla^{0,1}=\bar{\partial}_{E}$ and (2) $H$ is $\alpha$-invariant. The result is due to S. Nakano (cf. [72]) and said $\nabla$ is the canonical Hermitian 
connection of $(E, H)$. Cf. also [56], Vol. II, p. 183. For any fixed $\beta \in J$, one has $\bar{\partial}_{E} s_{\beta}=0$, and hence the canonical Hermitian connection of $(E, H)$ is locally represented as (by (30) and (36))

$$
\nabla_{Z} s_{\beta}=\frac{1}{2 \pi i} Z\left(\log H_{\beta \bar{\beta}}\right) s_{\beta}, \quad \nabla_{\bar{Z}} s_{\beta}=0,
$$

for any $Z \in C^{\infty}\left(T^{1,0}(M)\right)$, where $H_{\beta \bar{\beta}}=H\left(s_{\beta}, s_{\beta}\right)=\gamma_{\beta}\left(\right.$ or $\left.H_{\beta \bar{\beta}}=|H|^{2}\left(s_{\beta}\right)\right)$.

\subsection{Liouville Measure}

Following A. Odzijewicz's work [9], we endow $(E, H)$ with the canonical Hermitian connection $\nabla$ and assume that $\Omega=\operatorname{curv}(E, \nabla)$ is nondegenerate, so that $(M, \Omega)$ is a symplectic manifold. With that assumption in mind, let us set

$$
\Omega_{j \bar{k}}^{\alpha}=\Omega\left(\partial / \partial z_{\alpha}^{k}, \partial / \partial \bar{z}_{\alpha}^{k}\right), \quad \Omega_{\alpha \bar{\alpha}}=\operatorname{det}\left[\Omega_{j \bar{k}}^{\alpha}\right],
$$

so that

$$
\Omega_{\beta \bar{\beta}}=\left|\frac{\partial z_{\beta}}{\partial z_{\alpha}}\right|^{2} \Omega_{\alpha \bar{\alpha}}
$$

on $U_{\alpha} \cap U_{\beta}$. The Liouville form is the $2 n$-form $\Omega^{n}=\Omega \wedge \cdots \wedge \Omega$ ( $n$ terms). Locally on $U_{\alpha}$

$$
\Omega^{n}=2^{n} i^{n(n-1)} \Omega_{\alpha \bar{\alpha}} d z_{\alpha}^{1} \wedge \cdots \wedge d z_{\alpha}^{n} \wedge d \bar{z}_{\alpha}^{1} \wedge \cdots \wedge d \bar{z}_{\alpha}^{n} .
$$

There is yet another Hermitian metric $\tilde{H}$ of customary use on $E$, locally given by

$$
\tilde{H}\left(s_{\alpha}, s_{\alpha}\right)_{z}=(-i)^{n} \frac{\Omega_{\alpha \bar{\alpha}}(z)}{K_{\alpha \bar{\alpha}}(z, z)}
$$

for any $z \in U_{\alpha}$. Here (by the previous nondegeneracy assumption) $\Omega_{\alpha \bar{\alpha}} \neq 0$ everywhere in $U_{\alpha}$ and one tacitly assumes that the right hand side of (38) is $>0$ (otherwise one replaces $\tilde{H}$ by $-\tilde{H}$ ). By (24) for $\gamma=\beta, \delta=\alpha$ and $\zeta=z$

$$
K_{\beta \bar{\beta}}(z, z)=\left|\frac{\partial \zeta_{\alpha}}{\partial \zeta_{\beta}}(z)\right|^{2}\left|g_{\alpha \beta}(z)\right|^{2} K_{\alpha \bar{\alpha}}(z, z)
$$

yielding (together with (37))

$$
\frac{K_{\beta \bar{\beta}}(z, z)}{\Omega_{\alpha \bar{\alpha}}(z)}=\left|g_{\alpha \beta}(z)\right|^{2} \frac{K_{\alpha \bar{\alpha}}(z, z)}{\Omega_{\alpha \bar{\alpha}}(z)}
$$

so that $\tilde{H}$ is globally defined. Geometric quantization theory commonly takes $\mathcal{M}$ to be the space of square integrable holomorphic sections in $E$, with respect to the Liouville measure (locally given by)

$$
d \mu_{L}=(-i)^{n} \Omega_{\alpha \bar{\alpha}} d \zeta_{\alpha}^{1} \wedge \cdots \wedge d \zeta_{\alpha}^{n} \wedge d \bar{\zeta}_{\alpha}^{1} \wedge \cdots \wedge d \bar{\zeta}_{\alpha}^{n}
$$

on $U_{\alpha}$. The two approaches may be made to merge by a clever choice of (local) weights of integration—-that is, let us assume (together with A. Odzijewicz, [9], p. 584) that

$$
\tilde{H}=C H
$$

for some constant $C>0$, so that the local expression of the Liouville measure becomes

$$
d \mu_{L}(\zeta)=C K_{\alpha \bar{\alpha}}(\zeta, \zeta) \gamma_{\alpha}(\zeta) d \zeta_{\alpha}^{1} \wedge \cdots \wedge d \zeta_{\alpha}^{n} \wedge d \bar{\zeta}_{\alpha}^{1} \wedge \cdots \wedge d \bar{\zeta}_{\alpha}^{n} .
$$


Next, starting from

$$
\begin{gathered}
\alpha_{\beta}(Z)=\frac{1}{2 \pi i} Z\left(\log \gamma_{\beta}\right), \quad \alpha_{\beta}(\bar{Z})=0, \quad Z \in T^{1,0}(M), \\
j_{\beta}^{*} \Omega=d s_{\beta}, \quad \beta \in J,
\end{gathered}
$$

one has

$$
\begin{gathered}
\Omega_{j \bar{k}}^{\beta}=\Omega\left(\frac{\partial}{\partial z_{\beta}^{j}}, \frac{\partial}{\partial \bar{z}_{\beta}^{k}}\right)=\left(d s_{\beta}\right)\left(\frac{\partial}{\partial z_{\beta}^{j}}, \frac{\partial}{\partial \bar{z}_{\beta}^{k}}\right)= \\
=-\frac{1}{2} \frac{\partial}{\partial \bar{z}_{\beta}^{k}}\left(\alpha_{\beta}\left(\frac{\partial}{\partial z_{\beta}^{j}}\right)\right)=-\frac{1}{4 \pi i} \frac{\partial^{2} \log \gamma_{\beta}}{\partial z_{\beta}^{j} \partial \bar{z}_{\beta}^{k}}
\end{gathered}
$$

and Odzijewicz's structural assumption (39) becomes

$$
\operatorname{det}\left[\frac{\partial^{2} \log \gamma_{\beta}}{\partial z_{\beta}^{j} \partial \bar{z}_{\beta}^{k}}(z)\right]=C(4 \pi)^{n} K_{\beta \bar{\beta}}(z, z) \gamma_{\beta}(z)
$$

for any $z \in U_{\beta}$.

\subsection{Transition Probability Amplitudes}

Let $z \in M$ be a classical state and let $\mathcal{K}(z)=\left[k_{z, \bar{\alpha}}\right] \in \mathbb{C P}(\mathcal{M})$ be the corresponding coherent state. Given the classical states $z \in U_{\alpha}$ and $\zeta \in U_{\beta}$, thought of as identified with the coherent states $\mathcal{K}(z)$ and $\mathcal{K}(\zeta)$, the transition probability amplitude from $z$ to $\zeta$ is

$$
a_{\beta \bar{\alpha}}(\zeta, z)=\left\langle\frac{k_{z, \bar{\alpha}}}{\left\|k_{z, \bar{\alpha}}\right\|}, \frac{k_{\zeta, \bar{\beta}}}{\left\|k_{\zeta, \bar{\beta}}\right\|}\right\rangle
$$

and $\left|a_{\beta \bar{\alpha}}(\zeta, z)\right|^{2}$ is the transition probability density. Then

$$
a_{\beta \bar{\alpha}}(\zeta, z)=\frac{K_{\beta \bar{\alpha}}(\zeta, z)}{K_{\alpha \bar{\alpha}}(z, z)^{1 / 2} K_{\beta \bar{\beta}}(\zeta, \zeta)^{1 / 2}}
$$

so that

$$
a_{\delta \bar{\gamma}}(\zeta, z)=\frac{g_{\mu \delta}(\zeta)}{\left|g_{\mu \delta}(\zeta)\right|} \frac{\frac{\partial \zeta_{\mu}}{\partial \zeta_{\delta}}(\zeta)}{\left|\frac{\partial \zeta_{\mu}}{\partial \zeta_{\delta}}(\zeta)\right|} \frac{g_{\sigma \gamma}(z)}{\left|g_{\sigma \gamma}(z)\right|} \frac{\frac{\partial \zeta_{\sigma}}{\partial \zeta_{\gamma}}(z)}{\left|\frac{\partial \zeta_{\sigma}}{\partial \zeta_{\gamma}}(z)\right|} a_{\mu \bar{\sigma}}(\zeta, z) .
$$

That is, under a transformation of local frames $a_{\beta \bar{\alpha}}(\zeta, z)$ changes by a phase factor $e^{i \phi}$ of global phase

$$
\phi=\arg \left[g_{\mu \delta}(\zeta) g_{\sigma \gamma}(z) \frac{\partial \zeta_{\mu}}{\partial \zeta_{\delta}}(\zeta) \frac{\partial \zeta_{\sigma}}{\partial \zeta_{\gamma}}(z)\right]
$$

In particular $\left|a_{\delta \bar{\gamma}}(\zeta, z)\right|=\left|a_{\beta \bar{\alpha}}(\zeta, z)\right|$ so the transition probability density does not depend upon the choice of local frames, both on $E$ and $T^{1,0}(M)$. Of course, it depends on the states $z \in U_{\alpha}$ and $\zeta \in U_{\beta}$.

The transition probability amplitude from $z$ to $\zeta$ with simultaneous transition through $w \in U_{\gamma}$ is

$$
a_{\gamma \bar{\alpha}}(w, z) a_{\beta \bar{\gamma}}(\zeta, w)
$$


It does not depend upon the choice of $\gamma \in J$ such that $w \in U_{\gamma}$. Let $\left\{\chi_{\alpha}\right\}_{\alpha \in J}$ be a $C^{\infty}$ partition of unity subordinated to the open cover $\left\{U_{\alpha}\right\}_{\alpha \in J}$, so that $\operatorname{Supp}\left(\chi_{\alpha}\right) \subset U_{\alpha}$ and $\sum_{\alpha \in I} \chi_{\alpha}=1$ on $M$. We start from (23)

$$
\begin{gathered}
K_{\beta \bar{\alpha}}(\zeta, z)=i^{n^{2}} \sum_{\gamma \in J} \int_{U_{\gamma}} \chi_{\gamma} H^{*}\left(k_{z, \bar{\alpha}}, k_{\zeta, \bar{\beta}}\right)= \\
=i^{n^{2}} \sum_{\gamma \in J} \int_{U_{\gamma}} \chi_{\gamma} K_{\gamma \bar{\alpha}}(w, z) K_{\beta \bar{\gamma}}(\zeta, w) H_{\gamma \bar{\gamma}}(w) d w_{\gamma}^{1 \cdots n} \wedge d \bar{w}_{\gamma}^{1 \cdots n}
\end{gathered}
$$

where we have set

$$
d w_{\gamma}^{1 \cdots n}=d w_{\gamma}^{1} \wedge \cdots \wedge d w_{\gamma}^{n}, \quad d \bar{w}_{\gamma}^{1 \cdots n}=\overline{d w_{\gamma}^{1 \cdots n}} .
$$

Then, multiplication by $1 /\left[K_{\alpha \bar{\alpha}}(z, z)^{1 / 2} K_{\beta \bar{\beta}}(\zeta, \zeta)^{1 / 2}\right]$ leads to

$$
\begin{aligned}
& a_{\beta \bar{\alpha}}(\zeta, z)=i^{n^{2}} \sum_{\gamma \in J} \int_{U_{\gamma}} a_{\gamma \bar{\alpha}}(w, z) a_{\beta \bar{\gamma}}(\zeta, w) \times \\
& \times \chi_{\gamma}(w) K_{\gamma \bar{\gamma}}(w, w) H_{\gamma \bar{\gamma}}(w) d w_{\gamma}^{1 \cdots n} \wedge d \bar{w}_{\gamma}^{1 \cdots n}
\end{aligned}
$$

or (by (39))

$$
a_{\beta \bar{\alpha}}(\zeta, z)=i^{n^{2}} \sum_{\gamma \in J} \int_{U_{\gamma}} \chi_{\gamma}(w) a_{\gamma \bar{\alpha}}(w, z) a_{\beta \bar{\gamma}}(\zeta, w) d \mu_{L}(w)
$$

\subsection{Parallel Translation and Transition Probability Amplitudes}

Let $(E, \nabla)$ be a complex line bundle with connection. Let $I \subset \mathbb{R}$ be an interval and let $\Gamma: I \rightarrow U$ be a $C^{1}$ curve lying in the open set $U \subset M$ such that $C^{\infty}$ sections $s: U \rightarrow E^{0}$ exist. A map $r: I \rightarrow E$ is a section along $\Gamma$ if $r(t) \in E_{\Gamma(t)}$ for every $t \in I$. If $s: U \rightarrow E^{0}$ is an arbitrary nowhere vanishing section then the function $r /(s \circ \Gamma): I \rightarrow \mathbb{C}$ is well defined-that is,

$$
\left(\frac{r}{s \circ \Gamma}\right)(t)=\frac{r(t)}{s(\Gamma(t))}
$$

for any $t \in I$. The covariant derivative of $r: I \rightarrow E$ is the section $\nabla r: I \rightarrow E$ along $\Gamma$ such that

$$
\left(\frac{\nabla r}{s \circ \Gamma}\right)(t)=\left(\frac{r}{s \circ \Gamma}\right)^{\prime}(t)+\frac{1}{2 \pi i}\left(\frac{r}{s \circ \Gamma}\right)(t) \alpha(s)_{\Gamma(t)} \dot{\Gamma}(t)
$$

for every section $s: U \rightarrow E^{0}$ and any $t \in I$. Here $\dot{\Gamma}(t)=(d \Gamma / d t)(t)$. Additionally, if $\varphi: I \rightarrow \mathbb{C}$ is a smooth function then $\varphi^{\prime}=d \varphi / d t$. If $r=s \circ \Gamma$ then $(\nabla r)(t)=\left(\nabla_{\dot{\Gamma}} s\right)_{\Gamma(t)}$. We exploit these notions to discuss parallel displacement along a curve in $M$ with respect to the canonical Hermitian connection $\nabla^{K}$ on the holomorphic line bundle $\Lambda^{n, 0}(M) \otimes E \rightarrow M$ endowed with the Hermitian bundle metric

$$
\begin{gathered}
H^{K}\left(s_{\gamma} \otimes d z_{\gamma}^{1 \cdots n}, s_{\gamma} \otimes d z_{\gamma}^{1 \cdots n}\right)_{z}=\frac{1}{F_{\gamma \bar{\gamma}}}, \\
d z_{\gamma}^{1 \cdots n}=d z_{\gamma}^{1} \wedge \cdots \wedge d z_{\gamma}^{n}, \quad F_{\gamma \bar{\gamma}}(z)=K_{\gamma \bar{\gamma}}(z, z), \quad z \in U_{\gamma}, \quad \gamma \in J .
\end{gathered}
$$

We shall work with the unitary frame $\sigma_{\gamma}=F_{\gamma \bar{\gamma}}^{1 / 2} s_{\gamma} \otimes d z_{\gamma}^{1 \cdots n}$ so that $H^{K}\left(\sigma_{\gamma}, \sigma_{\gamma}\right)=1$. As $\Lambda^{n, 0}(M) \otimes$ $E$ is holomorphic, it possesses a $\bar{\partial}$ operator

$$
\bar{\partial}_{\Lambda^{n, 0} \otimes E}: \Omega^{n, 0}(E) \rightarrow C^{\infty}\left(T^{0,1}(M)^{*} \otimes \Lambda^{n, 0}(M) \otimes E\right)
$$


such that $\bar{\partial}_{\Lambda^{n, 0} \otimes E}\left(s_{\gamma} \otimes d z_{\gamma}^{1 \cdots n}\right)=0$. Let $\alpha^{K}(\sigma) \in \Omega^{1}(U)$ be defined by

$$
\begin{gathered}
\alpha^{K}(\sigma) X=\frac{1}{2 \pi i} \frac{\nabla_{X}^{K} \sigma}{\sigma}, \\
X \in \mathfrak{X}(M), \quad \sigma \in \Omega^{0}\left(\Lambda^{n, 0}(M) \otimes E\right) .
\end{gathered}
$$

Then

$$
\left(\nabla^{K}\right)^{0,1}=\bar{\partial}_{\Lambda^{n, 0} \otimes E}
$$

together with that $\alpha^{K}\left(\sigma_{\gamma}\right)$-invariance of $H^{K}$ yield

$$
\alpha^{K}\left(\sigma_{\gamma}\right) Z=-\frac{1}{4 \pi i} Z\left(\log F_{\gamma \bar{\gamma}}\right), \quad Z \in T^{1,0}(M)
$$

Let $\Gamma:[0,1] \rightarrow M$ be a piecewise $C^{1}$ curve joining $z \in U_{\alpha}$ and $\zeta \in U_{\beta}$, that is, $\Gamma(0)=z$ and $\Gamma(1)=\zeta$. We may choose $\gamma_{j} \in J, 1 \leq j \leq N$, and a partition $\tau_{1}=0<\tau_{2}<\cdots<\tau_{N-1}<1=\tau_{N}$ such that

$$
\begin{gathered}
\gamma_{1}=\alpha, \quad \gamma_{N}=\beta, \quad \Gamma_{j}(t) \in U_{\gamma_{j}}, \\
\Gamma_{j}(t)=\Gamma(t), \quad \tau_{j} \leq t \leq \tau_{j+1}, \quad 1 \leq j \leq N-1 .
\end{gathered}
$$

To build the parallel displacement operator

$$
P_{\Gamma}:\left(\Lambda^{n, 0}(M) \otimes\right)_{\Gamma(0)} \rightarrow\left(\Lambda^{n, 0}(M) \otimes\right)_{\Gamma(1)}
$$

one solves the ODE

$$
\begin{gathered}
f_{j}^{\prime}(t)+2 \pi i f_{j}(t) \alpha^{K}\left(\sigma_{\gamma_{j}}\right)_{\Gamma(t)} \dot{\Gamma}(t)=0, \\
f_{j}=\frac{r_{j}}{\sigma_{\gamma_{j}} \circ \Gamma}, \quad r_{j}:\left[\tau_{j}, \tau_{j+1}\right] \rightarrow \Lambda^{n, 0}(M) \otimes E
\end{gathered}
$$

with the initial condition

$$
r_{1}(0)=\omega_{0} \in\left(\Lambda^{n, 0}(M) \otimes E\right)_{z}, \quad r_{j}\left(\tau_{j}\right)=r_{j-1}\left(\tau_{j}\right), \quad 2 \leq j \leq N-1 .
$$

The solution to (43) is

$$
f_{j}(t)=C_{j} \exp \left\{-2 \pi i \int_{\tau_{j}}^{t} \alpha^{K}\left(\sigma_{\gamma_{j}}\right)_{\Gamma(\tau)} \dot{\Gamma}(\tau) d \tau\right\}
$$

where $C_{j} \in \mathbb{C}$ is a constant of integration. Additionally,

$$
\alpha\left(\sigma_{\gamma_{j}}\right)_{\Gamma(t)} \dot{\Gamma}(t)=\frac{1}{2 \pi} \operatorname{Im}\left(\bar{\partial} \log F_{\gamma_{j} \bar{\gamma}_{j}}\right)_{\Gamma(t)}
$$

and of course $P_{\Gamma}\left(\omega_{0}\right)=r_{\omega_{0}}(1)$ where $r_{\omega_{0}}(t)=r_{j}(t)$ for any $\tau_{j} \leq t<\tau_{j+1}$. Next, let us set $z_{j}=\Gamma\left(\tau_{j}\right)$, $1 \leq j \leq N$. The transition probability amplitude from state $z$ to state $\zeta$ with simultaneous transition through the states $z_{2}, \cdots, z_{N-1}$ is $\prod_{j=1}^{N-1} a_{\alpha_{j+1}} \bar{\alpha}_{j}\left(z_{j+1}, z_{j}\right)$. If $\tau_{j}=(j-1) / N, 1 \leq j \leq N$, then

$$
a_{\beta \bar{\alpha}}(\Gamma ; \zeta, z)=\lim _{N \rightarrow \infty} \exp \sum_{j=1}^{N-1} \log a_{\gamma_{j+1} \bar{\gamma}_{j}}\left(z_{j+1}, z_{j}\right)
$$

is the transition probability amplitude along $\Gamma$. By a result in [9], p. $585, a_{\beta \bar{\alpha}}(\Gamma ; \zeta, z)$ is the parallel displacement with respect to $\nabla^{K}$, from $z$ to $\zeta$ along $\Gamma$. 
Following the scheme proposed in [10], M. Horowski and A. Odzijewicz (cf. [4]) study the Kepler problem in full detail. According to I.M. Mladenov (cf. [73]) this confirms the power of the theory developed by A. Odzijewicz over the years (cf. [3,8-10]) and calls for further developments and applications. Another such application (of the matters in [8], described by us at a certain length in the present survey) is due to A. Odzijewicz and M. Swietochowski (cf. [12]) and is devoted to the study of the MIC-Kepler problem, viewed as a reduction of the harmonic oscillator system defined on the twistor space.

Cf. also D. Beltiţă and J.E. Galé [74], R. Coquereaux and A. Jadczyk [75], M. Englis [76], V.V. Kisil [77], M.I. Monastyrskii and A.M. Perelomov [78].

\subsection{Complex Orbifolds}

The problem of building a quantization theory for mechanical systems whose phase space is a complex orbifold is open. Cf. J. Masamune et al. [79] for the main properties of complex and CR orbifolds (cf. also E. Barletta et al. [80], p. 201-219). Space-time orbifolds do occur in general relativity and gravitation theory (cf. V. Balasubramanian et al. [81]). For example, orbifolds $O$ whose local geometry is that of an anti-de Sitter space-time have been demonstrated by a number of authors (cf. K. Berndt and D. Lust [82], J. Son [83], E.J. Martinec and W. McElgin [84]) and the (total space of the) cotangent bundle $M=T^{*}(O)$ is an orbifold (cf. I. Satake [85]). The development of orbifold theory has known a period of stagnation due to the lack of an appropriate notion of a "map of orbifolds", yet the difficulty seems to have been overcome (cf. Y. Takeuchi [86], M. Yamasaki [87], I. Moderduk and D.A. Pronk [88], W-M. Chen [89]). Several leading quantized classical systems have complex manifolds as phase spaces; for example, the space of orbits of the $n$-dimensional harmonic isotropic oscillator is $\mathbb{C P}^{n-1}$ (cf. N. Hurt [90]), the phase space of a spin system is $\mathbb{C P}^{1}$, and $\mathbb{C P}^{1} \times \mathbb{C P}^{1}$ is the phase space of orbits corresponding to the negative energy level in the Kepler problem (cf. D.J. Simms [91]). The role of weighted projective spaces as (compact) orbifolds is exhibited by A.B. Aazami, A.O. Petters, and J.M. Rabin [92] (cf. also A.B. Azami, C.R. Keeton, and A.O. Peters [93], as well as A.O. Peters and M.C. Werner [94]).

\subsection{Regular Quantization}

In the present section we briefly report on works by A. Loi (cf. [95]) and C. Arezzo and A. Loi (cf. $[96,97]$ ) within Kähler geometry, as related to quantization of dynamical systems whose phase space is a complex manifold.

The story starts with G. Tian's solution (cf. [98]) to Yau's conjecture that polarized Kähler metrics on a compact complex manifold $M$ can be obtained as the limit of (a sequence of) Bergmann metrics. A remarkable ingredient in Tian's work (cf. op. cit.) is the so-called Epsilon function $\epsilon_{m}: M \rightarrow[0,+\infty)$ (with $m \in \mathbb{Z}_{+}$) appearing independently in M. Cahen, S. Gutt, and J.H. Rawnsley's geometric interpretation (cf. [99]) of F.A. Berezin's (cf. [100]) quantization procedure (itself including the familiar Wick and Weyl quantizations, cf. [101]). An asymptotic expansion (now known as the Tian-Yau-Zelditch expansion) in the variable $m$ of $\epsilon_{m}(x)$ was produced by S. Zelditch (cf. [102]) and subsequently Z. Lu computed (cf. [103]) the first three terms of the expansion.

The first to have observed that Tian's function and the Chen-Gutt-Rawnsley Epsilon function $\epsilon_{m}(x)$ coincide (up to a multiplicative factor $m^{n}$, where $n$ is the complex dimension of $M$ ) appear to be C. Arezzo and A. Loi (cf. [96]). Arezzo-Loi's general purposes go towards classifying Kähler manifolds admitting a regular quantization (i.e., one for which $\epsilon_{m}(x)$ is constant for every $m \in \mathbb{Z}_{+}$) and understanding when finite Tian-Yau-Zelditch expansions might occur. 


\section{Djrbashian Kernels on Siegel Domains}

\subsection{Djrbashian-Karapetyan Projection}

Let us start with

$$
\alpha \in \mathbb{R}, \quad \alpha>-1, \quad \beta \in \mathbb{C}, \quad \operatorname{Re}(\beta)>\frac{\alpha-1}{2},
$$

and consider the linear operator

$$
\begin{gathered}
\left(T_{\beta} f\right)(w)=2^{n-1+\beta} c_{n, \beta} \int_{\Omega_{n}} \frac{f(\zeta)\left(\operatorname{Im}\left(\zeta_{1}\right)-\left|\zeta^{\prime}\right|^{2}\right)^{\beta} d \mu(\zeta)}{\left[i\left(\bar{\zeta}_{1}-w_{1}\right)-2\left\langle w^{\prime}, \zeta^{\prime}\right\rangle\right]^{n+1+\beta}}, \\
f \in L_{\alpha}^{2}\left(\Omega_{n}\right)=L^{2}\left(\Omega_{n}, \gamma_{\alpha}\right), \quad w \in \Omega_{n}, \\
c_{n, \beta}=\pi^{-n}(\beta+1) \cdots(\beta+n) .
\end{gathered}
$$

Cf. (2.15) in [36], p. 98. By Theorems 2.1 and 3.1 in [36], $T_{\beta}$ is a continuous linear operator from $L_{\alpha}^{2}\left(\Omega_{n}\right)$ to $H_{\alpha}^{2}\left(\Omega_{n}\right)=L^{2} H\left(\Omega_{n}, \gamma_{\alpha}\right)$, referred to in [28] as the Djrbashian-Karapeyan projection. If

$$
h_{z}(\zeta)=2^{n-1+\bar{\beta}} \frac{\overline{c_{n, \beta}}\left[\operatorname{Im}\left(\zeta_{1}\right)-\left|\zeta^{\prime}\right|^{2}\right]^{\bar{\beta}-\alpha}}{\left[i\left(\bar{z}_{1}-\zeta_{1}\right)-2\left\langle\zeta^{\prime}, z^{\prime}\right\rangle\right]^{n+1+\beta}}, \quad z, \zeta \in \Omega_{n},
$$

then

$$
h_{z} \in L^{2}\left(\Omega_{n}, \gamma_{\alpha}\right), \quad z \in \Omega_{n} .
$$

This is Lemma 1 in [28], p. 49, and the proof relies on a result by R.R. Coifman and R. Rochberg (cf. Lemma 2.2 in [104]).

\subsection{Saitoh's Construction and Djrbashian Kernels}

S. Saitoh has devised (cf. [105]) a very general method for organizing the range of a linear operator (induced by a Hilbert space valued function) as an RKH space. We recall the essentials of Saitoh's result and apply it to the Djrbashian-Karapetyan projection. Given a set $E \neq \varnothing$, a Hilbert space $\mathcal{H}$, and a function $h: E \rightarrow \mathcal{H}$, let us consider the linear map

$$
L: \mathcal{H} \rightarrow \mathcal{F}(E), \quad(L F)(p)=(F, h(p))_{\mathcal{H}}, \quad F \in \mathcal{H}, \quad p \in E .
$$

By a result of S. Saitoh (cf. Theorem 2.1 in [105], p. 75) the range $\mathcal{R}(L)$ of $L$ may be organized as an RKH space with the inner product

$$
\begin{gathered}
(f, g)_{\mathcal{R}(L)}=(P F, P G)_{\mathcal{H}}, \\
f, g \in \mathcal{R}(L), \quad F \in L^{-1}(f), \quad G \in L^{-1}(g),
\end{gathered}
$$

where $P: \mathcal{H} \rightarrow \mathcal{H} \ominus \mathcal{N}(L)$ is the natural projection and $\mathcal{N}(L)$ is the null space of $L$. Then

$$
\|f\|_{\mathcal{R}(L)}=\inf \left\{\|F\|_{\mathcal{H}}: F \in L^{-1}(f)\right\}
$$

and

$$
K(p, q)=(h(p), h(q))_{\mathcal{H}}, \quad p, q \in E,
$$

is a reproducing kernel for $\mathcal{R}(L)$. Additionally, $L$ is an isometry of $\mathcal{H}$ onto $\mathcal{R}(L)$ if and only if the system $\{h(p): p \in E\}$ is complete in $\mathcal{H}$. Cf. also [106], p. 51. Going back to the Djrbashian-Karapetyan projection, let us set

$$
K_{\beta}(\zeta, z)=\left(h_{z}, h_{\zeta}\right)_{\gamma_{\alpha}}, \quad \zeta, z \in \Omega_{n}
$$


Then, $K_{\beta}$ is well-defined as a consequence of (45). Let

$$
P_{\beta}: L_{\alpha}^{2}\left(\Omega_{n}\right) \rightarrow L_{\alpha}^{2}\left(\Omega_{n}\right) \ominus \mathcal{N}\left(T_{\beta}\right)
$$

be the orthogonal projection and note that $P_{\beta} h_{z}=h_{z}$ for any $z \in \Omega_{n}$. As

$$
\left(T_{\beta} f\right)(\zeta)=\left(f, h_{\zeta}\right)_{\gamma_{\alpha}}, \quad f \in L_{\alpha}^{2}\left(\Omega_{n}\right), \quad \zeta \in \Omega_{n},
$$

it follows that

(i) $K_{\beta}(\cdot, \zeta) \in \mathcal{R}\left(T_{\beta}\right)$,

(ii) $F(\zeta)=\left(F, K_{\beta}(\cdot, \zeta)\right)_{\mathcal{R}\left(T_{\beta}\right)}$ for any $F \in \mathcal{R}\left(T_{\beta}\right)$.

Then, $\mathcal{R}\left(T_{\beta}\right)=H_{\alpha}^{2}\left(\Omega_{n}\right)$ (thought of as an RKH space with the reproducing kernel $K_{\beta}$ ) will be denoted by $H\left(K_{\beta}\right)$. On the other hand, by a result of M.M. Djrbashian and A.H. Karapetyan (cf. Proposition 4.3 in [36], p. 107)

$$
\mathcal{N}\left(T_{\alpha}\right)=L_{\alpha}^{2}\left(\Omega_{n}\right) \ominus H_{\alpha}^{2}\left(\Omega_{n}\right),
$$

hence $H_{\alpha}^{2}\left(\Omega_{n}\right)$ is a closed subspace of $L_{\alpha}^{2}\left(\Omega_{n}\right)$. Next, note that

$$
H\left(K_{\beta}\right)=L^{2} H\left(\Omega_{n}, \gamma_{\alpha}\right)
$$

(the identity is an isometry) if and only if $\mathcal{N}\left(T_{\beta}\right)=\mathcal{N}\left(T_{\alpha}\right)$.

Let $F \in H_{\alpha}^{2}\left(\Omega_{n}\right)$. Then, $\|F\|_{H\left(K_{\beta}\right)} \leq\|f\|_{\gamma_{\alpha}}$ for every $f \in L^{2}\left(\Omega_{n}, \gamma_{\alpha}\right)$ with $T_{\beta} f=F$. Next (by Theorem 2.1 in [36]) $T_{\beta} F=F$. Yet (in view of (46)) in general $F$ is not the element of minimum $\|\cdot\|_{\gamma_{\alpha}}$ norm in the fibre of $T_{\beta}$ over $F$.

By (45), we may define $h_{\beta}: \Omega_{n} \times \Omega_{n} \rightarrow \mathbb{C}$ by setting $h_{\beta}(\zeta, z)=h_{z}(\zeta)$ where $h_{z}$ is given by (44). Then $h_{\beta}(\zeta, z)$ is referred to as the Djrbashian kernel of $\Omega_{n}$.

Let $\mathcal{C}: \mathbb{B}^{n} \rightarrow \Omega_{n}$ be the Cayley transform, that is,

$$
\mathcal{C}(z)=\left(i \frac{1+z_{1}}{1-z_{1}}, \frac{i}{1-z_{1}} z^{\prime}\right), \quad 1-z_{1} \neq 0
$$

and let us set $\Omega_{n, r}=\mathcal{C}\left(\mathbb{B}_{r}^{n}\right)$ where $\mathbb{B}_{r}^{n}=B_{r}(0)$. By a result in [28] (cf. Theorem 1, p. 51) $H_{\alpha}^{2}\left(\Omega_{n}\right)$ is the RKH space $H\left(K_{\beta}\right)$ with the reproducing kernel

$$
\begin{gathered}
K_{\beta}(\zeta, z)=\left|2^{n-1+\beta} c_{n, \beta}\right|^{2} \times \\
\times \int_{\Omega_{n}} \frac{\left[\operatorname{Im}\left(\omega_{1}\right)-\left|\omega^{\prime}\right|^{2}\right]^{2 \operatorname{Re}(\beta)-\alpha} d \mu(\omega)}{\left[i\left(\bar{\omega}_{1}-\zeta_{1}\right)-2\left\langle\zeta^{\prime}, \omega^{\prime}\right\rangle\right]^{n+1+\bar{\beta}}} .
\end{gathered}
$$

Moreover, let $\left\{r_{N}\right\}_{N \geq 1}$ be a sequence of positive numbers such that $r_{N} \uparrow 1$ as $N \rightarrow \infty$ and let us set $D_{N}=\Omega_{n, r_{N}}$. Then (again cf. Theorem 1 in [28], p. 51) for every $F \in H_{\alpha}^{2}\left(\Omega_{n}\right)$ the unique $f^{*} \in L_{\alpha}^{2}\left(\Omega_{n}\right)$ such that $T_{\beta} f^{*}=F$ and $\|F\|_{H\left(K_{\beta}\right)}=\left\|f^{*}\right\|_{\gamma_{\alpha}}$ is given by

$$
f^{*}(\zeta)=\lim _{N \rightarrow \infty} \int_{D_{N}} F(z) h_{\beta}(\zeta, z)\left(\operatorname{Im}\left(z_{1}\right)-\left|z^{\prime}\right|^{2}\right)^{\alpha} d \mu(z)
$$

in the sense of $L_{\alpha}^{2}\left(\Omega_{n}\right)$ convergence. 
We recall that $H_{\alpha}^{2}\left(\Omega_{n}\right)$ is closed in $L_{\alpha}^{2}\left(\Omega_{n}\right)$. Besides, from that

$$
\left|\delta_{z}(F)\right|=\left|\left(T_{\beta} F\right)(z)\right|=\left|\left(F, h_{z}\right)_{\gamma_{\alpha}}\right| \leq\|F\|_{\gamma_{\alpha}}\left\|h_{z}\right\|_{\gamma_{\alpha}}
$$

so that $\delta_{z}: H_{\alpha}^{2}\left(\Omega_{n}\right) \rightarrow \mathbb{C}$ is continuous. Thus $\gamma_{\alpha} \in A W\left(\Omega_{n}\right)$ (as already stated in Example 4$)$ and in view of

$$
\begin{gathered}
\left(T_{\beta} f\right)(z)=\int_{\Omega_{n}} f(\zeta) \overline{h_{\beta}(\zeta, z)} d \mu_{\alpha}(\zeta), \\
d \mu_{\alpha}(\zeta)=\left[\operatorname{Im}\left(\zeta_{1}\right)-\left|\zeta^{\prime}\right|^{2}\right]^{\alpha} d \mu(\zeta),
\end{gathered}
$$

together with Theorem 2.1 in [36], p. 101, the $\gamma_{\alpha}$-Bergman kernel of $\Omega_{n}$ may be identified among the Djrbashian kernels

$$
h_{\beta}(\zeta, z), \operatorname{Re}(\beta)>\frac{\alpha-1}{2},
$$

as the one corresponding to $\beta=\alpha$. Indeed

$$
h_{\alpha}(\zeta, z)=\frac{2^{n-1+\alpha} c_{n, \alpha}}{\left[i\left(\bar{z}_{1}-\zeta_{1}\right)-2\left\langle\zeta^{\prime}, z^{\prime}\right\rangle\right]^{n+1+\alpha}}
$$

is holomorphic in $\zeta$, and hence by the uniqueness statement in the Riesz representation theorem, $h_{\alpha}(\zeta, z)$ is the $\gamma_{\alpha}$-Bergman kernel of $\Omega_{n}$. Moreover, by the reproducing property of $K_{\alpha}(\zeta, z)$ one actually has $K_{\alpha}(\zeta, z)=h_{\alpha}(\zeta, z)$ for every $\alpha>-1$.

\subsection{Djrbashian Kernels and Quantum States}

Let us think of $\Omega_{n}$ as the classical phase space of some mechanical system, and let $E=\Omega_{n} \times \mathbb{C}$ be the trivial complex line bundle over $\Omega_{n}$, with the Hermitian metric $H_{\alpha}\left(s^{0}, s^{0}\right)=\rho^{\alpha}$ where $\rho(\zeta)=$ $\operatorname{Im}\left(\zeta_{1}\right)-\left|\zeta^{\prime}\right|^{2}$ and the holomorphic frame $s^{0}: \Omega_{n} \rightarrow E$ is $s^{0}(\zeta)=(\zeta, 1)$. By a result in $[28] H_{\alpha}^{2}\left(\Omega_{n}\right)$ is the space of quantum states of $\Omega_{n}$ and, by a result in [9], there is an anti-holomorphic embedding $\mathcal{K}_{\alpha}: \Omega_{n} \rightarrow \mathbb{C P}\left(H_{\alpha}^{2}\left(\Omega_{n}\right)\right)$. Moreover, if the pair $(n, \alpha)$ satisfies one of the following conditions:

(i) $\quad n=\mathcal{M}_{4}$ and $\alpha \in(-1,0) \cup(0,+\infty)$,

(ii) $n=\mathcal{M}_{4}+1$ and $\alpha \in(0,+\infty)$,

(iii) $n=\mathcal{M}_{4}+3$ and $\alpha \in(-1,0)$,

(where $\mathcal{M}_{4}=4 k$ for some $k \in \mathbb{N}$ ) then the transition probability amplitude $a_{\alpha}(\zeta, z)=\left\langle\mathcal{K}_{\alpha}(\zeta), \mathcal{K}_{\alpha}(z)\right\rangle$ satisfies the rule

$$
\int_{\Omega_{n}} a_{\alpha}(\zeta, w) a_{\alpha}(z, \zeta) c d \mu_{L}(\zeta)=a_{\alpha}(z, w)
$$

for some constant $c>0$ depending only on $n$ and $\alpha$. Cf. Theorem 3 in [28], p. 57. The proof consists essentially of the following three steps.

Step 1. If $K_{\alpha}(\zeta, z)$ is the $\gamma_{\alpha}$-Bergman kernel of $\Omega_{n}$ then the map

$$
\mathcal{K}_{\alpha}: \Omega_{n} \rightarrow \mathbb{C P}\left(H_{\alpha}^{2}\left(\Omega_{n}\right)\right), \quad \mathcal{K}_{\alpha}(z)=\left[K_{\alpha}(\cdot, z)\right], \quad z \in \Omega_{n},
$$

is an anti-holomorphic embedding.

Step 2. The identity

$$
a_{\alpha}(z, w)=\int_{\Omega_{n}} a_{\alpha}(\zeta, w) \overline{a_{\alpha}(\zeta, z)} K_{\alpha}(\zeta, \zeta) \gamma_{\alpha}(\zeta) d \mu(\zeta)
$$

holds for any $z, w \in \Omega_{n}$. 
Step 3. If $(n, \alpha)$ satisfies one of the assumptions (i)-(iii) above, then there is a constant $C>0$ depending only on $n$ and $\alpha$ such that the weight $\gamma_{\alpha}(\zeta)=\left[\operatorname{Im}\left(\zeta_{1}\right)-\left|\zeta^{\prime}\right|^{2}\right]^{\alpha}$ satisfies the complex Monge-Ampère equation

$$
\operatorname{det}\left[\frac{\partial^{2} \log \gamma(\zeta)}{\partial \zeta_{j} \partial \bar{\zeta}_{k}}\right]=(-1)^{n(n+1) / 2} C \frac{1}{n !} \gamma(\zeta) K_{\gamma}(\zeta, \zeta)
$$

Cf. also E. Barletta and S. Dragomir [107], V. Faber and J. Mycielski [108], J. Mycielski and Świerczkowski [109],

\section{Forelli-Rudin-Ligocka-Peloso Asymptotic Expansion Formula}

Let $\Omega=\left\{z \in \mathbb{C}^{n}: \varphi(z)<0\right\}$ be a smoothly bounded strictly pseudoconvex domain where $\varphi$ is such that the Levi form $L_{\varphi}$ satisfies

$$
L_{\varphi}(w) \xi \geq C_{1}|\xi|^{2}, \quad \xi \in \mathbb{C}^{n}
$$

for $\varphi(w)<\delta_{0}, \delta_{0}>0$, and $C_{1}$ depending only on $\Omega$. Let us set

$$
\begin{gathered}
\Psi(\zeta, z)=[F(\zeta, z)-\varphi(z)] \chi(|\zeta-z|)+(1-\chi(|\zeta-z|))|\zeta-z|^{2}, \\
F(\zeta, z)=-\sum_{j=1}^{n} \frac{\partial \varphi}{\partial z_{j}}(z)\left(\zeta_{j}-z_{j}\right)-\frac{1}{2} \sum_{j, k=1}^{n} \frac{\partial^{2} \varphi}{\partial z_{j} \partial z_{k}}(z)\left(\zeta_{j}-z_{j}\right)\left(\zeta_{k}-z_{k}\right),
\end{gathered}
$$

where $\chi$ is a $C^{\infty}$ cut-off function of the real variable $t$, with $\chi(t)=1$ for $|t|<\epsilon_{0} / 2$ and $\chi(t)=0$ for $|t| \geq 3 \epsilon_{0} / 4$. We recall (cf. Theorem 1 in [21], p. 287)

Theorem 3. For any nonnegative integer $m \in\{0,1,2, \cdots\}$ the weight $|\varphi|^{m}$ is admissible, that is, $|\varphi|^{m} \in$ $A W(\Omega)$. Let $K_{m}(\zeta, z)$ be the $|\varphi|^{m}$-Bergman kernel for $L^{2} H\left(\Omega,|\varphi|^{m}\right)$. Then

$$
K_{m}(\zeta, z)=C_{\Omega}|\nabla \varphi(z)|^{2} \cdot \operatorname{det} L_{\varphi}(z) \cdot \Psi(\zeta, z)^{-(n+1+m)}+E(\zeta, z)
$$

where $E \in C^{\infty}(\bar{\Omega} \times \bar{\Omega} \backslash \Delta), \Delta$ is the diagonal in $\partial \Omega \times \partial \Omega$, and $E$ satisfies the estimate

$$
|E(\zeta, z)| \leq C_{\Omega}^{\prime}|\Psi(\zeta, z)|^{-(n+1+m)+1 / 2}|\log | \Psi(\zeta, z)|| .
$$

Theorem 3 extends C. Fefferman's asymptotic expansion formula for the Bergman kernel of a strictly pseudoconvex domain (cf. [16] for $m=0$ ) to the case of $|\varphi|^{m}$-Bergman kernels, $m \in\{1,2, \cdots\}$. The result is due to M.M. Peloso (cf. Lemma 2.2 in [17], p. 229). However, M.M. Peloso claims Theorem 3 is implicit in [19], while E. Ligocka employs an older idea by F. Forelli and W. Rudin (cf. [20]). Aside from the correct credit, which certainly goes to M.M. Peloso, the history of Theorem 3 demonstrates the attention shown by the mathematical community devoted to complex analysis to an argument born with the celebrated work by C. Fefferman (cf. op. cit.), and emphasizes the recognition of the relevance of that argument.

Part of the proof of Theorem 3, relating $K_{m}(\zeta, z)$ to the ordinary Bergman kernel of the "suspended" domain

$$
\left\{(z, \xi) \in \Omega \times \mathbb{C}^{m}: \varphi(z)+|\xi|^{2}<0\right\},
$$


does work for an arbitrary admissible weight $\gamma \in A W(\Omega)$. Precisely, if $K_{\Omega_{m}}((z, \xi),(w, \eta))$ is the Bergman kernel of the domain

$$
\Omega_{m}=\left\{(z, \xi) \in \Omega \times \mathbb{C}^{m}:|\xi|^{2 m}<\gamma(z)\right\}
$$

then

$$
K_{\gamma}(z, w)=\frac{\omega_{2 m-1}}{2 m} K_{\Omega_{m}}((z, 0),(w, 0)),
$$

where $\omega_{N}$ is the "area" of the sphere $S^{N} \subset \mathbb{R}^{N+1}$ (this is of course Formula (14) in Section 2 of the present survey). When $\gamma=|\varphi|^{m}, m \in\{1,2,3, \cdots\}$, the domain $\Omega_{m}$ is strictly pseudoconvex and (50) follows from (52) together with Fefferman's asymptotic expansion formula for $K_{\Omega_{m}}$

$$
\begin{gathered}
K_{\Omega_{m}}((z, \xi),(w, \eta))=\text { const. }\left|\nabla \varphi_{1}(z, \xi)\right|^{2} \cdot \operatorname{det} L_{\varphi_{1}}(z, \xi) \cdot \\
\cdot \Psi((z, \xi),(w, \eta))^{-(n+1+m)}+E((z, \xi),(w, \eta)), \\
|E((z, \xi),(w, \eta))| \leq \\
\leq \text { const. } \mid \Psi\left((z, \xi),\left.(w, \eta)\right|^{-(n+1+m)+1 / 2}|\log | \Psi((z, \xi),(w, \eta))||,\right. \\
E \in C^{\infty}\left(\bar{\Omega}_{m} \times \bar{\Omega}_{m} \backslash \Delta_{1}\right),
\end{gathered}
$$

where $\Psi((z, \xi),(w, \eta))$ is defined as in (49) (modulo obvious modifications) and $\varphi_{1}(z, \xi)=\varphi(z)+|\xi|^{2}$, and $\Delta_{1}$ is the diagonal in $\partial \Omega_{m} \times \partial \Omega_{m}$.

It should be observed that the Fefferman-like asymptotic expansion of a weighted Bergman kernel is known (cf. Theorem 3 above) only for the points of the curve

$$
C:(-1,+\infty) \rightarrow W(\Omega), \quad C(\alpha)=|\varphi|^{\alpha} \in A W(\Omega), \quad \alpha>-1,
$$

corresponding to the integer values of the parameter. Extending Theorem 3 to all weights $\gamma \in A W(\Omega)$ is still an open problem. By a result in [21] (cf. Theorem 2, p. 289) the curve (53) is discontinuous, and every point of $C$ is an isolated point in $W(\Omega)$. The result may be looked at as a measure of the amount of job (deriving an asymptotic expansion formula for $K_{\gamma}(\zeta, z)$ ) left unsolved. As a step in this direction, by a result in [21] (cf. Theorem 4, p. 293) for every $h \in B_{1 / 2}(0) \subset L^{\infty}(\Omega)$ there is $E_{h} \in C^{\infty}(\Omega \times \Omega)$ such that

$$
\begin{gathered}
K_{(1+h)|\varphi|^{m}}(z, w)= \\
=C_{\Omega}|\nabla \varphi(w)|^{2} \cdot \operatorname{det} L_{\varphi}(w) \cdot \Psi(z, w)^{-(n+1+m)}+E_{h}(z, w), \\
\left|E_{h}(z, w)\right| \leq C\left\{|\Psi(z, w)|^{-(n+1+m)+1 / 2}|\log | \Psi(z, w)||+\right. \\
\left.+|\varphi(z)|^{-(n+1+m) / 2}|\varphi(w)|^{-(n+1+m) / 2}[1+F(z)+F(w)+F(z) F(w)]\right\}
\end{gathered}
$$

where $F(z)=|\varphi(z)|^{3 / 2}+|\varphi(z)|^{1 / 2}|\log | \varphi(z)||$ and $C$ is a constant depending only on $\Omega$ and on $m \geq 1$, $m>n-1$.

The proof of (54) and (55) relies on (50) and (51) and on the analyticity of the weighted Bergman kernel as a function of weight, as described by us in Section 2 of this work (and of course due to [2]).

Remarkable progress (towards obtaining analogs to Fefferman's asymptotic expansion for weighted Bergman kernels $K_{\gamma}(z, \zeta)$ for more general classes of weights $\gamma \in A W(\Omega)$ ) was obtained by M. Englis, [22], for a class of weights behaving like a power of the defining function. To state the result 
in [22] let $\Omega=\{\varphi<0\} \subset \mathbb{C}^{n}$ be a smoothly bounded strictly peudoconvex domain and let us recall that a function $F \in C^{\infty}(\bar{\Omega} \times \bar{\Omega})$ is said to be almost-sesquianalytic if

$$
\frac{\partial F}{\partial \bar{z}_{j}}(z, \zeta), \quad \frac{\partial F}{\partial \zeta_{j}}(z, \zeta), \quad 1 \leq j \leq n,
$$

vanish to infinite order on the diagonal $z=\zeta$. Additionally, given a function $f: \Omega \rightarrow \mathbb{R}$, an almost-sesquianalytic function $F \in C^{\infty}(\bar{\Omega} \times \bar{\Omega})$ is an (almost-sesquianalytic) extension of $f$ if $F(z, z)=f(z)$ for any $z \in \bar{\Omega}$ and $\overline{F(z, \zeta)}=F(\zeta, z)$. As a consequence of strict pseudoconvexity $-\varphi$ admits an almost-sesquilinear extension $\Phi(z, \zeta)$ such that

$$
2 \operatorname{Re} \Phi(z, \zeta) \geq|\varphi(z)|+|\varphi(\zeta)|+C|z-\zeta|^{2}
$$

for some $C>0$ and some $\epsilon>0$ and any $z, \zeta \in \bar{\Omega}$ with $|z-\zeta|<\epsilon$. Let us fix such an almost-sesquilinear extension $\Phi(z, \zeta)$ of $|\varphi|$. The main result in [22] is that any weight $\gamma \in W(\Omega)$ of the form

$$
\gamma(z)=|\varphi(z)|^{\alpha} \exp [g(z)], \quad \alpha>-1, g \in C^{\infty}(\bar{\Omega}), \quad z \in \Omega,
$$

is admissible and the corresponding $\gamma$-Bergman kernel admits the following asymptotic expansion

$$
\begin{gathered}
K_{|\varphi|^{\alpha} e^{g}}(z, \zeta)= \\
= \begin{cases}a(z, \zeta) \Phi(z, \zeta)^{-(n+\alpha+1)}+b(z, \zeta) \log \Phi(z, \zeta) & \alpha \in \mathbb{Z}_{+}, \\
a(z, \zeta) \Phi(z, \zeta)^{-(n+\alpha+1)}+b(z, \zeta) & \alpha \in(-1,+\infty) \backslash \mathbb{Z},\end{cases}
\end{gathered}
$$

for some almost-sesquianalytic functions $a, b \in C^{\infty}(\bar{\Omega} \times \bar{\Omega})$ and any $z, \zeta \in \Omega$. Moreover,

$$
a(z, z)=\frac{\Gamma(n+\alpha+1)}{\Gamma(\alpha+1)} J(-\varphi)(z) e^{-g(z)}
$$

for every $z \in \partial \Omega$. Here $J$ is the complex Monge-Ampére operator, that is,

$$
J(u)=(-1)^{n} \operatorname{det}\left(\begin{array}{cc}
u & u_{z_{j}} \\
u_{\bar{z}_{k}} & u_{z_{j}} \bar{z}_{k}
\end{array}\right) .
$$

The leading term (56) was first computed by L. Hörmander (cf. [14]) for $\alpha=0$ and by E. Ligocka (cf. [19]) for $g=0$ and $\alpha>-1$. The fact that $K_{|\varphi|^{\alpha} e^{g}} \in C^{\infty}(\bar{\Omega} \times \bar{\Omega} \backslash \Delta)$ for $\alpha \neq 0$ was proved by M.M. Peloso (cf. [18]) and the same result for $\alpha=0$ follows by a straightforward (according to [22], p. 1422) modification of N. Kerzman's arguments (cf. [13]) for the unweighted Bergman kernel.

By a result of M. Englis (cf. [110]) there is a set $U \subset \mathbb{C}$ without accumulation points such that for any $z, \zeta \in \Omega$ the function $\alpha \in(-1,+\infty) \longmapsto K_{|\varphi| \alpha}(z, \zeta) \in \mathbb{C}$ extends to a holomorphic function on $\mathbb{C} \backslash U$ having at most poles at the points of $U$. The prototypical situation appears to be that of the unit ball $\Omega=\mathbb{B}^{n}$ with $\varphi(z)=|z|^{2}-1$ where the $|\varphi|^{\alpha}$-Bergman kernel is

$$
K_{|\varphi|^{\alpha}}(z, \zeta)=\frac{(\alpha+1) \cdots(\alpha+n)}{\pi^{n}}(1-z \cdot \bar{\zeta})^{-\alpha-n-1},
$$

hence $U=\varnothing$, and the extension of $\alpha \mapsto K_{\mid \varphi^{\alpha}}(z, \zeta)$ has zeros (rather than poles) at $\alpha \in$ $\{-1,-2, \cdots,-n\}$. We expect that $\alpha \in(-1,+\infty) \mapsto K_{|\varphi|^{\alpha}} \in H A(\Omega)$ extends to a holomorphic function $\mathbb{C} \backslash U \rightarrow H A(\Omega)$ (as a function of one complex variable with values in a complex Fréchet space, cf. Definition 3.30 in [111], p. 82).

See also M. Englis (cf. [112]) for weighted Bergman kernels with logarithmic weights. 
C. Fefferman discovered the asymptotic expansion (50) (for $m=0$ ) in [16] and used it as a tool to prove his celebrated result that biholomorphisms of smoothly bounded strictly pseudoconvex domains extend smoothly to the boundary. Successively, (50) proved useful in C. Fefferman's first approach (cf. [15]) to the construction of the Fefferman metric $F$ on $\partial \Omega \times S^{1}$ (briefly discussed by us in Section 3.2 of the present survey). Although [15] proposes two other methods of calculation of $F$ (replacing $K(z, z)^{-1 /(n+1)}$ in the potential $U(\zeta, z)$ first by a solution $u(z)$ to the Dirichlet problem

$$
\begin{gathered}
J(u)=(-1)^{n} \operatorname{det}\left(\begin{array}{cc}
u & u_{z_{j}} \\
u_{\bar{z}_{k}} & u_{z_{j} \bar{z}_{k}}
\end{array}\right)=1 \text { in } \Omega, \\
u=0 \quad \text { on } \partial \Omega
\end{gathered}
$$

for the complex Monge-Ampère equation, and then by the 2-jet of a solution to the same equation) the question remains as to whether

$$
|\zeta|^{2 /(n+1)} K_{\gamma}(z, z)^{-1 /(n+1)}, \quad z \in \Omega, \quad \zeta \in \mathbb{C} \backslash\{0\},
$$

can be used for constructing new Lorentzian metrics $F_{\gamma}$ on $\partial \Omega \times S^{1}$. As (50) is already available, $F_{|\varphi|^{m}}$ may be derived by following the kernel-based approach in [15]. The problem of building weighted analogs to Fefferman metrics for arbitrary weights $\gamma \in A W(\Omega)$ is open.

\section{Computability of $\gamma$-Bergman Kernels}

Ordinary Bergman kernels of domains in $\mathbb{C}^{n}$ were explicitly computed only for a handful of examples, such as the unit ball (cf. [113]), or complex ovals (cf. J.P. D'Angelo [114], G. Francsics and $\mathrm{N}$. Hanges [115]). This is mainly due to the difficulty of producing explicit complete orthonormal systems in $L^{2} H(\Omega)$, for the various domains $\Omega \subset \mathbb{C}^{n}$ at hand, and the problem of course remains for the weighted case. For instance, let $\Omega \subset \mathbb{C}^{n}$ be a (not necessarily bounded) Reinhardt domain containing the origin, and let $\mathcal{J}=\left\{\alpha \in \mathbb{Z}_{+}^{n}: \phi_{\alpha} \in L^{2}(\Omega)\right\}$, where $\phi_{\alpha}(z)=z^{\alpha}$. Then $\left\{\phi_{\alpha}: \alpha \in \mathcal{J}\right\}$ is a complete orthogonal system in $L^{2} H(\Omega)$ and the Bergman kernel of $\Omega$ may be written as

$$
K_{\Omega}(\zeta, z)=\sum_{\alpha \in \mathcal{J}} \frac{(\zeta \bar{z})^{\alpha}}{\left\|\phi_{\alpha}\right\|_{L^{2}(\Omega)}^{2}} .
$$

Note that so far one only exhibited an orthogonal system, and that the calculation of the norms $\left\|\phi_{\alpha}\right\|_{L^{2}(\Omega)}$ requires rather involved calculations. In particular cases, such as

$$
\Omega_{p}=\left\{(z, w) \in \mathbb{C}^{n} \times \mathbb{C}^{m}:\|z\|^{2}+\|w\|^{2 p}<1\right\}, \quad p>0,
$$

$K_{\Omega_{p}}$ may be expressed in terms of elementary functions, cf. J. D'Angelo [114]. Additionally, if $\Omega$ is a complex oval

$$
\begin{gathered}
\Omega_{\mathbf{a}}=\left\{z \in \mathbb{C}^{n}: \sum_{j=1}^{n}\left|z_{j}\right|^{2 a_{j}}<1\right\}, \\
\mathbf{a}=\left(a_{1}, \cdots, a_{n}\right), \quad a_{j} \in \mathbb{N}, \quad 1 \leq j \leq n,
\end{gathered}
$$

$K_{\Omega_{\mathrm{a}}}$ may be expressed in terms of generalized hypergeometric functions, cf. G. Francsics and N. Hanges [115]. H.P. Boas, S. Fu, and E.J. Straube considered (cf. [49]) $\omega=\left\{(z, \zeta) \in \mathbb{C} \times \mathbb{C}^{n}:|z|<\right.$ $\gamma(\zeta)\}$, where $\gamma \in L^{\infty}(\Omega)$ is a continuous and positive function and $\Omega \subset \mathbb{C}^{n}$ is a bounded domain. Then, they computed the Bergman kernel $K_{\Omega_{m}}$ of the domain

$$
\Omega_{m}=\left\{(z, \zeta) \in \mathbb{C}^{m} \times \mathbb{C}^{n}:\|z\|<\gamma(\zeta)\right\}
$$


essentially by differentiating the Bergman kernel $K_{\omega}$. Domains such as

$$
\left\{\left(z_{1}, z_{2}, z_{3}\right):\left(\left|z_{1}\right|^{2 p}+\left|z_{2}\right|^{4}\right)^{1 / \lambda}+\left|z_{3}\right|^{2 / q}<1\right\}
$$

and

$$
\left\{(z, \zeta) \in \mathbb{C}^{n} \times \mathbb{C}^{m}:\|z\|<\exp \left(-a\|\zeta\|^{2}\right)\right\}
$$

(the Fock-Bargmann-Hartogs domain) were respectively dealt with by T. Beberok [116] and A. Yamamori [87].

A new method of calculating (unweighted) Bergman kernels for certain families of Hartogs domains was recently devised by Z. Huo [50], allowing him to rediscover some of the known examples and obtain new explicit formulas. To illustrate the ideas in [50], which appear to us of a certain value, let us look at the following example. Let

$$
\Omega=\left\{(z, \zeta) \in \mathbb{C}^{2}:|z|^{2 a}+|\zeta|^{2}<1\right\}, \quad a>0 .
$$

Next, let us think of $\zeta$ as a parameter $\zeta \in \mathbb{B}^{1} \subset \mathbb{C}$, so that to associate with (57) the following family of plane domains

$$
\left\{\Omega_{\zeta}\right\}_{|\zeta|<1}, \quad \Omega_{\zeta}=\left\{z \in \mathbb{C}: \frac{|z|^{2}}{\left(1-|\zeta|^{2}\right)^{1 / a}}<1\right\} .
$$

For each $\eta \in \mathbb{C}$ with $|\eta|<1$

$$
\phi: \Omega_{\eta} \rightarrow \mathbb{B}^{1}, \quad \phi(z)=\frac{z}{\left(1-|\eta|^{2}\right)^{1 /(2 a)}}, \quad z \in \Omega_{\eta},
$$

is a biholomprphism of $\Omega_{\eta}$ onto the unit disc $\mathbb{B}^{1}$. The Bergman kernels of $\Omega_{\eta}$ and $\mathbb{B}^{1}$ are then related by

$$
\begin{gathered}
K_{\Omega_{\eta}}(z, \zeta)=\frac{\partial \phi}{\partial z}(z) \overline{\frac{\partial \phi}{\partial z}(\zeta)} K_{\mathbb{B}^{1}}(\phi(z), \phi(\zeta)), \quad z, \zeta \in \Omega_{\eta} \\
K_{\mathbb{B}^{1}}(z, \zeta)=\frac{1}{\pi} \frac{1}{(1-z \bar{\zeta})^{2}}, \quad z, \zeta \in \mathbb{B}^{1}
\end{gathered}
$$

hence

$$
K_{\Omega_{\eta}}(z, \zeta)=\frac{\left(1-|\eta|^{2}\right)^{1 / a}}{\pi\left[\left(1-|\eta|^{2}\right)^{1 / a}-z \bar{\zeta}\right]^{2}} .
$$

Moreover, let us consider the differential operator

$$
D=\frac{1}{\pi}\left(\frac{1-|\eta|^{2}}{(1-w \bar{\eta})^{2}}\right)^{1 / a}\left\{\left(1+\frac{1}{a}\right) I+\frac{z}{a} \frac{\partial}{\partial z}\right\}
$$

where $I$ is the identity operator. Finally, applying $D$ to (58) produces the Bergman kernel $K_{\Omega}(z, w, \zeta, \eta)$. Cf. Z. Huo [50], pp. 2-3. The choice of differential operator $D$ is rather heuristic (the same remark applies to the choice of differential operators $D_{U^{\alpha}}$ and $D_{V^{\gamma}}$ in [50], p. 9) and it is still unclear whether Huo's method carries over to balls with both radius and center depending on the parameter. For instance, let us consider the worm domain

$$
\Omega=\left\{(z, w) \in \mathbb{C}^{2}:\left|z-e^{i \log |w|^{2}}\right|^{2}-1+\varphi\left(\log |w|^{2}\right)<0\right\}
$$


where $\varphi \in C^{\infty}(\mathbb{R})$ is an even and convex function such that (i) $\varphi(t) \geq 0, \varphi^{-1}(0)=[-\mu, \mu], \mu>0$, (ii) there is $a>0$ such that $|t|>a \Longrightarrow \varphi(t)>1$, and (iii) $\varphi(t)=1 \Longrightarrow \varphi^{\prime}(t) \neq 0$. If $\omega=\{w \in \mathbb{C}$ : $\left.1-\varphi\left(\log |w|^{2}\right)>0\right\}$ then $(z, w) \in \Omega \Longrightarrow w \in \omega$. Hence one may associate to $\Omega$ the family of domains

$$
\Omega_{w}=B_{r(w)}\left(e^{i \log |w|^{2}}\right), \quad r(w)=\left[1-\varphi\left(\log |w|^{2}\right)\right]^{1 / 2}, \quad w \in \omega .
$$

Then

$$
\phi: \Omega_{w} \rightarrow \mathbb{B}^{1}, \quad \phi(z)=\frac{z-e^{i \log |w|^{2}}}{\left[1-\varphi\left(\log |w|^{2}\right)\right]^{1 / 2}},
$$

is a biholomorphism so that, by once again taking into account the transformation law of Bergman kernels under biholomorphisms, the Bergman kernel of $\mathcal{W}_{w}$ is

$$
K_{\mathcal{W}_{w}}(z, \zeta)=\frac{1}{\pi} \frac{1-\varphi\left(\log |w|^{2}\right)}{\left[1-\varphi\left(\log |w|^{2}\right)-\left(z-e^{i \log |w|^{2}}\right)\left(\bar{\zeta}-e^{-i \log |w|^{2}}\right)\right]^{2}}
$$

for any $w \in \omega$. It is an open problem whether a differential operator $D$ may be produced such that $D K_{\mathcal{W}_{w}}$ gives the Bergman kernel of $\mathcal{W}$.

\section{Cartan Connections and Fefferman's Theorem}

Let $G \rightarrow P \rightarrow M$ be a principal bundle, with the structure Lie group $G$. Let $\tilde{G}$ be another Lie group, and let us assume that $G$ is a Lie subgroup of $\tilde{G}$ (i.e., $G \subset \tilde{G}$ ). Let $\mathfrak{g}$ and $\tilde{\mathfrak{g}}$ be the Lie algebras of $G$ and $\tilde{G}$, respectively. Let $\omega \in C^{\infty}\left(T^{*}(P) \otimes \tilde{\mathfrak{g}}\right)$ be a $\tilde{\mathfrak{g}}$-valued differential 1-form on $P$. The pair $(P, \omega)$ is a Cartan connection of type $(G, \tilde{G})$ if

(i) For every $p \in P$ the map $\omega_{p}: T_{p}(P) \rightarrow \tilde{\mathfrak{g}}$ is an isomorphism;

(ii) $R_{a}^{*} \omega=\operatorname{ad}\left(a^{-1}\right) \omega$ for any $a \in G$;

(iii) $\omega\left(A^{*}\right)=A$ for any left invariant vector field $A \in \mathfrak{g}$, where $A^{*} \in \mathfrak{X}(P)$ is the fundamental vector field associated to $A$.

If $(P, \omega)$ and $\left(P^{\prime}, \omega^{\prime}\right)$ are Cartan connections of the same type, then a diffeomorphism $\varphi: P \rightarrow P^{\prime}$ is an isomorphism of $(P, \omega)$ onto $\left(P^{\prime}, \omega^{\prime}\right)$ if $\varphi^{*} \omega^{\prime}=\omega$.

Let $(P, \omega)$ and $\left(P^{\prime}, \omega^{\prime}\right)$ be Cartan connections of type $(G, \tilde{G})$ with $G$ connected. Then, every isomorphism $\varphi:(P, \omega) \rightarrow\left(P^{\prime}, \omega^{\prime}\right)$ is G-equivariant.

Let $(P, \omega)$ be a Cartan connection of type $(G, \tilde{G})$ and let $(\cdot, \cdot)$ be a positive definite inner product on $\tilde{\mathfrak{g}}$. Let $g$ be the Riemannian metric on $P$ defined by

$$
g_{p}(X, Y)=\left(\omega_{p}(X), \omega_{p}(Y)\right), \quad X, Y \in T_{p}(P) .
$$

Then, $g$ is $G$-admissible; that is, for every $a \in G$ the right translation $R_{a}: P \rightarrow P$ is uniformly continuous with respect to the distance function $d_{g}: P \times P \rightarrow[0,+\infty)$ associated to $g$. In particular, every right translation maps Cauchy sequences to Cauchy sequences, and hence the action of $G$ on $P$ extends to an action of $G$ on the Cauchy completion $\hat{P}$ of $\left(P, d_{g}\right)$, as a topological transformation group.

The Riemannian metric (59) is commonly referred to as the canonical metric of $(P, \omega)$. Of course, the definition of (59) depends on the choice of scalar product on (the choice of linear basis in) $\tilde{\mathfrak{g}}$. Every $C^{1}$ isomorphism of Cartan connections is an isometry with respect to their canonical metrics. We recall the following (cf. Theorem 1.2 in [23], p. 119):

Theorem 4. Let $(P, \omega)$ and $\left(P^{\prime}, \omega^{\prime}\right)$ be two Cartan connections of type $(G, \tilde{G})$. Let $C \subset P$ and $C^{\prime} \subset P^{\prime}$ be two G-invariant closed submanifolds of codimension $\geq 2$. Let us assume that the base manifolds $M=P / G$ and $M^{\prime}=P^{\prime} / G$ are compact and the Lie structure group $G$ is connected. Then every $C^{1}$ isomorphism $\left(P \backslash C, \mathbf{j}^{*} \omega\right) \approx\left(P^{\prime}, \mathbf{j}^{\prime *} \omega^{\prime}\right)$ extends to an isomorphism $(P, \omega) \approx\left(P^{\prime}, \omega^{\prime}\right)$, where $\mathbf{j}: P \backslash C \hookrightarrow P$ and $\mathbf{j}^{\prime}: P^{\prime} \backslash C^{\prime} \hookrightarrow P^{\prime}$ are inclusions. 
Proof. Let $g$ and $g^{\prime}$ be the canonical metrics of $(P, \omega)$ and $\left(P^{\prime}, \omega^{\prime}\right)$. Then, $\mathbf{j}^{*} g$ and $\mathbf{j}^{\prime *} g^{\prime}$ are the canonical metrics of $\left(P \backslash C, \mathbf{j}^{*} \omega\right)$ and $\left(P^{\prime} \backslash C^{\prime}, \mathbf{j}^{\prime *} \omega^{\prime}\right)$. Let $\varphi: P \backslash C \rightarrow P^{\prime} \backslash C^{\prime}$ be an isomorphism of $\left(P \backslash C, \mathbf{j}^{*} \omega\right)$ onto $\left(P^{\prime} \backslash C^{\prime}, \mathbf{j}^{\prime *} \omega^{\prime}\right)$. Then, $\varphi: P \backslash C \rightarrow P^{\prime} \backslash C^{\prime}$ is G-equivariant and $\varphi^{*}\left(\mathbf{j}^{\prime *} g^{\prime}\right)=\mathbf{j}^{*} g$. At this point we may end the proof of Theorem 4 by applying Lemma 2.

Lemma 2. Let $P$ and $P^{\prime}$ be two principal $G$-bundles with $G$-admissible Riemannian metrics $g$ and $g^{\prime}$. Let $C \subset$ $P$ and $C^{\prime} \subset P^{\prime}$ be two G-invariant closed submanifolds of codimension $\geq 2$. Let us assume that $M=P / G$ and $M^{\prime}=P^{\prime} / G$ are compact. Then, any G-equivariant isometry $\varphi: P \backslash C \rightarrow P^{\prime} \backslash C^{\prime}$ of $\left(P \backslash C, \mathbf{j}^{*} g\right)$ onto $\left(P^{\prime} \backslash C^{\prime}, \mathbf{j}^{\prime *} g^{\prime}\right)$ extends to an isometry $\tilde{\varphi}: P \rightarrow P^{\prime}$ of $(P, g)$ onto $\left(P^{\prime}, g^{\prime}\right)$.

Proof. We may assume w.l.o.g. that $P$ and $P^{\prime}$ are connected. There are a priori two natural distance functions on $P \backslash C$, that is, the distance function induced by $d_{g}: P \times P \rightarrow[0,+\infty)$ and the distance function $d_{\mathbf{j}^{*} g}:(P \backslash C) \times(P \backslash C) \rightarrow[0,+\infty)$ associated to the Riemannian metric $\mathbf{j}^{*} g$. As $\operatorname{codim}(C) \geq 2$ any curve in $P$ having end points fixed in $P \backslash C$ can be smoothly approximated by curves in $P \backslash C$. Consequently, the two distance functions coincide

$$
\left.d_{g}\right|_{(P \backslash C) \times(P \backslash C)}=d_{\mathbf{j}^{*} g} \cdot
$$

Then (by (60)), the Cauchy completions of $\left(P \backslash C, d_{\mathbf{j}^{*} g}\right)$ and $\left(P, d_{g}\right)$ coincide, that is, $\widehat{P \backslash C}=\hat{P}$. By a well-known result in differential geometry (cf. e.g., Theorem 11.1 in [117], p. 60-61), a mapping of Riemannian manifolds is an isometry (a metric preserving diffeomorphism) if and only if it is distance preserving. For the moment we use only the trivial part of this statement. That is, since the map $\varphi: P \backslash C \rightarrow P^{\prime} \backslash C^{\prime}$ is an isometry (i.e., $\varphi: P \backslash C \rightarrow P^{\prime} \backslash C^{\prime}$ is a diffeomorphism and $\left.\varphi^{*}\left(\mathbf{j}^{\prime *} g^{\prime}\right)=\mathbf{j}^{*} g\right)$, it must be distance preserving (i.e., $d_{\mathbf{j}^{\prime *} g^{\prime}}(\varphi(p), \varphi(q))=d_{\mathbf{j}^{*} g}(p, q)$ for any $\left.p, q \in P \backslash C\right)$. Consequently, $\varphi: P \backslash C \rightarrow P^{\prime} \backslash C^{\prime}$ extends to a distance-preserving map $\hat{\varphi}: \widehat{P \backslash C} \rightarrow \widehat{P^{\prime} \backslash C^{\prime}}$, and therefore to a map $\hat{\varphi}: \hat{P} \rightarrow \widehat{P^{\prime}}$. Here the distance functions referred to are $\widehat{d_{g}}: \hat{P} \times \hat{P} \rightarrow[0,+\infty)$ and $\widehat{d_{g^{\prime}}}: \widehat{P^{\prime}} \times \widehat{P^{\prime}} \rightarrow$ $[0,+\infty)$, induced by $d_{g}$ and $d_{g^{\prime}}$. There is a natural injection of $P$ in $\hat{P}$, as an open subset, such that $\left.\widehat{d_{g}}\right|_{P \times P}=d_{g}$. As $\hat{\varphi}: \hat{P} \rightarrow \widehat{P}^{\prime}$ is a homeomorphism, the sets

$$
U=\hat{\varphi}^{-1}\left[\hat{\varphi}(P) \cap P^{\prime}\right], \quad U^{\prime}=\varphi(P) \cap P^{\prime},
$$

are open in $P$ and $P^{\prime}$, respectively. Then, $\hat{\varphi}: U \rightarrow U^{\prime}$ is a distance-preserving homeomorphism (the distances alluded to are the restrictions of $d_{g}$ and $d_{g^{\prime}}$ to $U$ and $U^{\prime}$ ). Now we may apply the hard part in Theorem 11.1 of [117], p. 61, to conclude that $\hat{\varphi}: U \rightarrow U^{\prime}$ is an isometry (with respect to the pullbacks of $g$ and $g^{\prime}$ to $U$ and $U^{\prime}$ ) and in particular a diffeomorphism. Thus, given $k \geq 1$, there is a unique $C^{k}$ manifold structure on $\Omega=\hat{\varphi}(P) \cap P^{\prime}$ such that $\hat{\varphi}: P \rightarrow \Omega$ and $P^{\prime} \hookrightarrow \Omega$ are $C^{k}$ maps. As $\hat{\varphi}$ is $G$-equivariant, $\Omega$ is $G$-invariant and (the total space of) a principal G-bundle. Clearly $\Omega$ is connected, hence $\Omega / G$ is a connected manifold such that $P^{\prime} / G$ is a both open and compact subset of $\Omega / G$. Thus $P^{\prime} / G=\Omega / G$, implying that $P^{\prime}=\Omega$. It follows that $\hat{\varphi}(P) \subset P^{\prime}$. Interchanging the roles of $P$ and $P^{\prime}$ one has $\hat{\varphi}(P)=P^{\prime}$ and $\tilde{\varphi}=\left.\hat{\varphi}\right|_{P}$ is the desired extension.

Corollary 1. Let $M$ and $M^{\prime}$ be two compact, strictly pseudoconvex real hypersurfaces of class $C^{k}(k \geq 2)$ in a complex manifold. Let $C \subset M$ and $C^{\prime} \subset M^{\prime}$ be closed real submanifolds of $M$ and $M^{\prime}$, both of codimension $\geq 2$. Then, every $C R$ isomorphism $M \backslash C \rightarrow M^{\prime} \backslash C^{\prime}$ of class $C^{2}$ extends to a $C R$ isomorphism $M \rightarrow M^{\prime}$ of class $C^{k}$.

Proof. By a result of N. Tanaka (cf. [118]) there is a covariant functor F : CR $\rightarrow$ Cartan from the category $\mathrm{CR}$ of strictly pseudoconvex $\mathrm{CR}$ manifolds, into the category Cartan of Cartan connections, possessing the following properties. On objects, that is, if $M \in \mathrm{Ob}(C R)$, then $F(M)=(P, \omega)$ is a Cartan connection of a certain type $(G, \tilde{G})$ (with $G$ a connected Lie group) such that $P / G=M$, 
and the inclusions $M \backslash C \hookrightarrow M$ and $M^{\prime} \backslash C^{\prime} \hookrightarrow M^{\prime}$ (two morphisms in CR) are compatible with the map on objects $\mathrm{Ob}(C R) \rightarrow \mathrm{Ob}($ Cartan $)$. Therefore, Corollary 1 follows from Theorem 4.

Let $\Omega \subset \mathbb{C}^{n}$ be a smoothly bounded domain with strictly pseudoconvex boundary $\partial \Omega$ and let

$$
\tilde{\Omega}=\left\{(\zeta, z) \in \mathbb{C} \times \Omega:|\zeta|^{2(n+1)} K_{\Omega}(z, z)<1\right\}
$$

be the suspension of $\Omega$. If $n \geq 2$ then $\partial \tilde{\Omega} \subset \mathbb{C}^{n+1}$ is a strictly pseudoconvex real hypersurface of class $C^{n}$. From now on we assume that $n \geq 2$ and consider a biholomorphism $\phi: \Omega \rightarrow \Omega^{\prime}$ onto another smoothly bounded strictly pseudoconvex domain $\Omega^{\prime} \subset \mathbb{C}^{n}$. The remainder of this section is devoted to showing that such $\phi$ extends smoothly up to the boundary. This would give, by following the ideas of I. Naruki (cf. [23]), a new, more geometric, proof to the celebrated Fefferman theorem (cf. [16]). We only look at the case where $\Omega$ is simply connected, yet this assumption may be easily removed (as in [23], p. 121). By the transformation law of Bergman kernels with respect to biholomorphisms

$$
K_{\Omega}(z, z)=\left|J_{\phi}(z)\right|^{2} K_{\Omega^{\prime}}(\phi(z), \phi(z)), \quad z \in \Omega .
$$

As $\Omega$ is assumed to be simply connected there is a holomorphic function $\chi: \Omega \rightarrow \mathbb{C}$ such that $\chi^{n+1}=J_{\phi}$. Next, let us consider the biholomorphism

$$
\begin{gathered}
\hat{\phi}: \mathbb{C} \times \Omega \rightarrow \mathbb{C} \times \Omega^{\prime}, \\
\hat{\phi}(\zeta, z)=(\chi(z) \zeta, \phi(z)), \quad \zeta \in \mathbb{C}, \quad z \in \Omega .
\end{gathered}
$$

Then (by (61) and (62))

$$
\hat{\phi}(\tilde{\Omega})=\widetilde{\Omega^{\prime}} .
$$

Let us apply Corollary 1 to the restriction of $\hat{\phi}$ to $\partial \tilde{\Omega} \cap(\mathbb{C} \times \Omega)$, that is, to the map

$$
\left.\hat{\phi}\right|_{\partial \tilde{\Omega} \cap(\mathbb{C} \times \Omega)}: \partial \tilde{\Omega} \cap(\mathbb{C} \times \Omega) \rightarrow \partial \widetilde{\Omega^{\prime}} \cap\left(\mathbb{C} \times \Omega^{\prime}\right) .
$$

Then (by Corollary 1) the map (63) extends to a $C^{2}$ diffeomorphism of $\partial \tilde{\Omega}$ onto $\partial \widetilde{\Omega^{\prime}}$. This gives a homeomorphism

$$
\Psi: \overline{\tilde{\Omega}} \rightarrow \overline{\widetilde{\Omega^{\prime}}},\left.\quad \Psi\right|_{\tilde{\Omega}}=\left.\hat{\phi}\right|_{\tilde{\Omega}},\left.\quad \Psi\right|_{\partial \tilde{\Omega}} \in C^{2} .
$$

The well known smoothness up to the boundary theorem for the solution to the Dirichlet problem for the Laplacian $\Delta_{0} \equiv \sum_{j=0}^{n} \partial^{2} / \partial z_{j} \partial \bar{z}_{j}$ implies that $\Psi$ is at least $C^{1}$ up to the boundary. As

$$
\Psi(0, z)=\hat{\phi}(0, z)=(0, \phi(z)), \quad z \in \Omega
$$

the restriction of $\Psi$ to $\tilde{\Omega} \cap\left(\{0\} \times \mathbb{C}^{n}\right)$ is a $C^{1}$ extension of $\phi$. The restriction to the boundary of this extension is a $C R$ isomorphism of the boundaries, of class $C^{2}$, and then (again by Corollary 1 applied to $M=\partial \Omega$ and $M^{\prime}=\partial \Omega^{\prime}$ ) of class $C^{\infty}$.

It is an open problem whether Naruki's proof (cf. [23]) may be replicated by using connections in principal bundles (instead of Cartan connections). To any strictly pseudoconvex (in fact, nondegeneracy suffices) $C R$ manifold $M$, one may associate-in the presence of a fixed contact form $\theta$-a canonical linear connection $\nabla$ (the Tanaka-Webter connection of $(M, \theta)$, cf. S.M. Webster [119] and N. Tanaka [120]) and then a connection-distribution in a certain principal bundle $\mathrm{U}(n) \rightarrow P \rightarrow M$. In view of our comments in Section 5 of the present survey, the use of the suspended domain (61) may hide a bond with the theory of weighted Bergman kernels. Additionally, it is an open question whether there is any quantitative relationship between the Fefferman metrics on $\partial \Omega \times S^{1}$ and $\partial \tilde{\Omega} \times S^{1}$. The use of Cauchy completions, as in Naruki's work (cf. op. cit.), is surprisingly similar to the construction of bundle boundaries in the theory of singularities of space-times (cf. B.G. 
Schmidt [24]) and there is certainly a striking similarity between Naruki's canonical metrics of pairs $(P, \omega)$ and the Schmidt metric $g_{S}=\omega \cdot \omega+\eta \cdot \eta$ (say on the total space of the principal bundle $\mathrm{O}(2 n-1,1) \rightarrow O\left(\partial \Omega \times S^{1}, g_{S}\right) \rightarrow \partial \Omega \times S^{1}$ of all Lorentzian frames, where $\omega$ is the Levi-Civita connection 1-form of Fefferman's spacetime $\left(\partial \Omega \times S^{1}, F\right)$ and $\eta$ is the canonical 1-form). There is presently no known analog to Fefferman's theorem for the worm domain $\mathcal{W} \subset \mathbb{C}^{2}$ met in Section 6 of this work (not even for complex analytic automorphisms $\phi \in \operatorname{Hol}(\mathcal{W})$ (the treatment in [121] is known to be incomplete)). It is an open problem whether a Cartan connection argument, as devised by I. Naruki, can be applied to $\partial \mathcal{W} \backslash \mathcal{A}$ (a strictly pseudoconvex CR hypersurface in $\mathbb{C}^{2}$ ), where $\mathcal{A} \subset \partial \mathcal{W}$ is the Levi flat locus of $\partial \mathcal{W}$. As shown by E. Barletta et al. (cf. [122]), timelike lifts of circles on $\partial \mathcal{W}$ (directed towards a point of $\mathcal{A}$ ) run into curvature singularities (of the Fefferman metric on $\left.(\partial \mathcal{W} \backslash \mathcal{A}) \times S^{1}\right)$. It is an open problem whether said curvature singularity may be quantum mechanically resolved (cf. e.g., G.T. Horowitz and D. Marolf [123])—that is, whether the spacial part of the Klein-Gordon operator is essentially self-adjoint as an operator with domain $C_{0}^{\infty}(\Sigma) \subset L^{2}(\Sigma)$, where $\Sigma \subset\left[(\partial \mathcal{W} \backslash \mathcal{A}) \times S^{1}\right]$ is a static slice.

\section{Conclusions}

There are many applications of Fefferman's asymptotic expansion of the Bergman kernel that have not been reviewed here, and that conjecturally admit weighted analogs. Perhaps the first of the sort is P.F. Klembeck's result (cf. [124]) that the holomorphic sectional curvature of (the Bergman metric of) a strictly pseudoconvex domain tends, as the boundary $\partial \Omega$ is approached from inside, to the constant holomorphic sectional curvature of the unit ball. A simpler and logically distinct proof (based on the relationship between the Levi-Civita connection of the Bergman metric, and the Graham-Lee connection of $\Omega$ ) of Klembeck's result, is given by E. Barletta, [125]. E. Barletta's treatment (cf. op. cit.) of Klembeck's theme was inspired by the work of A. Korányi and H.M. Reimann [126]. A. Korányi and H.M. Reimann weakened the hypothesis in Fefferman's theorem by starting with a symplectomorphism $F: \Omega \rightarrow \Omega$ (with respect to the symplectic structure underlying the Kählerian structure of $\Omega$, such as provided by the natural complex structure on $\mathbb{C}^{n}$, and then on $\Omega$, and the Bergman metric on $\Omega$ ) rather than a biholomorphism of $\Omega$. If this is the case, $F$ might fail to extend smoothly to the boundary, but supposing it does, is the boundary values map $f: \partial \Omega \rightarrow \partial \Omega$ at least a contact transformation (rather than a CR isomorphism)? A. Korányi and H.M. Reimann's positive answer to the question exploits the fact that $\varphi(z)=-K(z, z)^{-1 /(n+1)}$ is a defining function for $\Omega$ (where $K(z, \zeta)$ is the unweighted Bergman kernel of $\Omega$ ) and that follows as an elementary application of Fefferman's asymptotic expansion formula. A weighted analog to A. Korányi and H.M. Reimann's result is found by us in [21].

The very discoverer of Bergman kernels (S. Bergman himself [29]) anticipated applications of Bergman kernels, and achieved some (e.g., to the theory of elliptic PDEs, cf. e.g., [127]), reaching beyond complex analysis and, despite G. Fichera's notorious crusade against the use of Bergman kernels (cf. [128,129]), there have been followers such as G.G. Weill [130] and C-Y. Lo [131]. It is an open question whether the kernel function method may be used to study $X$-elliptic equations as appearing in subelliptic theory (cf. e.g., A.E. Kogoj and E. Lanconelli [132], F. Uguzzoni [133]).

Even a review of quantization theory of a modest extension, such as our attempt in Section 4 of the present survey, is bound to notice contributions from both Eastern (e.g., Czechoslovak, Polish, Russian) and Western (e.g., British, Italian) schools of mathematical physics whose interaction with each other is often scarce. Perhaps our gathering of contributors such as A. Odzijewicz, M. Englis, M. Cahen, S. Gutt, and J.H. Rawnsley may provide the reader with some of the unifying means. Followers of M. Cahen et al. (cf. [99]) such as C. Arezzo and A. Loi (cf. [96,97]) succeeded in tying quantization theory to deep results in complex geometry (cf. [98,102]), and deserve further attention. So does the growing literature on Bergman kernels on vector bundles, cf. D. Beltiţă and J.E. Galé [134,135], W. Bertram and J. Hilgert [136], Z. Pasternak-Winiarski, [137]. 
Author Contributions: The authors have equally contributed to the writing of the present survey. All authors have read and agreed to the published version of the manuscript.

Funding: This research received no external funding.

Conflicts of Interest: The authors declare no conflict of interest.

\section{References}

1. Pasternak-Winiarski, Z. On weights which admit the reproducing kernel of Bergman type. Int. J. Math. Math. Sci. 1992, 15, 1-14. [CrossRef]

2. Pasternak-Winiarski, Z. On the dependence of the reproducing kernel on the weight of integration. J. Funct. Anal. 1990, 94, 110-134. [CrossRef]

3. Odzijewicz, A. On reproducing kernels and quantization of states. Commun. Math. Phys. 1988, 114, 577-597. [CrossRef]

4. Horowski, M.; Odzijewicz, A. Geometry of the Kepler system in coherent states approach. Phys. ThéOrique 1993, 59, 69-89.

5. Horowski, M.; Odzijewicz, A. Positive kernels and quantization. J. Geom. Phys. 2013, 63, 80-98.

6. Jakimowicz, G.; Odzijewicz, A. Quantum complex Minkowski space. J. Geom. Phys. 2006, 56, 1576-1599. [CrossRef]

7. Lisiecki, W.; Odzijewicz, A. Twistor flag spaces as phase spaces of conformal particles. Lett. Math. Phys. 1979, 3, 325-334. [CrossRef]

8. Odzijewicz, A. A model of conformal kinematics. Int. J. Theor. Phys. 1976, 15, 575-593. [CrossRef]

9. Odzijewicz, A. A conformal holomorphic field theory. Commun. Math. Phys. 1986, 107, 561-575. [CrossRef]

10. Odzijewicz, A. Coherent states and geometric quantization. Commun. Math. Phys. 1992, 150, $385-413$. [CrossRef]

11. Odzijewicz, A.; Ryzko, A. Coherent states for deformed Jaynes-Cummings model. Rep. Math. Phys. 1997, 40, 277-283. [CrossRef]

12. Odzijewicz, A.; Swietochowski, M. Coherent states map for MIC-Kepler system. J. Math. Phys. 1997, 38, 5010-5030. [CrossRef]

13. Kerzman, N. The Bergman kernel function. Differentiability at the boundary. Math. Ann. 1971, 195, 149-159. [CrossRef]

14. Hörmander, L. $L^{2}$ estimates and existence theorems for the $\bar{\partial}$ operator. Acta Math. 1965, 113, 89-152. [CrossRef]

15. Fefferman, C. Monge-Ampère equations, the Bergman kernel, and geometry of pseudoconvex domains. Ann. Math. 1976, 103, 395-416. [CrossRef]

16. Fefferman, C. The Bergman kernel and biholomorphic mappings of pseudoconvex domains. Invent. Math. 1974, 26, 1-65. [CrossRef]

17. Peloso, M.M. Hankel operators on weighted Bergman spaces on strongly pseudoconvex domains. Ill. J. Math. 1994, 38, 223-249. [CrossRef]

18. Peloso, M.M. Sobolev regularity of the weighted Bergman projections and estimates for minimal solutions to the $\bar{\partial}$-equation. Complex Variables Theory Appl. 1995, 27, 339-363. [CrossRef]

19. Ligocka, E. On the Forelli-Rudin construction and weighted Bergman projections. Stud. Math. 1989, 94, 257-272. [CrossRef]

20. Forelli, F.; Rudin, W. Projections on spaces of holomorphic functions in balls. Indiana Univ. Math. J. 1974, 24, 593-602. [CrossRef]

21. Barletta, E.; Dragomir, S. On boundary behaviour of symplectomorphisms. Kodai Math. J. 1998, 21, $285-305$. [CrossRef]

22. Englis, M. Toeplitz operators and weighted Bergman kernels. J. Funct. Anal. 2008, 255, 1419-1457. [CrossRef]

23. Naruki, I. On extendibility of isomorphisms of Cartan connections and biholomorphic mappings of bounded domains. Tôhoku Math. J. 1976, 28, 117-122. [CrossRef]

24. Schmidt, B.G. A new definition of singular points in general relativity. Gen. Relat. Gravity 1971, 1, $269-280$. [CrossRef]

25. Clarke, C.J.S. The Analysis of Space-Time Singularities; Cambridge University Press: Cambridge, UK, 1993. 
26. Gawdezki, K. Fourier-Like Kernels in Geometric Quantization; Instytut Matematyczny Polskiej Akademi Nauk: Warsaw, Poland, 1976; pp. 5-78.

27. Kostant, B. Quantization and unitary representations. Part I: Prequantization. In Lectures in Modern Analysis and Applications III; Spring: Berlin/Heidelberg, Germany, 2009; pp. 87-206.

28. Barletta, E.; Dragomir, S. On the Djrbashian kernel of a Siegel domain. Stud. Math. 1998, 127, 47-63.

29. Bergman, S. Über die Kernfunktion eines Bereiches und ihr Verhalten am Rande. J. Reine Angew. Math. 1933, $169,1-42$.

30. Aronszajn, N. Theory of reproducing kernels. Trans. Am. Math. Soc. 1950, 68, 337-404. [CrossRef]

31. Zaremba, S. Zarys pierwszych zasad teoryi liczb calkowitych; Akademia Umiejętnści: Kraków, Poland, 1907. (In Polish)

32. Krantz, S.G. A new proof and a generalization of Ramadanov's theorem. Complex Var. Elliptic Equ. 2006, 51, 1125-1128. [CrossRef]

33. Cimmino, G. Nuovo tipo di condizione al contorno e nuovo metodo di trattazione per il problema generalizzato di Dirichlet. Rend. Circ. Matem. Palermo. 1937, LXI, 1-44. [CrossRef]

34. Caccioppoli, R. Sui teoremi d'esistenza di Riemann. Rend. Della Acad. Delle Sci. Fis. Mat. Napoli. 1934, $4,49-54$.

35. Cimmino, G. Opere Scelte; Officine grafiche Francesco Giannini \& Figli: Napoli, Italy, 2002.

36. Djrbashian, M.M.; Karapetyan, A.H. Integral representations for some classes of functions holomorphic in a Siegel domain. J. Math. Anal. Appl. 1993, 179, 91-109. [CrossRef]

37. Burghelea, D.; Hangan, T.; Moscovici, H.; Verona, A. Introducere în Topologia Diferenţială; Editura Ştiinţifică; Scientific Publishing House: Bucure, Romania, 1973.

38. Kohn, J.J. The range of the tangential Cauchy-Riemann operator. Duke Math. J. 1986, 53, 525-545. [CrossRef]

39. Khanh, T.-V. A general method of weights in the $\bar{\partial}-$ Neumann problem. arXiv 2010, arXiv:1001.5093v1.

40. Ramadanov, I. Sur une proprieté de la fonction de Bergman. C. R. Acad. Bulgare Sci. 1967, 20, 759-762.

41. Pasternak-Winiarski, Z.; Wojcieszynski, J. Bergman spaces for holomorphic vector bundles. Demonstr. Math. 1997, 1, 199-214. [CrossRef]

42. Ali, S.T.; Englis, M.; Gazeau, J.P. Vector Coherent States from Plancherel's Theorem, Clifford Algebras and Matrix Domains. J. Phys. A 2004, 37, 6067-6089. [CrossRef]

43. Pasternak-Winiarski, Z.; Wójcicki, M. Weighted generalization of the Ramadanov theorem and further considerations. arXiv 2016, arXiv:1612.05619v1.

44. Dragomir, S.; Tomassini, G. Differential Geometry and Analysis on CR Manifolds; Progress in Mathematics; Birkhäuser: Boston, MA, USA, 2006; Volume 246.

45. Koch, L. Chains on CR manifolds and Lorentz geometry. Trans. Am. Math. Soc. 1988, 307, 827-841. [CrossRef]

46. Jacobowitz, H. Chains in CR geometry. J. Differ. Geom. 1985, 21, 163-191. [CrossRef]

47. Englis, M.; Zhang, G. On a generalized Forelli-Rudin construction. Complex Var. Elliptic Equ. 2006, 51, 277-294. [CrossRef]

48. Roos, G. Weighted Bergman kernels and virtual Bergman kernels. Sci. China Ser. A 2005, 48, $225-237$. [CrossRef]

49. Boas, H.P.; Fu, S.; Straube, E.J. The Bergman kernel function: Explicit formulas and zeros. Proc. Am. Math. Soc. 1999, 127, 805-811. [CrossRef]

50. Huo, Z. The Bergman kernel on some Hartogs domains. arXiv 2015, arXiv:1507.01868v3.

51. Kobayashi, S. Geometry of bounded domains. Trans. Am. Math. Soc. 1959, 92, 267-290. [CrossRef]

52. Libermann, P. Sur les structures presque complexes et autres structures infinitésimales réguliers. Bull. Soc. Math. France 1955, 83, 195-224. [CrossRef]

53. Boothby, W.M. Some fundamental formulas for Hermitian manifolds with vanishing torsion. Am. J. Math. 1954, 76, 509-534. [CrossRef]

54. Vaisman, I. On locally conformal almost Kähler manifolds. Isr. J. Math. 1976, 24, 338-351. [CrossRef]

55. Aubin, T. Variétés hermitiennes localment conformement Kählériennes. C. R. Acad. Sci. Paris 1963, $261,2427-2430$.

56. Kobayashi, S.; Nomizu, K. Foundations of Differential Geometry; Interscience Publishers: New York, NY, USA, 1963.

57. Goldberg, S.I. Curvature and homology; Dover Publications, Inc.: New York, NY, USA, 1962. 
58. Folland, G.B. Harmonic Analysis in Phase Space; Princeton University Press: Princeton, NJ, USA, 1989; Volume 122.

59. Bargmann, V. On a Hilbert space of analytic functions and an associated integral transform. Commun. Pure Appl. Math. 1961, 14, 187-214. [CrossRef]

60. Janson, S.; Peetre, J.; Rochberg, R. Hankel forms and the Fock space. Rev. Mat. Iberoam. 1987, 3, 61-138. [CrossRef]

61. Bommier-Hato, H.; Englis, M.; Youssfi, E.-H. Bergman-type projections in generalized Fock spaces. J. Math. Anal. Appl. 2012, 389, 1086-1104. [CrossRef]

62. Segal, I.E. Mathematical problems of relativistic physics. In Proceedings of the Summer Conference, Boulder, CO, USA, 30 July 1960.

63. Segal, I.E. Mathematical characterization of the physical vacuum for the Bose-Einstein field. Ill. J. Math. 1962, 6, 500-523. [CrossRef]

64. Segal, I.E. The complex-wave representation of the Boson field, in Topics in Functional Analysis. In Advances in Mathematics Supplementary Studies; Gohberg, I., Kac, K., Eds.; Academic Press: Cambridge, MA, USA, 1978; Volume 3.

65. Bargmann, V. Remark on a Hilbert space of analytic functions. Proc. Natl. Acad. Sci. USA 1962, 48, $199-204$. [CrossRef] [PubMed]

66. Bargmann, V. Acknowledgement. Proc. Natl. Acad. Sci. USA 1962, 48, 2204. [CrossRef]

67. Hall, B.C. The Segal-Bargmann "coherent state" transform for compact Lie groups. J. Funct. Anal. 1994, 122, 103-151. [CrossRef]

68. Nelson, E. Analytic vectors. Ann. Math. 1959, 70, 572-615. [CrossRef]

69. van Leeuwen, S. The Segal-Bargmann Transform and Its Generalizations. Ph.D. Thesis, Universiteit Utrecht, Utrecht, The Netherlands, 2009.

70. Barbier, S. The Quantum Mechanical Segal-Bargmann Transform Using Jordan Algebras. Ph.D. Thesis, Universiteit Gent, Gent, Belgium, 2014.

71. Yamamori, A. The Bergman kernel of the Fock-Bargmann-Hartogs domain and the polylogarithm function. Complex Var. Elliptic Equ. 2013, 58, 783-793. [CrossRef]

72. Nakano, S. On complex analytic vector bundles. J. Math. Soc. Jpn. 1955, 7, 1-12. [CrossRef]

73. Mladenov, I.M. MR1194023 (94c:58077) 58F06 81R30 $81 S 10$ Odzijewicz, Anatol (PL-WASWB-IP) Coherent states and geometric quantization. Commun. Math. Phys. 1992, 150, 385-413.

74. Beltiţă, D.; Galé, J.E. Coherent state map quantization in a Hermitian-like setting. J. Geom. Phys. 2015, 92, 100-118. [CrossRef]

75. Coquereaux, R.; Jadczyk, A. Conformal theories, curved phase spaces, relativistic wavelets and the geometry of complex domains. Rev. Math. Phys. 1990, 2, 1-44. [CrossRef]

76. Englis, M. Weighted Bergman kernels and quantization. Commun. Math. Phys. 2002, 227, 211-241. [CrossRef]

77. Kisil, V.V. Integral representations and coherent states. Bull. Belg. Math. Soc. Simon Stevin 1995, 2, 529-540. [CrossRef]

78. Monastyrskii, M.I.; Perelomov, A.M. Coherent states and bounded homogeneous domains. Dokl. Akad. Nauk SSSR 1972, 207, 1303-1305. (In Russian)

79. Dragomir, S.; Masamune, M. Cauchy-Riemann orbifolds. Tsukuba J. Math. 2002, 26, 351-386. [CrossRef]

80. Barletta, E.; Dragomir, S.; Duggal, K.L. Foliations in Cauchy-Riemann Geometry; Mathematical Surveys and Monographs; American Mathematical Society: Providence, RI, USA, 2007; Volume 140.

81. Balasubramanian, V.; Hassan, S.F.; Keski-Vakkuri, E.; Naqvi, A. A Space-Time Orbifold: A Toy Model for a Cosmological Singularity. arXiv 2002, arXiv:hep-th/0202187v2.

82. Behrndt, K.; Lust, D. Branes, waves, and AdS orbifolds. JHEP 1999. [CrossRef]

83. Son, J. String Theory on $\mathrm{AdS}_{3} / \mathbb{Z}_{N}$. arXiv 2012, arXiv:hep-th/0107131.

84. Martinec, E.J.; McElgin, W. String theory on AdS orbifolds. JHEP 2002. [CrossRef]

85. Satake, I. The Gauss-Bonnet theorem for $V$-manifolds. J. Math. Soc. Jpn. 1957, 9, 464-492. [CrossRef]

86. Takeuchi, Y. Orbi-maps and 3-orbifolds. Proc. Jpn. Acad. Ser. A 1989, 65, 345-347. [CrossRef]

87. Yamasaki, M. Maps between orbifolds. Proc. Am. Math. Soc. 1990, 109, 223-232. [CrossRef]

88. Moerduk, I.; Pronk, D.A. Orbifolds, Sheaves and Grupoids. K-Theory 1997, 12, 3-21. [CrossRef]

89. Chen, W.M. On a notion of maps between orbifolds II: homotopy and CW complex. arXiv 2006, arXiv:math/0610085v1. 
90. Hurt, N. Geometric Quantization in Action; Reidel: Dordrecht, The Netherlands, 1983.

91. Simms, D.J. Geometric quantization of energy levels in the Kepler problem. J. Geom. Phys. 1985, 2, 17-24.

92. Aazami, A.B. ; Petters, A.O.; Rabin, J.M. Orbifolds, the A, D, E family of caustic singularities, and gravitational lensing. arXiv 2010, arXiv:1004.0516v1.

93. Aazami, A.B.; Keeton, C.R.; Petters, A.O. Lensing by Kerr black holes. II: Analytical study of quasi-equatorial lensing observables. arXiv 2011, arXiv:1102.4304v2.

94. Petters, A.O.; Werner, M.C. Mathematics of gravitational lensing: multiple imaging and magnification. arXiv 2009, arXiv:0912.0490v1.

95. Loi, A. Quantization of bounded domains. J. Geom. Phys. 1999, 29, 1-4. [CrossRef]

96. Arezzo, C.; Loi, A. Quantization of Kähler manifolds and the asymptotic expansion of Tian-Yau-Zelditch. J. Geom. Phys. 2003, 47, 87-99. [CrossRef]

97. Arezzo, C.; Loi, A. Moment maps, scalar curvature and quantization of Kähler manifolds. Commun. Math. Phys. 2004, 246, 543-559 [CrossRef]

98. Tian, G. On a set of polarized Kähler metrics on algebraic manifolds. J. Differ. Geom. 1990, 32, 99-130. [CrossRef]

99. Cahen, M.; Gutt, S.; Rawnsley, J.H. Quantization of Kähler manifolds I: Geometric interpretation of Berezin's quantization. J. Geom. Phys. 1990, 7, 45-62.

100. Berezin, F.A. Quantization. Math. USSR Izv. 1974, 8, 1109-1165. (In Russian) [CrossRef]

101. Wightman, A.S. MR0395610 (52 \# 16404) 81.5881.46 Berezin, F. A. [Berezin, Feliks Aleksandrovich] Quantization. Izv. Akad. Nauk SSSR Ser. Mat. 1974, 38, 1116-1175.

102. Zelditch, S. Szegö Kernel and a theorem of Tian. J. Differ. Geom. 1990, 32, 99-130.

103. Lu, Z. On the lower terms of the asymptotic expansion of Tian-Yau-Zelditch. Am. J. Math. 2000, 122, $235-273$. [CrossRef]

104. Coifman, R.R.; Rochberg, R. Representation theorems for holomorphic and harmonic functions in $L^{p}$. Astérisque 1980, 77, 11-66.

105. Saitoh, S. Hilbert spaces induced by Hilbert space valued functions. Proc. Am. Math. Soc. 1983, 89, 74-78. [CrossRef]

106. Saitoh, S. One approach to some general integral transforms and its applications. Integral Transform. Spec. Funct. 1995, 3, 49-84. [CrossRef]

107. Barletta, E.; Dragomir, S. Uniform approximation of holomorphic forms. Complex Var. 1998, 35, $359-366$. [CrossRef]

108. Faber, V.; Mycielski, J. Applications of learning theorems. Fund. Inform. 1991, 15, 145-167.

109. Mycielski, J.; Świerczkowski, S. Uniform approximation with linear combinations of reproducing kernels. Stud. Math. 1996, 121, 105-114.

110. Englis, M. Analytic continuation of weighted Bergman kernels. J. Math. Pures Appl. 2010, 94, $622-650$. [CrossRef]

111. Rudin, W. Functional Analysis. In International Series in Pure and Applied Mathematics, 2nd ed.; McGraw-Hill Inc.: New York, NY, USA, 1991.

112. Englis, M. Weighted Bergman kernels for logarithmic weights. Pure Appl. Math. Q. 2010, 6, $781-813$. [CrossRef]

113. Krantz, S.G. Function Theory of Several Complex Variables; Wiley: New York, NY, USA, 1982.

114. D'Angelo, J.P. An explicit computation of the Bergman kernel function. J. Geom. Anal. 1994, 4, $23-34$. [CrossRef]

115. Francsics, G.; Hanges, N. The Bergman kernel of complex ovals and multivariable hypergeometric functions. J. Funct. Anal. 1996, 142, 494-510. [CrossRef]

116. Beberok, T. An explicit computation of the Bergman kernel function. Complex Var. Elliptic Equ. 2015, 60, 1058-1067. [CrossRef]

117. Helgason, S. Differential Geometry, Lie groups, and Symmetric Spaces; Academic Press: New York, NY, USA; London, UK, 1978.

118. Tanaka, N. On generalized graded Lie algebras and geometric structures I. J. Math. Soc. Jpn. 1967, 19, $215-254$. [CrossRef]

119. Webster, S.M. Pseudohermitian structures on a real hypersurface. J. Differ. Geom. 1978, 13, 25-41. [CrossRef] 
120. Tanaka, N. A Differential Geometric Study on Strongly Pseudo-Convex Manifolds; Kinokuniya Book Store: Tokyo, Japan, 1975.

121. Chen, S.-C.Characterization of the automorphisms on the Barrett and the Diederich-Fornaess worm domains. Trans. Am. Math. Soc. 1993, 338, 431-440.

122. Barletta, E.; Dragomir, S.; Peloso, M.M. Worm domains and Fefferman space-time singularities. J. Geom. Phys. 2017, 120, 142-168. [CrossRef]

123. Horowitz, G.T.; Marolf, D. Quantum probes of spacetime singularities. arXiv 1995, arXiv:gr-qc/9504028v3.

124. Klembeck, P.F. Kähler metrics of negative curvature, the Bergmann metric near the boundary, and the Kobayashi metric on smooth bounded strictly pseudoconvex sets. Indiana Univ. Math. J. 1978, 27, 275-282. [CrossRef]

125. Barletta, E. On the boundary behaviour of the holomorphic sectional curvature of the Bergman metric. Matematiche 2006, 61, 301-316.

126. Korányi, A.; Reimann, H.M. Contact transformations as limits of symplectomorphisms. Mathematics 1994, 318, 1119-1124.

127. Bergman, S.; Schiffer, M. Kernel Functions and Elliptic Differential Equations in Mathematical Physics; Dover Publications, Inc.: Mineola, NY, USA, 1953.

128. Fichera, G. Sui teoremi d'esistenza della teoria del potenziale e della rappresentazione conforme. II. Atti Accad. Naz. Lincei Rend. Cl. Sci. Fis. Mat. Nat. 1951, 10, 452-457. (In Italian)

129. Fichera, G. Sulla "Kernel function". Boll. Un. Mat. Ital. 1952, 7, 4-15. (In Italian)

130. Weill, G.G. On Bergman's kernel function for some uniformly elliptic partial differential equations. Proc. Am. Math. Soc. 1965, 16, 1299-1304.

131. Lo, C.-Y. Bergman's kernel function on a class of elliptic partial differential equations in four variables. Ann. Mat. Pura Appl. 1968, 79, 93-105.

132. Kogoj, A.E.; Lanconelli, E. Liouville theorem for X-elliptic operators. Nonlinear Anal. 2009, 70, $2974-2985$. [CrossRef]

133. Uguzzoni, F. Estimates for the Green function for X-elliptic operators. Math. Ann. 2015, 361, 169-190. [CrossRef]

134. Beltiţă, D.; Galé, J.E. Reproducing kernels and positivity of vector bundles in infinite dimensions. arXiv 2014, arXiv:1402.0458v1.

135. Beltiţă, D.; Galé, J.E. Linear connections for reproducing kernels on vector bundles. arXiv 2013, arXiv:1206.3969v3.

136. Bertram, W.; Hilgert, J. Reproducing kernels on vector bundles. arXiv 2020, arXiv:1402.0458.

137. Pasternak-Winiarski, Z. Reproducing kernels for holomorphic vector bundles. Adv. Math. Sci. J. 2016, $5,25-32$.

(C) 2020 by the authors. Licensee MDPI, Basel, Switzerland. This article is an open access article distributed under the terms and conditions of the Creative Commons Attribution (CC BY) license (http://creativecommons.org/licenses/by/4.0/). 